\title{
Power amplification via compliant layer interdigitation and dielectrophoretic structuring of PZT particle composites
}

\author{
By \\ Zachary Pessia \\ B.S., Olivet Nazarene University, 2017 \\ Submitted to the graduate degree program in Mechanical Engineering and the Graduate Faculty \\ of the University of Kansas in partial fulfillment of the requirements \\ for the degree of Master of Science. \\ Chair: Dr. Elizabeth Friis \\ Dr. Kenneth Fischer \\ Dr. Ronald Barrett
}

Date Defended: 23 August 2019 
The thesis committee for Zachary Pessia certifies that this is the approved version of the following thesis:

\section{Power amplification via compliant layer interdigitation and dielectrophoretic structuring of PZT particle composites}

Chair: Dr. Elizabeth Friis

Date Approved: 28 August 2019 


\section{Abstract}

Nonunion occurs in up to $10 \%$ of all fractures, with about $8 \%$ of all femoral fractures ending in nonunion, or failed healing with current fixation methods ${ }^{1,2}$. These failure rates can be caused by factors such as diabetes, osteoporosis, tobacco use, and severe tissue damage $\mathrm{e}^{3,4}$. According to the FDA, it takes a minimum of nine moths to declare nonunion after trauma, with no progress in healing for three months ${ }^{5,6}$. Some adjunct therapy methods are being used to combat these failure rates such as the OsteoGen ${ }^{\mathrm{TM}}$ direct current bone growth stimulator. However, these devices require an implanted battery that will eventually need removed.

The evolution of portable electronics has led to recent popularity of piezoelectric materials for energy harvesting, especially for devices deployed remotely or in vivo. Intramedullary nails could utilize the energy harvesting capabilities of piezoelectric materials to provide electrical stimulation at the fracture site without an implanted battery. However, the efficiency of piezoelectric generators harvesting energy from the human body is lacking due to off-resonance loading $^{7}$. In addition, piezoelectric ceramics are expensive to manufacture, dense, brittle, and difficult to use in high strain environments.

Piezoelectric composites composed of ferroelectric particles distributed in a polymer matrix are desirable due to low cost and tunable properties. In this study, Compliant Layer Adaptive Composite Stacks (CLACS) made with thin piezoelectric composite layers structured by dielectrophoresis (DEP) were investigated to increase the energy harvesting efficiency at low frequencies. To predict power generation capabilities, a theoretical model was developed by using established particle composite models in conjunction with a shear lag structural mechanics model for CLACS. Granular composite discs of lead zirconate titanate particles in an epoxy matrix were manufactured at a 50\% volume fraction and structured by DEP, if applicable. 
CLACS were manufactured using ten composite discs and two compliant layer thicknesses. The stacks were electromechanically tested by varying load, frequency, and resistance. Experimental results showed an increase in power amplification with DEP structured discs and compliant layers. In addition, the theoretical model accurately predicts power production for both $0-3$ and 1-3 CLACS at low frequencies. DEP structured particle composite CLACS can provide a method of energy harvesting for devices in remote locations, especially in low frequency high strain environments.

Future work could continue the development of piezoelectric particle composite CLACS for use in intramedullary nails. Such studies would evaluate the performance of ring shaped piezoelectric composites, develop theoretical understanding for ring shaped CLACS, investigate fatigue strength of piezoelectric particle composites, and evaluate impact strength of particle composite CLACS as compared to ceramic CLACS. Lastly, overall improvements to particle composite manufacturing methods to reduce variability could be investigated. 


\section{Acknowledgements}

- Dr. Elizabeth Friis for all of her guidance and support throughout my graduate career. Her amazing ideas and wisdom are what ignite the innovation and translation to real products.

\section{- Craig Cunningham, Ember Krech, Nolan Norton, Morghan Alters, Colton Lowe, and}

Eileen Cadel for all of their support, help in manufacturing and brainstorming, and friendship.

- Craig Cunningham for all of his help from the beginning with helping manufacture my specimen, coming up with novel solutions for the IM nail design, imparting experience and wisdom with machining and design, reading articles, and dealing with my optimism and positivity amidst complete failure.

○ Ember Krech for imparting all of her wisdom and experience in lab skills, biomechanics, study design, manufacturing methods, testing, statistics, brainstorming and encouragement. In addition, I would like to thank Ember for being an example of an honorable graduate researcher and $\mathrm{PhD}$ candidate.

- Nolan Norton for all of the encouragement and friendship throughout my graduate career. I'd also like to thank him for all of the good conversations, guidance, wisdom from experience, and for Miles.

- Morghan Alters for all of her help with utilizing ultrasound to verify and test my specimen, and for providing snacks and understanding the sweet tooth craving.

- Colton Lowe for imparting his knowledge on biomechanics, statistics, specimen manufacturing, and testing.

- Eileen Cadel for welcoming me to the lab and imparting knowledge and wisdom in testing, manufacturing, reading and acquiring articles, and biomechanics. 
- Dr. Kenneth Fischer for agreeing to be a part of my thesis committee and helping with my thesis project and for imparting your knowledge and wisdom in class.

- Dr. Robert Barrett for agreeing to be a part of my thesis committee and providing wisdom and expertise with piezoelectric materials and theory.

- The Mechanical Engineering Department for providing the funding through teaching assistantships and implementing a successful graduate program.

- Ansel Armstrong and William Vincent for always answering questions and helping me no matter the issue or concern. I would also like to thank them for always being so welcoming and nice.

- All of my lab mates, Camilo Giraldo, Ryan Wagner, Wyatt Hursh, Chris Bowen, and Kait Howard for all of your friendships, help, and support throughout my graduate school career. You all make graduate school enjoyable and have all helped me complete this work in one way or another.

- My parents Wayne and Anne Pessia and siblings Jacob and Katelyn Pessia for always encouraging me from afar and supporting me. You gave me the drive and passion for seeking a career that can help people and were always there to listen and encourage me.

- Ashley Pessia for being the joy of my heart and encouraging and supporting me always. It almost seemed impossible to plan a wedding, get married, and both finish our graduate careers, but we did it. 


\section{Table of Contents}

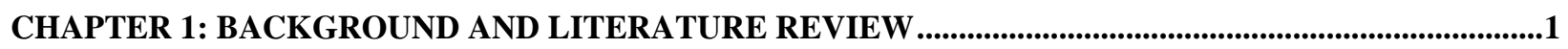

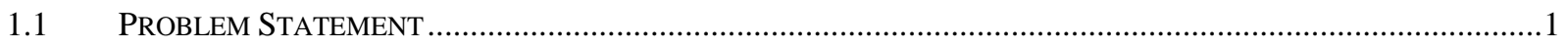

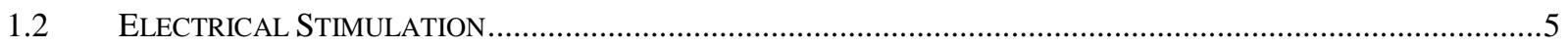

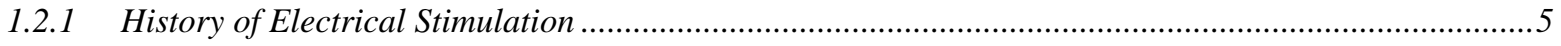

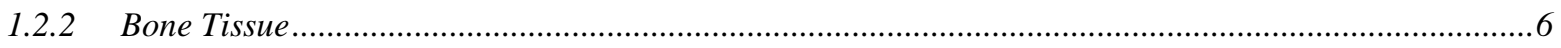

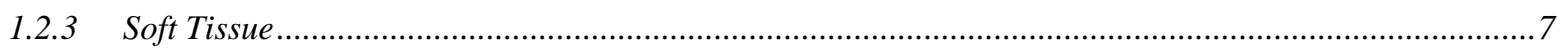

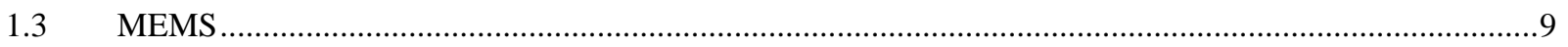

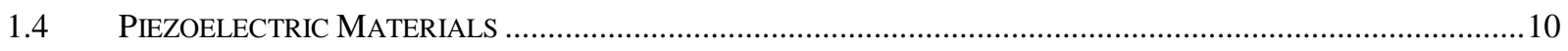

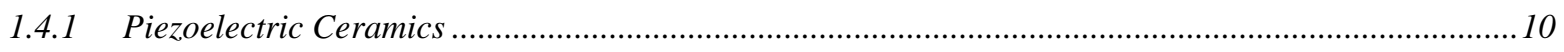

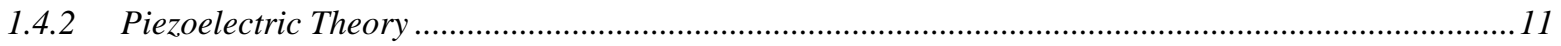

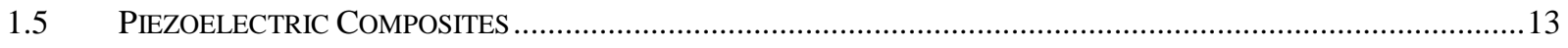

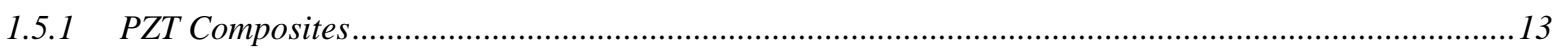

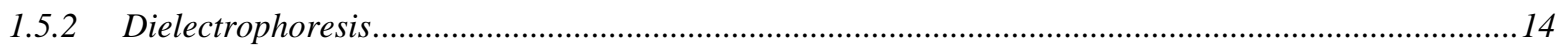

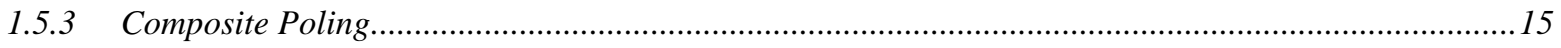

CHAPTER 2: SMART MATERIALS AND STRUCTURES PAPER .............................................................17

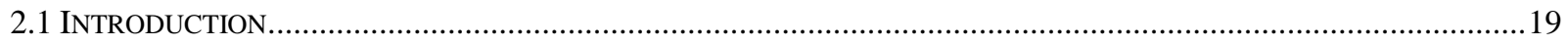

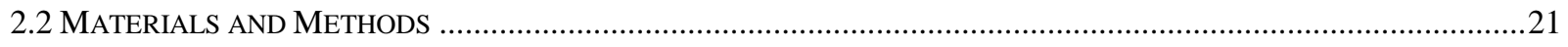

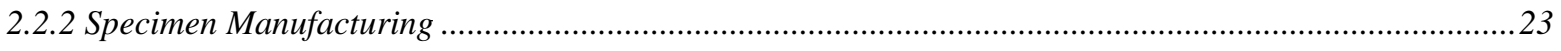

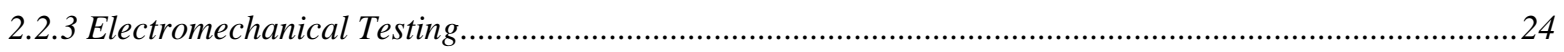

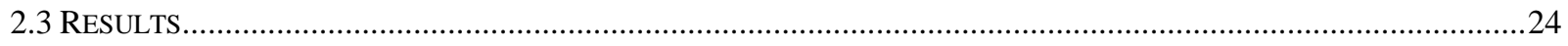

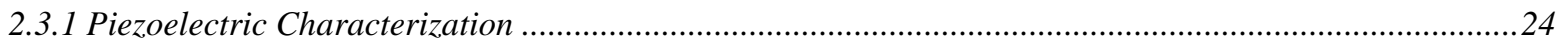

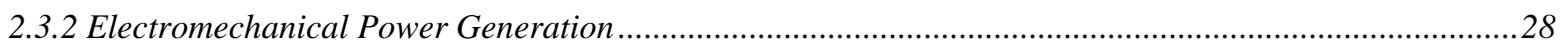

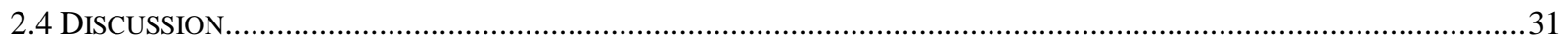

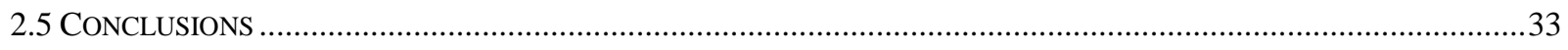

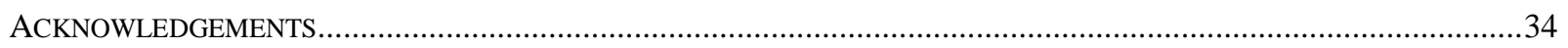




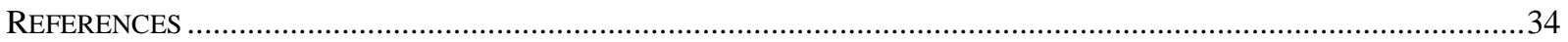

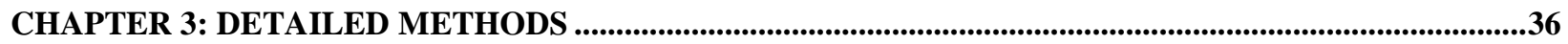

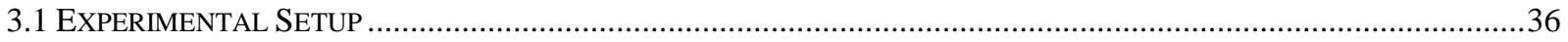

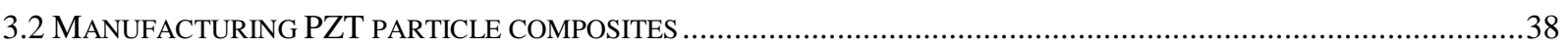

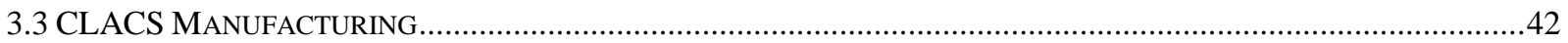

3.4 DEVELOPING THE THEORETICAL MODEL ……….........................................................................................

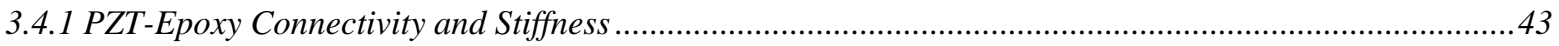

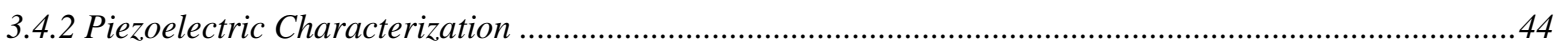

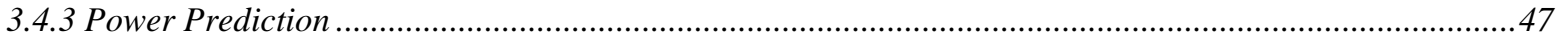

CHAPTER 4 : CONCLUSIONS AND FUTURE WORK .........................................................................................50

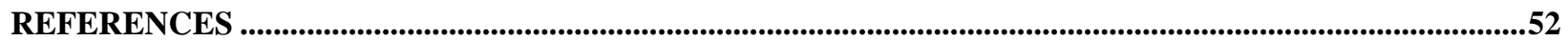

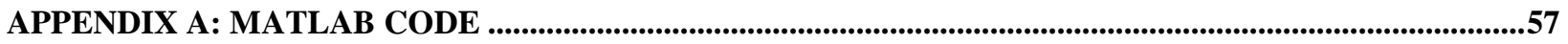

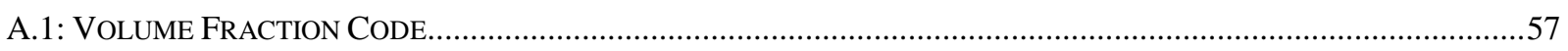

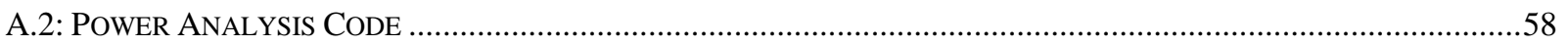

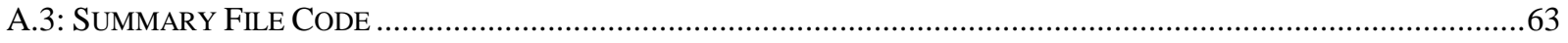

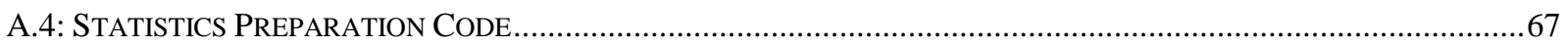

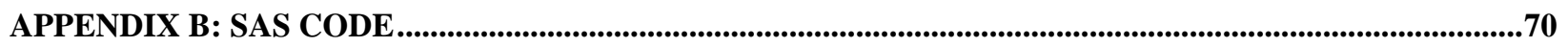

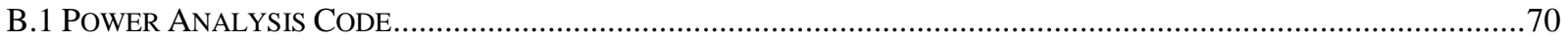

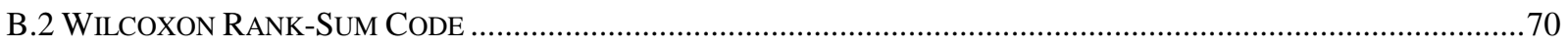

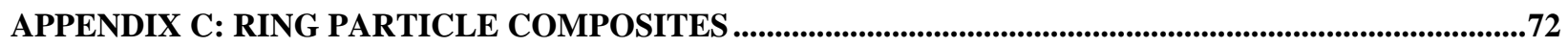

C.1: FRACTURE FIXATION IMPLEMENTATION...........................................................................................

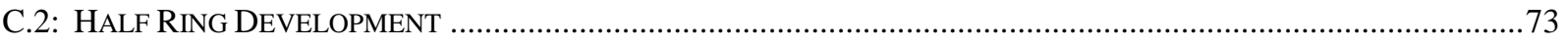

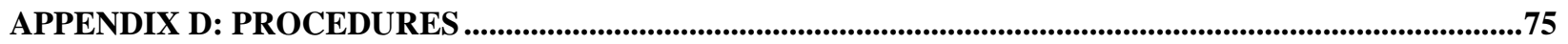

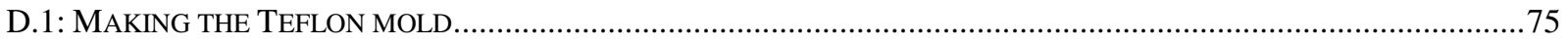

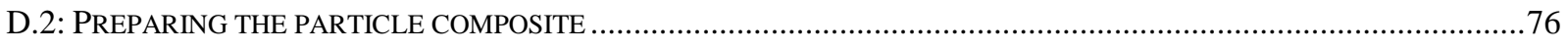




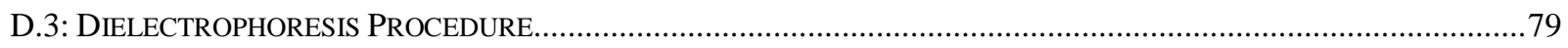

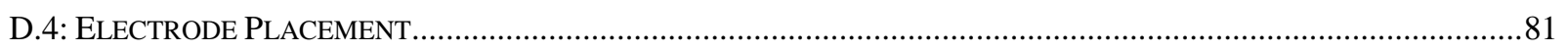

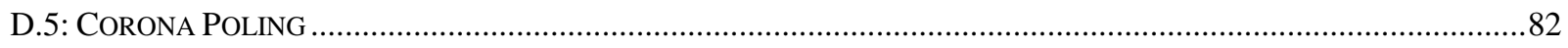

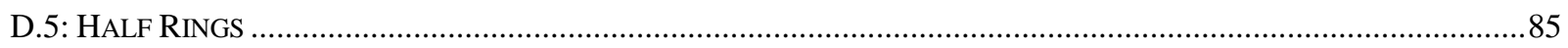

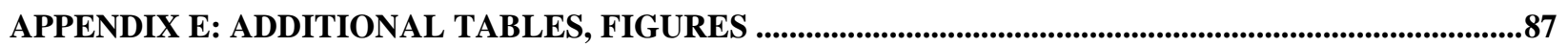

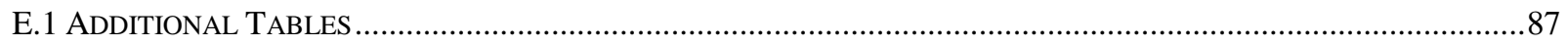

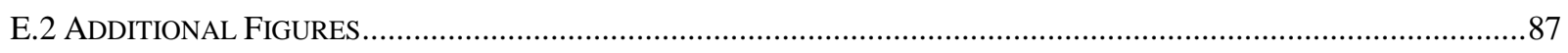

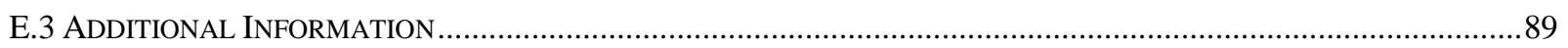




\section{List of Figures}

Figure 1.1: PZT Fiber composite stack before encapsulation (Left) and after encapsulation

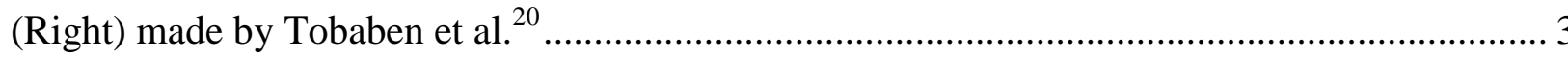

Figure 1.2: Compliant layer adaptive composite stack (CLACS) ....................................... 4

Figure 1.3: The perovskite structure of Lead Zirconate Titanate (PZT), showing the structure may be polarized when below the Curie temperature (TC). (Public domain, commons.wikipedia.org, Labeled for Reuse).

Figure 1.4: The poling treatment for PZT showing the random alignment of domains (left) and the resulting net polarity and anisotropy (right).

Figure 1.5: Dielectrophoretic structuring of particles showing the randomly dispersed particles (left) and the aligning of particles under an alternating electric field (right). 15

Figure 1.6: Diagrams for setup of both the parallel plate-contact method (A) and the corona discharge poling method (B). (C IOP Publishing. Reproduced with permission. All rights reserved 16

Figure 2.1: Schematic of mold setup for dielectrophoresis processing 22

Figure 2.2: Maximum power of theoretical models for structured and random CLACS and the experimental results for average maximum power.

Figure 2.3: Electromechanical coupling factor $(\mathrm{k} 33)$ of theoretical models and experimental results for both random and structured CLACS.

Figure 2.4: Average power output as a function of structuring type, compliant layer thickness, and resistance load of a 50\% VF composite stack when mechanically loaded at $1000 \mathrm{~N}$ and $2 \mathrm{~Hz}$. 
Figure 2.5: Average voltage with respect to structuring type, compliant layer thickness, and testing frequency for $50 \%$ VF composite CLACS. Representative voltage data presented at maximum power $(1000 \mathrm{~N}, 40.5 \mathrm{M} \Omega)$. 36

Figure 3.1: Entire experimental manufacturing setup for poling (left) and dielectrophoresis

(right). 36

Figure 3.2: Schematic of setup of both the parallel plate-contact method (A) and the corona discharge poling method (B). (C IOP Publishing. Reproduced with permission. All rights reserved. 37

Figure 3.3: Schematic of mold setup for dielectrophoresis processing. 37

Figure 3.4: Teflon mold and electrodes for dielectrophoresis. 39

Figure 3.5: Setup for 30 discs to be sprayed for electrode application 40

Figure 3.6: Corona discharge setup with 10 needle points connected in parallel with the aluminum plate as ground. 41

Figure 3.7: Piezoelectric particle stack without a compliant layer $(11 \mathrm{~mm}$ x $11 \mathrm{~mm}$ x $8 \mathrm{~mm}) \ldots . .47$ Figure 3.8: Max power (left) and $\mathrm{k}_{33}$ (right) theoretical predictions vs. experimental results under mechanical loading $(1000 \mathrm{~N}, 5 \mathrm{~Hz}, 5 \mathrm{M} \Omega$. 49

Figure A.1: Original modular IM nail 72

Figure A.2: Epoxy encapsulation support concept for half rings or rings ............................. 73

Figure A.3: Half ring stacking concept with epoxy support shown in Figure 19..................... 73

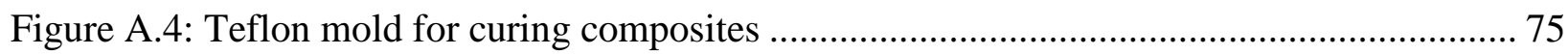

Figure A.5: Crucible with particle powder for sintering ................................................... 76 
Figure A.6: Delrin plates with electrodes and Teflon mold used for curing composites and performing DEP. 77

Figure A.7: Oscilloscope (top) and function generator (bottom). 79

Figure A.8: HV amplifier input form function generator and output for monitoring sine wave.. 80 Figure A.9: Reusable Teflon mat used to apply conductive spray 81

Figure A.10: Entire corona poling setup with 10 corona needles and a voltmeter to monitor voltage input 82

Figure A.11: Corona poling setup for through thickness poling of 10 composite discs. 83

Figure A.12: Half-ring negative (SolidWorks) 3D-printed for silicone mode. 85 Figure A.13: Maximum power produced vs. frequency at $500 \mathrm{~N}$ and at the resistance for maximum power at each frequency $(167 \mathrm{M} \Omega, 77 \mathrm{M} \Omega, 57 \mathrm{M} \Omega, 40.5 \mathrm{M} \Omega$ respectively). 87

Figure A.14: Maximim power produced vs. frequency at $1000 \mathrm{~N}$ and at the resistance for maximum power at each frequency $(167 \mathrm{M} \Omega, 77 \mathrm{M} \Omega, 57 \mathrm{M} \Omega, 40.5 \mathrm{M} \Omega$ respectively).... 88

Figure A.15: Venn diagram to capture the development of the theoretical model. 88

\section{List of Tables}

Table 3.1: Average peak power with respect to thickness and structuring type $(1000 \mathrm{~N}, 5 \mathrm{~Hz}$,

$40.5 \mathrm{M} \Omega$ 30

Table A.1: Average maximum power for all frequencies. 87 


\section{List of Variables}

$\begin{array}{llr}E & \text { Young's modulus } & (\mathrm{N} / \mathrm{m} 2) \\ \zeta & \text { measure of reinforced geometry } & \sim \\ \mathrm{M} & \text { depolarization factor } & \sim \\ K & \text { relative dielectric constant } & \sim \\ \mathrm{E} & \text { permittivity } & (\mathrm{farad} / \mathrm{m}) \\ \mathrm{H} & \text { inverse of depolarization factor } & \sim \\ \Phi & \text { volume fraction of PZT phase } & \sim \\ d_{33} & \text { piezoelectric charge constant } & (\mathrm{C} / \mathrm{N}) \\ R & \text { average particle size / interpartcle dist. } \\ k_{33} & \text { electromechanical coupling factor } & \sim \\ S & \text { elastic compliance } & \sim \\ \text { W } & \text { work done to composite } & (\mathrm{m} 2 / \mathrm{N}) \\ \mathrm{F} & \text { frequency } & (\mathrm{J})\end{array}$

\section{List of Equations}

Equation 2.1: Yamda model for dielectric constant of 0-3 composites ........................................ 24

Equation 2.2: Yamada model for piezoelectric charge constant of 0-3 composites ..................... 25

Equation 2.3: Bowen model for dielectric constant of quasi 1-3 composites............................... 25

Equation 2.4: Van den Ende model for piezoelectric charge constant of 1-3 composites............. 25

Equation 2.5: Theoretical Baseline Power.................................................................................. 26

Equation 2.6: Halpin-Tsai model for particle composite properties............................................. 26

Equation 2.7: Krech model for shear lag theory compliant layer amplification........................... 26

Equation 2.8: Modified Krech model to achieve theoretical $\mathrm{k}_{33}$................................................ 27

Equation 3.1: Halpin-Tsai model for two phase particle composites .......................................... 43

Equation 3.2: Halpin-Tsai $\mathrm{N}$ factor to relate shape and modulus ratios ........................................ 44 
Equation 3.3: Halpin-Tsai $\zeta$ factor to describe effect of geometry.

Equation 3.4: Halpin-Tsai model for continuous aligned fibers in a polymer matrix .................. 44

Equation 3.5: Polarization factor of a ferroelectric ceramic particle ............................................ 44

Equation 3.6: Shape factor for defining electic potentials produced ............................................ 44

Equation 3.7: Yamda model for dielectric constant of 0-3 composites ........................................ 45

Equation 3.8: Yamada model for piezoelectric charge constant of 0-3 composites ..................... 45

Equation 3.9: Bowen model for dielectric constant of quasi 1-3 composites............................... 45

Equation 3.10: Van den Ende model for piezoelectric charge constant of 1-3 composites........... 46

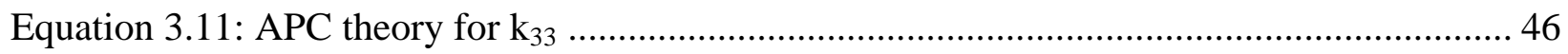

Equation 3.12: Elastic compliance of composite ........................................................................... 46

Equation 3.13: Theoretical permittivity of the composite ………............................................. 47

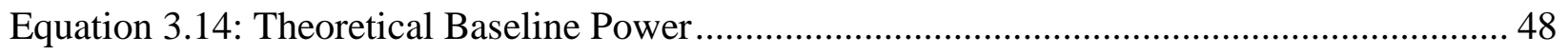

Equation 3.15: Krech model for shear lag theory compliant layer amplification ......................... 48

Equation 3.16: Modified Krech model to achieve theoretical $\mathrm{k}_{33}$............................................. 48 


\section{Chapter 1: Background and Literature Review}

This chapter discusses the need for this work followed by background information and a review of literature to provide better understanding. Knowledge pertaining to piezoelectric materials, piezoelectric theory, electromechanical properties of tissues, and electrical stimulation is necessary to better understand this work. The current needs pertaining to fracture fixation will be introduced, along with history and understanding of electrical stimulation. Piezoelectric composite design and the use of electrical stimulation in fracture fixation applications will be explored.

\subsection{Problem Statement}

There are many levels of impaired fracture healing resulting in pain, functional disability, lack of healing, and the need for surgical intervention ${ }^{5,8}$. As healing time progresses, a fracture is considered a delayed union. This continues until surgical intervention is required and the fracture is labeled a nonunion ${ }^{5,6}$. Nonunion rates for all fractures are between $1.9 \%$ and $10 \%$, with 100,000 of fractures going to nonunion each year in the United States ${ }^{1}$. For long bones specifically, femoral nonunions occur in about $8 \%$ in modern antegrade nailing, and tibial nonunions occur in $4.6 \%$ of tibial nailing for fixation of fractures ${ }^{2}$. These failure rates are caused by numerous factors; however, it is known that factors including diabetes, osteoporosis, tobacco use, alcohol, and drugs can increase risk of nonunion and failed fusion ${ }^{3,4}$ In addition, biological factors such as severe tissue damage, poor blood supply, or infections are also responsible for major disturbances in fracture healing ${ }^{6,9}$. For cases of nonunion, the Food and Drug Administration states a nonunion is established after a minimum of nine months after trauma with no progress toward healing for three months ${ }^{5,8}$. Along with pain and loss of function caused 
by nonunions and fixation failure, there are tremendous costs incurred. There are both direct and indirect costs associated with nonunion complications. According to Antonova et el., the median total cost of successful union is $\$ 11,686$ compared to $\$ 25,556$ for tibial shaft nonunions ${ }^{10}$. Therefore, nonunion can result in extended time in pain and loss of function along with more than doubling the costs of healing.

Ever since the discovery of piezoelectric potentials of bone by Fukada and Yasuda ${ }^{11}$, direct current (DC) stimulators have been used clinically to improve bone healing and treat nonunions ${ }^{12,13}$. However, current DC stimulators require a battery that is invasively implanted along with fracture fixation devices ${ }^{12}$. This results in a second surgery for removal of the battery, incurring additional cost and discomfort. There are methods of providing electrical stimulation externally, such as capacitive coupling (CC) and pulsed electromagnetic fields (PEMF), but DC stimulators have the advantage of stimulating the bone directly at the fracture site ${ }^{14}$. Recent medical applications have proposed the use of piezoelectric materials to promote bone growth without a battery ${ }^{15}$. The mechanical stresses on piezoelectric materials distort an internal diploe moment and generate electrical potentials in the loading direction ${ }^{7}$. Medical devices can utilize these piezoelectric generators to deliver electric current to specific areas of influence around the implant $^{7}$. Self-powered fracture fixation devices can generate electrical energy from cyclic mechanical loading in the femur during walking and provide DC stimulation without a battery.

Piezoelectric ceramics are popular and have excellent piezoelectric and dielectric properties; however, ceramics are brittle, non-ductile and difficult to shape. This is a limiting factor for high impact or high strain energy harvesting ${ }^{16,17}$. To combat these issues, lead zirconium titanate (PZT) ceramics are combined with a low density, high flexibility polymer ${ }^{16}$. In a previous study, piezoelectric spinal interbody implants were made by stacking PZT fiber composite layers 
(Figure 1.1). Each layer was made by aligning PZT fibers in an epoxy matrix in the thickness direction and slicing the composite to the desired thickness with a diamond blade saw ${ }^{18}$. The implants were tested in a pilot ovine study to stimulate the fusion site with DC current, and

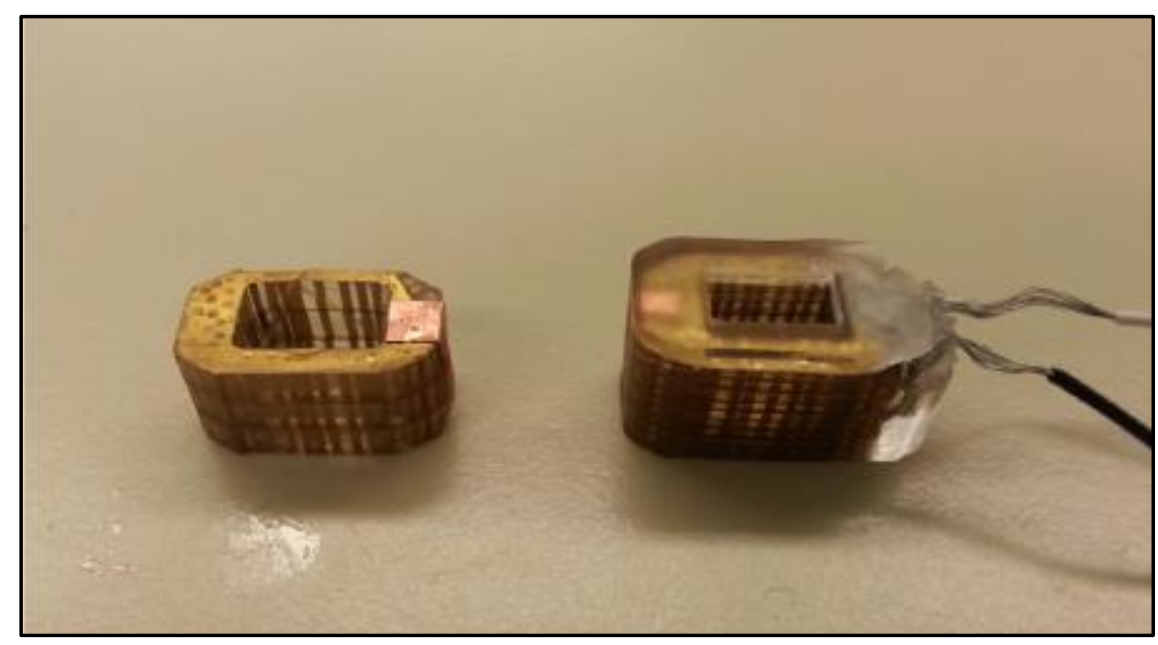

Figure 1.1: PZT Fiber composite stack before encapsulation (Left) and after encapsulation (Right) made by Tobaben et al. ${ }^{20}$

fusion was dramatically enhanced after just 6 weeks when compared to a control ${ }^{18}$. However, the fabrication process is very labor intensive, expensive, and contains a large number of production steps with brittle and fragile ceramic fibers not scalable for use in medical devices ${ }^{19,20}$.

Composites made of thin PZT layers stacked with compliant layers of epoxy adhered between each PZT layer were studied in order to improve manufacturability. These composites are called Compliant Layer Adaptive Composite Stacks (CLACS) and generate sufficient power while achieving desired compliance due to the epoxy layer (Figure 1.2$)^{19}$. While the original purpose for CLACS was to simplify manufacturing, the addition of a compliant layer between PZT layers significantly increased power production capabilities ${ }^{19}$. However, the application of such composites is restricted due to cost of custom designed piezoelectric materials and the brittle nature of piezoelectric ceramics. 


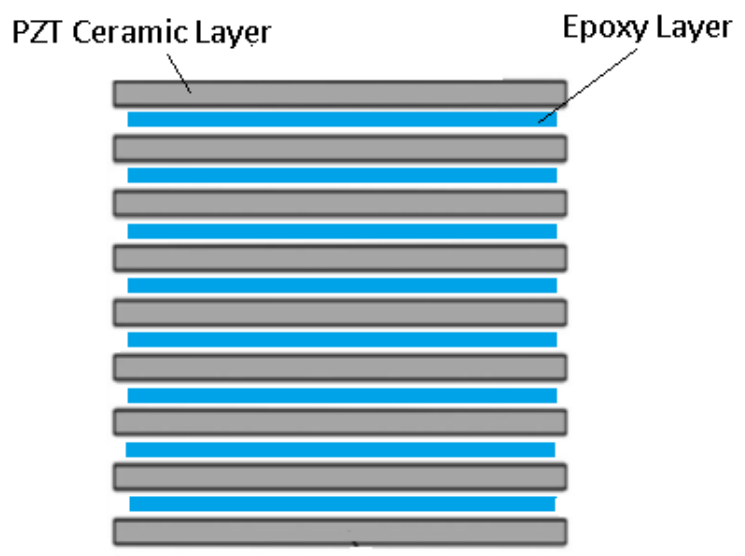

Figure 1.2: Compliant layer adaptive composite stack (CLACS)

In recent years, PZT composites made by mixing piezoelectric particles in a polymer matrix and using dielectrophoresis (DEP) to structure the particles has become more popular ${ }^{21}$. Van den Ende et al. has shown improved piezoelectric properties by using DEP to form chains, or pseudo fibers, in the epoxy matrix to form quasi 1-3 composites compared to randomly dispersed particles with 0-3 connectivity ${ }^{22-24}$. This method keeps manufacturing simple, while allowing the ability to create custom shapes and adjust the mechanical properties of piezoelectric materials ${ }^{24}$. Piezoelectric and dielectric characterization of such materials has been investigated; however, performance of particle composites in mechanical loading has yet to be investigated. A study investigating impact forces of surgeons reported the lowest impact force of 3,597 N while hammering orthopedic implants, a process observed in intramedullary nail insertion ${ }^{25}$. Therefore, less brittle composite piezoelectric materials should be considered for this application.

This study investigates the manufacturing methods of PZT particle composite CLACS for low frequency applications. In addition, it investigates the effect of compliant layers, DEP structuring of particle composite layers, and the combined effect on power generation. A theoretical model for predicting the generating capabilities of PZT particle composite CLACS in low frequency mechanical loading is also investigated. 


\subsection{Electrical Stimulation}

This section discusses the background and development of electrical stimulation for clinical applications. There have been many discoveries of a biological response to electrical stimulation of both hard tissue and soft tissue. The following sections describe the history, function of healing, and findings from current studies.

\subsubsection{History of Electrical Stimulation}

The first discovery the piezoelectric potential of bone was made by Fukada and Yasuda in 1957 , and this discovery has sparked further study of this relationship since then ${ }^{11}$. In the 1970s, the first clinical studies were conducted to evaluate the efficacy of utilizing electrical stimulation to improve the success of fusion in spinal fusion surgeries ${ }^{26}$. Since the 1970s, electrical stimulation has been used as a treatment of non-unions ${ }^{27}$. The optimal current for enhanced bone formation is $20 \mu \mathrm{A}$, and it can be delivered to the site by placing thin wires near the fracture site (cathode) and placing the anode in/on soft tissue nearby ${ }^{26,27}$. The effective stimulation distance from the cathode is described by Kathanovitz to be $5-8 \mathrm{~mm}^{26}$. This effective distance is important for the design of the electrode on any electrical stimulation device in order to maximize healing across the whole fracture or gap.

There are two approved and clinically used products by Zimmer Biomet that currently provide this adjunct therapy of DC electrical stimulation to patients. The first is the $\mathrm{SpF} \circledast$ Implantable Spinal Fusion Stimulator, and it delivers $20 \mu \mathrm{A}$ to bone with a field of influence of $5-8 \mathrm{~mm}^{28}$. This device is utilized in lumbar fusion and has shown to increase success rates by 50\% compared to traditional autograft. To provide the constant DC stimulation, the stimulator has a battery pack placed in the soft tissue about 8 to $10 \mathrm{~cm}$ away from the electrodes ${ }^{28}$. The 
second device by Zimmer Biomet is the OsteoGen ${ }^{\mathrm{TM}}$ Surgically Implanted Bone Growth Stimulator. This device is used to improve the success of long bone fusion and treat or prevent nonunions. This device provides the same constant, DC stimulation and sphere of influence as the SpF. Similarly, the OsteoGen ${ }^{\mathrm{TM}}$ also requires an implanted external battery placed in the soft tissue that will ultimately demand a surgery for removal ${ }^{12}$.

\subsubsection{Bone Tissue}

Fukada and Yasuda discovered that bone becomes electrically polarized when deformed due to two mechanisms: (1) the ionic fluids in bone move about, creating streaming potentials and (2) the piezoelectric properties of collagen molecules in bone tissue ${ }^{11,27}$. According to Martin et al. it is known that the concave surface of a bent bone becomes negatively charged, and the convex surface of bone becomes positively charged. In addition, a bone is negatively charged at the site of a fracture, and bone formation is enhanced when a cathode is nearby ${ }^{27}$. There are a many studies performed to verify this enhanced formation of bone and determine what currents are needed to induce this response.

Some of the first studies of DC stimulation for enhanced bone growth were by Bassett et al. in 1964. They showed that DC stimulation through electrodes in the medullary canal of the femur accelerated bone growth at the electrode location over the course of 21 days $^{29}$. One study by Toth et al. hypothesized that DC stimulation would increase fusion rate of lumbar interbody spinal fusion cages. The DC stimulation was found to significantly improve the stiffness of motion segments in bending, axial rotation, and flexion, and there was a $100 \%$ fusion rate with $100 \mu \mathrm{A}$ stimulation vs. a $27 \%$ fusion rate in the control ${ }^{13}$. Another study by Dejardin et al. hypothesized that increasing the current density delivered would result in faster bone fusion in dogs. It was found that currents of $30 \mu \mathrm{A}$ or greater induced tissue necrosis when delivered 
through stainless steel electrodes because it is delivered as a point charge, instead of a uniformly distributed charge through the entire electrode ${ }^{30}$. However, large currents delivered through titanium electrodes are uniformly distributed and did not cause necrosis. In the past, only overall success of fusion with DC stimulation had been studied ${ }^{13,30,31}$. Dejarden et al. proved bone responds to the amount of current delivered and produces increased fusion rates at higher current densities ${ }^{30}$. The effect of DC stimulation in bone was studied on a cellular level by Bodhak et al. in 2012. Current density was varied from $5 \mu \mathrm{A}$ to $25 \mu \mathrm{A}$ and the highest osteoblast cell density was measured on the Ti surfaces electrically stimulated at $25 \mu \mathrm{A}$. There were $30 \%$ more cells produced compared to the control, non-stimulated Ti surface ${ }^{32}$. In addition, the DC stimulated Ti surfaces significantly increased the osteoblast attachment and growth compared to the control ${ }^{32}$.

\subsubsection{Soft Tissue}

Since the presence of a negatively charged electrode in DC stimulation has been proven to enhance bone healing, many have studied if there are similar benefits to electrically stimulating soft tissue. There are various factors that can delay wound healing or lead to a chronic wounds such as diabetes, vascular insufficiency, age, and nutritional deficiencies ${ }^{33}$. Studies with soft tissue electrical stimulation also arose in the 1970s, and many suggest electrical stimulation reduces infection, improves cellular immunity, increases perfusion, and accelerates wound healing ${ }^{34}$. However, most surgeons do not use this therapy because of the poor understanding of the technology.

There are a few ways to apply this stimulation: direct current (DC), alternating current (AC), high-voltage pulsed current (HVPC), and low-intensity direct current (LIDC). A review of ES for wound healing conducted by Ud-Din et al. evaluated the evidence of success in studies that used these methods ${ }^{35}$. The review included many different studies varying type of current, 
duration, wound type, and dosing of the ES, so there weren't any conclusions proving one method over another. However, a majority of the studies showed significant improvement in wound healing, decreased bacterial infection, and acceleration of healing when compared to a control $^{35}$.

The exact pathway that leads to improved cellular response and accelerated healing has also been hypothesized. In the $1960 \mathrm{~s}$, Becker $^{36}$ determined the existence of direct current surface potentials in living tissues and believed injury caused a shift in current flow in the skin battery, triggering repair. Becker also mentions the effect could be due to $\mathrm{pH}$ or temperature changes when applying ES, but previous studies achieved enhanced healing without changes in either. Another more likely mechanism is electrotaxis, or more specifically, galvanotaxis.

Galvanotaxis is the taxis, or movement of cells and organisms, toward an electrode where DC is the orienting stimulus. Among the cells in the human body, some cells have been found to migrate toward the negative electrode (e.g., fibroblasts, keratinocytes, neural crest cells, and many epithelial cell types $)^{37}$, and other cells migrate toward the positive electrode (e.g., corneal endothelial cells and human vascular endothelial cells) ${ }^{37}$.

A recent study by Joseph Hoare et al. studied human macrophage characteristics and movement in the presence of DC stimulation. These cells are important to defense and tissue repair following injury, infection, or malignancy ${ }^{38}$. Macrophages facilitate healing by resolution of inflammation and phagocytosis of apoptotic cells, cell debris, and microbes. In addition, they secrete factors such as cytokines that promote angiogenesis, extracellular matrix, and more in order to facilitate regeneration of the tissue ${ }^{39}$. Hoare et al. stimulated macrophages with 5-450 $\mathrm{mV} / \mathrm{mm}$ for $2 \mathrm{~h}$. In the presence of these EFs, they found $85 \%$ of the cells migrated toward the positive electrode, macrophages were reorganized resulting in a new shape, actin distribution 
was significantly increased, phagocytosis significantly increased, and enhances cytokine secretion $^{39}$.

Another study by Francis Lin et al. found that lymphocytes, one of the body's main types of immunity cells, migrate toward a negative electrode through galvanotaxis ${ }^{39}$. It is suggested that good placement of a negative electrode can improve infection prevention and help close open wounds through the migration of epithelial cells ${ }^{40}$. Since many studies have found benefits to both positive and negative DC stimulation in wound healing, it may be beneficial to stimulate both asynchronously or in an alternating fashion. One of the very first studies of electropotentials at an incision healing site by Burr et al. found that the potentials are initially positive, but become negative on the fourth day of healing and remained negative ${ }^{41}$. Even though the difference types of ES are all shown to improve and enhance healing, the characteristics of the cells and healing process may point to the use of alternating current for overall better healing. By alternating the polarity of the electrodes, macrophages become more efficient and migrate to the wound while positive, and the fibroblasts, epithelial cells, and white blood cells migrate while the electrode is negative.

\subsection{MEMS}

With the continual improvement of semiconductor technologies, small electronic devices are becoming more prevalent and useful as the technology advances. These devices, or microelectromechanical systems (MEMS), require very low amounts of power and can be utilized for sensing, transmitting, or portable electronics ${ }^{7,42}$. However, there are some applications where these MEMS are placed where remote power is required, such as inside the human body ${ }^{7,42}$. Even though batteries have also advanced in efficiency, they have to be removed or replaced at the end of their service life ${ }^{42}$. 
According to Li et al., the level of power consumption for MEMS lies in the $\mathrm{mW}$ or $\mu \mathrm{W}$ range and the circuit needs to be small ${ }^{42}$. In order to avoid the use of batteries and provide the power necessary for these devices, piezoelectric generators can be utilized. These materials convert usable mechanical energy into electrical energy (this will be discussed more in the following section). This is specifically useful for sensors or electrical stimulation devices in the human body. One study utilized PZT wafers inside shoes to convert mechanical energy from walking into electrical energy ${ }^{43}$. This system achieved $1.3 \mathrm{~mW}$ at a walking frequency of $0.8 \mathrm{~Hz}$. Another study by Goetzinger et al. utilized PZT fiber-epoxy composites to replace implantable batteries for an electrical stimulation device ${ }^{18}$. At physiological loading and a walking rate of 2 $\mathrm{Hz}$, the PZT generator achieved $1.13 \mathrm{~mW}$. Therefore, these piezoelectric generators can successfully be implemented to power MEMS in vivo, without the need for removal surgery or replacement.

\subsection{Piezoelectric Materials}

\subsubsection{Piezoelectric Ceramics}

When certain crystalline ceramics are subjected to a mechanical force, they become electrically polarized. The tension and compression on the ceramic generate voltages of opposite polarity in proportion to the applied force ${ }^{44}$. According to APC International Ltd., this characteristic was discovered in 1880 by Jacques and Pierre Curie. They also found that the application of an electric field caused the material to lengthen or shorten according to the polarity of the field ${ }^{44}$. This piezoelectric property of these ceramics has allowed us to utilize these materials as sensors, actuators and generators. According to Platt et al., one of the earliest uses of piezoelectric materials was the development of the first SONAR system in 1917 by Langevin $^{7}$. Currently, piezoelectric sensors that measure force, pressure, and acceleration are 
commercially available. In addition to utilizing piezoelectrics for sensing, the same materials can convert mechanical energy into electrical energy ${ }^{7}$.

These energy harvesting and sensing materials can be used to power MEMS, devices that run on extremely low power where remote power is required. For medical devices that need power or provide electrical stimulation, implanted batteries are used and require an invasive surgery, have limited lifespan, and eventually need replaced or removed ${ }^{45}$. These batteries create a higher chance for infection and discomfort to the patient. Therefore the use of piezoelectric materials for energy harvesting can be very useful to allow self-powered MEMS or electrical stimulation devices. Some studies have already begun to apply piezoelectric materials to medical applications to generate the electricity to promote tissue growth ${ }^{15}$. The following section contains the theory behind these materials and how different configurations, piezoelectric materials, and manufacturing methods provide unique benefits for different applications.

\subsubsection{Piezoelectric Theory}

Piezoelectric ceramics are crystalline materials that generate electrical potentials (voltages) when a mechanical stress is applied. The stress distorts an internal dipole moment that produces the voltage in direct proportion to the applied forces (Figure 1.3) ${ }^{7}$. The direct piezoelectric effect

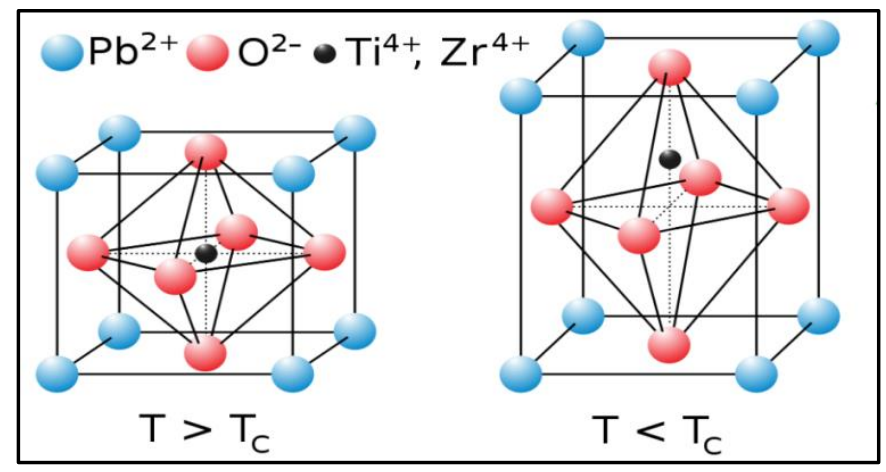

Figure 1.3: The perovskite structure of Lead Zirconate Titanate (PZT), showing the structure may be polarized when below the Curie temperature (TC). (Public domain, commons.wikipedia.org, Labeled for Reuse). 
is observed when a piezoelectric material produces a voltage when subjected to a mechanical stress that is proportional to said stress. The inverse piezoelectric effect is observed when a piezoelectric material strains proportional to an applied electric field ${ }^{44,46}$. In order to get the internal diploe moment in the perovskite structure, the material must be poled. Ceramics like PZT must be made piezoelectric by applying a poling treatment involving exposing the material to a strong electric field at a temperature just below the Curie point. This electric field is typically around $1-3[\mathrm{kV} / \mathrm{mm}]$ and will result in a net polarization of the domains and anisotropy of the material (Figure 1.4) $)^{44,46}$.

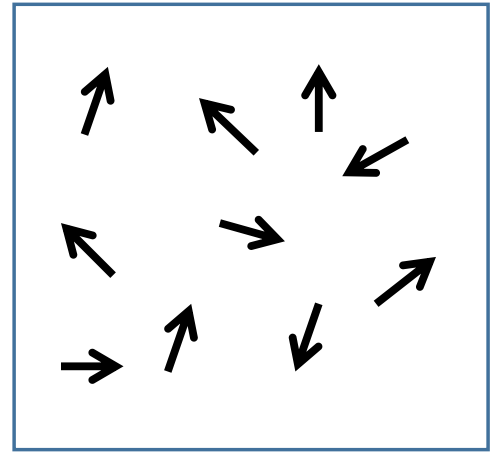

Before Poling

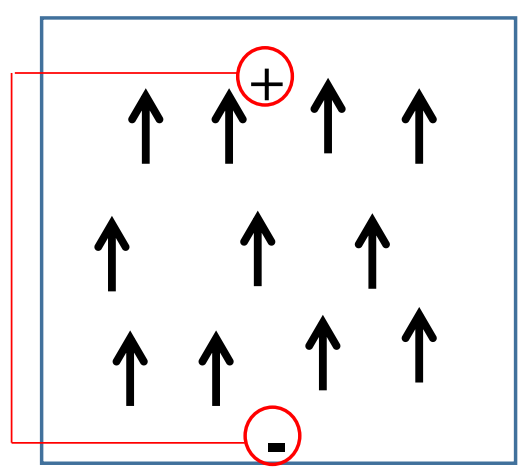

During Poling

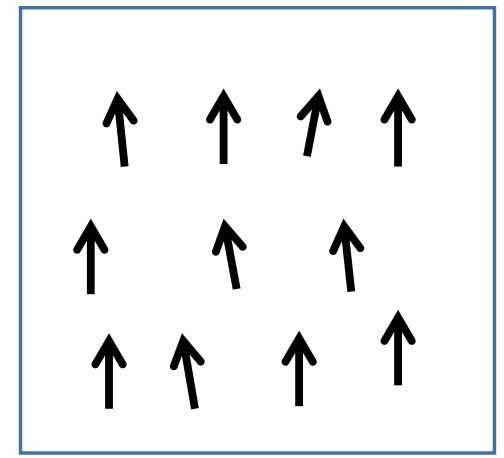

After Poling

Figure 1.4: The poling treatment for PZT showing the random alignment of domains (left) and the resulting net polarity and anisotropy (right).

Lead zirconium titanate is one of the most commonly used piezoelectric ceramics due to its superior piezoelectric properties and its relatively high Curie temperature ${ }^{44,46}$. The ceramic is formed by taking fine powders of metal oxides, calcining at high heat to form a uniform powder, mixing it with an organic binder, pressing it into molds, and sintering to achieve the dense crystal structure $^{44}$. The resulting poled ceramic is anisotropic (both mechanically and electrically); 
therefore, the properties are given two subscripts to define the property with respect to poling direction and loading direction.

Electrodes are typically applied to the top and bottom surfaces of the poled piezoelectric generators to harvest electric potentials. However, these generators have a frequency dependent, effective resistance, or impedance, that affects the efficiency of power generation. When exposed to a cyclic electric field or mechanical load, there is a frequency at which piezoelectric ceramics most efficiently operate called the resonance frequency ${ }^{44,46}$. This is the frequency at which piezoelectric properties are measured, and it typically lies in the $\mathrm{kHz}$ or $\mathrm{MHz}$ range. The use of these materials in low frequency $(0-100 \mathrm{~Hz})$ applications is desired; however, the impedance of PZT at off-resonance, low frequencies is too high for use in small electrical devices ${ }^{7,42}$. Platt et al. showed that a stack of PZT elements, mechanically in series and electrically in parallel, significantly lowers source impedance ${ }^{7}$. This method of manufacturing is able to produce larger output currents and better match the impedance of the electrical device compared to the same volume of PZT in a monolithic stack ${ }^{7,42}$.

\subsection{Piezoelectric Composites}

\subsubsection{PZT Composites}

Traditional PZT ceramics as described previously are very brittle and cannot be implemented in high strain environments ${ }^{47,48}$. In addition, these purely ceramic materials are difficult to shape and process ${ }^{22,48}$. Piezoelectric composites have been developed by mixing a piezoelectric ceramic phase into a polymer matrix. These composite materials can be tuned to provide desired mechanical, thermal, dielectric and electroactive properties ${ }^{22,23}$. In such composites, the connectivity of the two phases is critical to the electrical and mechanical performance of the material $^{46}$. This connectivity is described as the interspatial relationships in multiphase 
materials ${ }^{16,22}$. Newnham describes 10 different patterns of connectivity between two phases that can be described by two numbers ${ }^{16}$. The first number describes the connectivity of the filler and the second describes the connectivity of the matrix. For example, a composite composed of randomly distributed ceramic particles in a polymer matrix is indicated as $0-3$. This is because the particles do not have interconnectivity, while the polymer phase is connected in all 3 dimensions. A composite composed of long ceramic fibers within a polymer matrix is indicated as $1-3$, since the ceramic is now connected in 1 dimension ${ }^{16,22}$.

\subsubsection{Dielectrophoresis}

The 1-3 composites, or PZT fibers aligned within matrix epoxy outperform 0-3 composites due to higher coupling coefficients and voltage sensitivity, but are very difficult to manufacture and are not suitable for large scale manufacturing ${ }^{19,46,48}$. The 0-3 composites lack in piezoelectric properties due to limited connectivity and differences in permittivity between the polymer and ceramic inclusions ${ }^{48}$. However, a new method of processing particles within matrix materials has been significantly studied and developed within the last 10 years. DEP is an effect where a particle is carried, or receives a force, as a result of its dielectric properties ${ }^{21}$. This method of arranging or moving suspensoid particles has been implemented in biosensors, cell therapeutics, microfluidics, and particle filtration. Van den Ende et al. successfully utilized DEP to perform the in situ structuring of PZT particles to create quasi 1-3 composites. Initially, the particles are oriented randomly, and an alternating electric field is applied as the matrix epoxy cures. The DEP force moves the dielectric particles to form chains, oriented in the direction of the electric field (Figure 1.5) 22,48 .

Studies have reported that DEP structuring increases the permittivity and piezoelectric properties such as $\mathrm{d}_{33}$ and $\mathrm{g}_{33}$ of PZT-epoxy composites ${ }^{22,23}$. It is understood that an increase in 
the volume fraction of PZT to epoxy with result in a better piezoelectric properties. However, Van den Ende et al. discovered that the effect of DEP is enhanced at lower volume fractions (10$20 \%$ ). There was still a $25 \%$ enhancement of the $\mathrm{d}_{33}$ at $50 \%$ volume fraction. It was determined that the particles are more freely able to move into the chains at lower contents, but the high volume fractions still achieve a higher degree of parallel connectivity from $\mathrm{DEP}^{22}$.
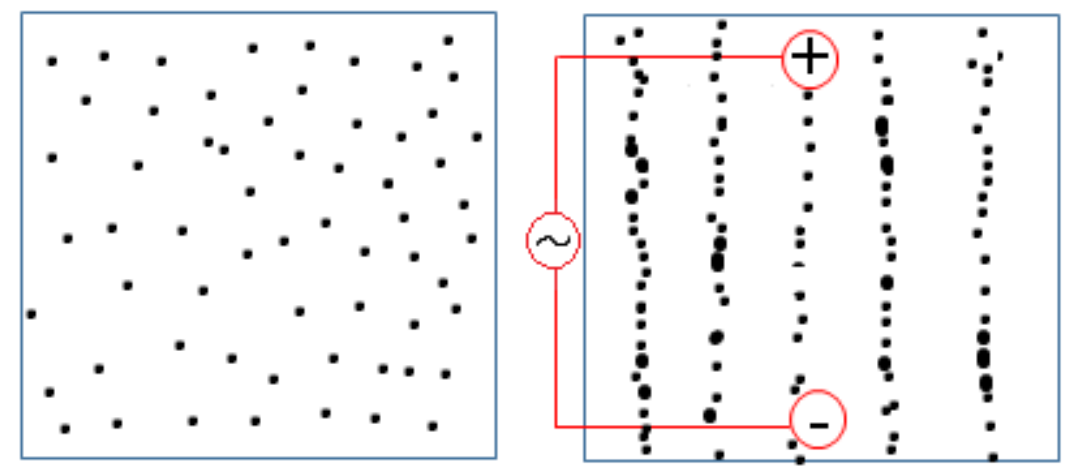

Figure 1.5: Dielectrophoretic structuring of particles showing the randomly dispersed particles (left) and the aligning of particles under an alternating electric field (right).

\subsubsection{Composite Poling}

There are two main methods utilized for the poling of piezoelectric materials. The traditional method is called parallel plate-contact polarization, where the piezoelectric material is in contact with the poling electrodes (Figure 1.6) ${ }^{49}$. The material is often submerged in an oil bath to isolate it from air and is poled with a direct electric field of $2-3 \mathrm{kV}^{44,48}$. However, this method is not very successful with multiphase piezoelectric composites. The dielectric constant of the epoxy is significantly lower than the ceramic particles, almost shielding the particles from high electric fields. Therefore, very large electric fields are required to pole the PZT-epoxy composites ${ }^{50}$. These large electric fields end up causing dielectric breakdown of the material, creating a short circuit across the electrodes ${ }^{50,51}$. 
To overcome these issues and successfully pole multiphase piezoelectric composites, the corona discharge poling method is used. In this method, a large DC potential is applied to a set of needles, or corona points, which intensify the current density, causing the surrounding air to be ionized $^{51,52}$. Only one side of the sample is electrode and placed on the ground plate, and the

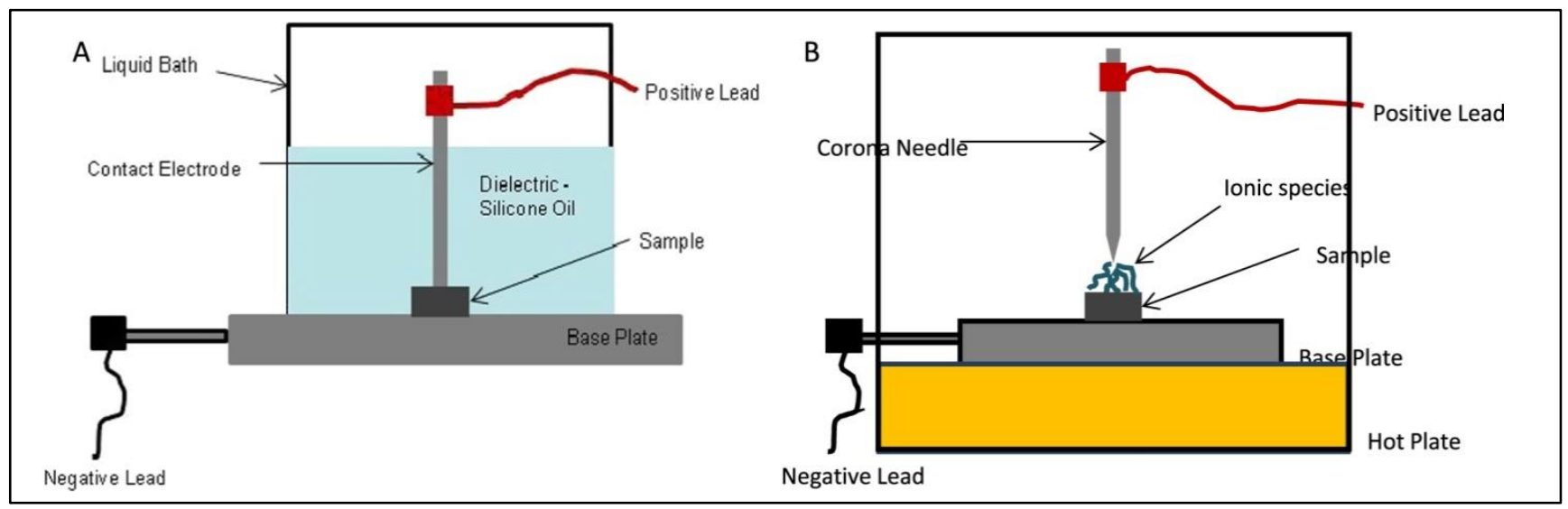

Figure 1.6: Diagrams for setup of both the parallel plate-contact method (A) and the corona discharge poling method (B). @ IOP Publishing. Reproduced with permission. All rights reserved.

electric charge form the needle prays onto the unelectroded surface ${ }^{52}$. As the ionic species are attracted to the surface, charge builds until a sufficient surface charge density is met and ions flow toward the base plate, aligning the dipoles of the PZT phase ${ }^{51,52}$. Figure 1.6 shows the poling setup for both methods. The corona discharge method allows poling at the necessary high voltages $\left(4-10 \mathrm{kVmm}^{-1}\right)$ without dielectric breakdown. 


\section{Chapter 2: Smart Materials and Structures Paper}

This section contains a manuscript for eventual submission to Smart Materials and Structures, Submission planned for September 2018.

This manuscript developed a theoretical model to predict the power generation capabilities and investigate the effect of compliant layer thickness and dielectrophoretic structuring on power in a CLACS generator. ZR Pessia had primary responsibility for study design, composite manufacturing, theoretical model development, data analysis, writing of the manuscript, and editing with recommendations from fellow co-authors. 


\title{
Power amplification via compliant layer interdigitation and dielectrophoretic structuring of PZT particle composites
}

\author{
Zach Pessia ${ }^{1}$, Craig Cunningham², Ember Krech ${ }^{2}$, Elizabeth A Friis ${ }^{1,2}$ \\ ${ }^{1}$ Department of Mechanical Engineering, University of Kansas, Lawrence, KS \\ ${ }^{2}$ Bioengineering Graduate Program, University of Kansas, Lawrence, KS
}

E-mail: 1friis@ku.edu

\begin{abstract}
The evolution of portable electronics has led to recent popularity of piezoelectric materials for energy harvesting, especially for devices deployed remotely or in vivo. The efficiency of piezoelectric generators harvesting energy from the human body is lacking due to off-resonance loading. Piezoelectric ceramics are expensive to manufacture and are dense, brittle, and difficult to use in high strain environments. Piezoelectric composites composed of ferroelectric particles distributed in a polymer matrix are desirable due to low cost and tunable properties. In this study, Compliant Layer Adaptive Composite Stacks (CLACS) made with thin piezoelectric composite layers structured by dielectrophoresis (DEP) were investigated to increase the energy harvesting efficiency at low frequencies. To predict power generation capabilities, a theoretical model was developed by using established particle composite models in conjunction with a shear lag structural mechanics model for CLACS. Granular composite discs of lead zirconate titanate particles in an epoxy matrix were manufactured at a 50\% volume fraction and structured by DEP, if applicable. CLACS were manufactured using ten composite discs and two compliant layer thicknesses. The stacks were electromechanically tested by varying load, frequency, and resistance. Experimental results showed a significant increase in power amplification with DEP structured discs and compliant layers. In addition, the theoretical model accurately predicts power production for both 0-3 and 1-3 CLACS at low frequencies. DEP structured particle composite CLACS can provide a method of energy harvesting for devices in remote locations, especially in low frequency high strain environments.
\end{abstract}

Keywords: Piezoelectric, composite, particles, electrical stimulation, MEMS, dielectrophoresis 


\subsection{Introduction}

In recent years, the use of piezoelectric materials for energy harvesting has grown with the evolution of portable electronics that can be deployed in remote locations for extended lifespans ${ }^{1}$. The need for piezoelectric power generators is driven by the limited lifespans of batteries and the need to replace them periodically ${ }^{1,2}$. Piezoelectric generators harness ambient energy and convert it to electrical charge that can be emitted or stored ${ }^{1}$. These generators have potential to be utilized in remote applications such as microelectromechanical systems (MEMS) devices or in vivo sensors.

Piezoelectric materials produce maximum power at the resonance frequency, typically observed in the kilohertz or megahertz range ${ }^{2}$. Maximum power is characteristic of high voltages due to high source impedance, making it difficult to integrate monolithic generators into a small circuit, especially at low frequencies ${ }^{3}$. Multilayer cofired generators, composed of thin piezoelectric ceramics stacked mechanically in series and connected electrically in parallel, can be utilized to produce the same power as a monolithic piezoelectric generator, while substantially

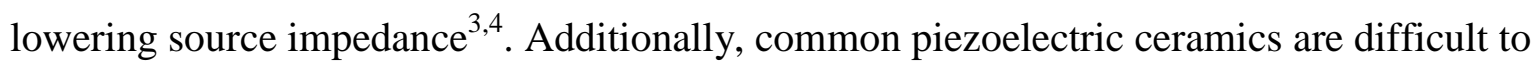
process, dense, and brittle, making their use in high strain environments challenging ${ }^{5-7}$. This results in costly, fragile, materials that are less suitable for large scale manufacturing, leading to few piezoelectric manufacturers with high price points for custom piezoelectric ceramics designs 5

Piezoelectric composites offer an alternative, cost effective solution to these issues. These composites are made using a particulate piezoelectric ceramic phase dispersed within a polymer matrix $^{5,6,8}$. They provide tunable mechanical properties and allow for custom molded shapes, beneficial for unique applications such as MEMS or in vivo sensing. However, these composites 
display low piezoelectric properties due to limited connectivity of the ceramic phase, caused by large differences between dielectric properties of the polymer and ceramic inclusions $5,8,9$. Newnham describes 10 different patterns of connectivity between two phases that can be described by two subscript numbers ${ }^{8}$. A composite composed of randomly distributed piezoelectric particles within a polymer matrix is indicated as $0-3$, since the particles have no connectivity, while the polymer is connected in all three dimensions. A composite composed of piezoelectric fibers aligned within a polymer matrix is indicated as 1-3, since the ceramic fibers have connectivity in one dimension. 1-3 composites outperform 0-3 composites due to higher coupling coefficients and voltage sensitivity, but can be difficult and expensive to manufacture ${ }^{5,10,11}$.

Dielectrophoresis (DEP) is a phenomenon where a particle is carried, or receives a force, as a result of its dielectric properties ${ }^{12}$. In PZT-epoxy composites, the application of an electric field to the uncured thermoset epoxy aligns the dispersed particles into chains by means of dielectrophoresis $^{5,7}$. In 2010, Van den Ende et al. utilized DEP to perform is situ structuring of PZT particles into aligned chains to create quasi 1-3 piezoelectric composites, displaying improved piezoelectric properties including the permittivity, piezoelectric charge constant $\left(\mathrm{d}_{33}\right)$, and piezoelectric voltage constant $\left(\mathrm{g}_{33}\right)^{5,6,11}$. This work showed that higher connectivity can be achieved at higher particle volume fractions at the expense of reducing the strain of failure ${ }^{5,13}$. Therefore, additional measures may be taken to increase toughness in applications where maximum power is desired.

A recent study by Krech et al. investigated a method of interdigitating polymer layers between piezoelectric ceramic discs in order to develop a tough piezoelectric stacked generator. The addition of the softer layer in Compliant Layer Adaptive Composite Stacks (CLACS) was 
also found to result in an increased ability of the stacked generator to produce power. For one specimen type, the addition of a compliant layer between thin PZT layers resulted in a $61 \%$ increase in power production compared to a conventional stack with the same volume of $\mathrm{PZT}^{10}$. This power amplification was attributed to an increase in sensitivity to through-thickness compression caused by positive in-plane strains and was successfully modeled with a shear lag structural mechanics model ${ }^{14}$.

In the present study, the ability to generate power with CLACS made with thin 0-3 and 1-3 particle composite layers is investigated. Power generation is characterized under mechanical loads and low frequencies characteristic of human body motion. The Yamada model and Van den Ende models are used to compare experimental results to theoretical models for 0-3 and 1-3 particle composites respectively. The Krech et al. theoretical model for power amplification is used in this work to predict and compare the effect of compliant layers. It was hypothesized that the addition of a polymer compliant layer between PZT composite discs would amplify power production as compared to the composite stack without compliant layers. In addition, it was hypothesized that structuring composite discs by means of DEP would increase power production, given constant PZT volume, size, surface area, and thickness. It was expected that the relationships between load, frequency, and PZT power production would be consistent with previous studies characterizing piezoelectric materials under physiological mechanical loading conditions.

\subsection{Materials and Methods}

\subsubsection{Composite Manufacturing}

Navy Type II, PZT-5 particles were chosen for this study (American Piezo Ceramics, Mill Hall, PA). This material was chosen due to its high piezoelectric charge constant (400 pC/N) and 
high coupling factor $(0.72)$. The powder was sintered at $700{ }^{\circ} \mathrm{C}$ for 1 hour to burn off the organic binder holding the particles in sphere agglomerates. The sintered powder was then ground with a mortar and pestle to disaggregate the agglomerate spheres into individual $0.5 \mu \mathrm{m}$ particles. A two component, medical grade epoxy (EPO-TEK® 301, Epoxy Technology, Billerica, MA) was used to form the composite. The epoxy is viscous at room temperature and has a low dielectric constant $(4.00$ at $1 \mathrm{kHz})$ which is desirable for $\mathrm{DEP}^{7}$. After combining the two part epoxy, particles were added to achieve a $50 \%$ volume fraction (VF) of ceramic content, and mixed for 15 minutes with a spatula at about $60 \mathrm{rpm}$. The composite slurry was then placed into a $0.4 \mathrm{~mm}$ thick Teflon mold, consisting of 30, $10 \mathrm{~mm}$ diameter circle cut-outs (Figure 2.1).

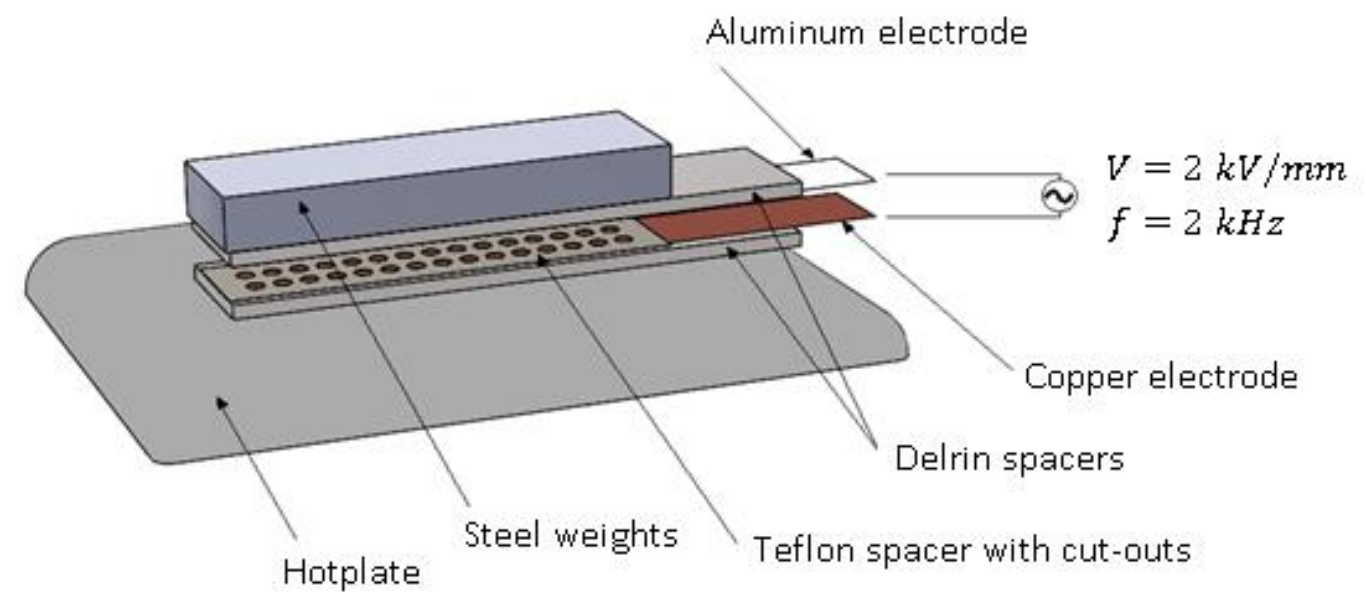

Figure 2.1: Schematic of mold setup for dielectrophoresis processing Immediately after the setup was placed on the hotplate, a function generator (B\&K Precision, Yorba Linda, CA) coupled to a high voltage amplifier (10/10B-HS, TREK, Lockport, NY) was used to structure the particles in the uncured composite by means of dielectrophoresis. An electric field of $2 \mathrm{kV} / \mathrm{mm}$ and frequency of $2 \mathrm{kHz}$ was applied across the electrodes for 3 hours at $65{ }^{\circ} \mathrm{C}$ then applied for 1 hour while cooling to room temperature. The non-structured, or randomly dispersed, samples were manufactured in the same fashion without applying an electric 
field by curing at room temperature for 24 hours. The fully cured discs were sanded with 250 grit sand paper to $0.4 \pm 0.03 \mathrm{~mm}$ thickness to remove the top epoxy layer. After cleaning and drying the discs, a copper electrode of $9.2 \mathrm{~mm}$ diameter and $25 \pm 5 \mu \mathrm{m}$ thickness was sprayed onto one side (843AR Super Shield ${ }^{\mathrm{TM}}$, MG Chemicals, Surrey, BC). The discs were poled using the corona poling method by applying $15 \mathrm{kV} / \mathrm{mm}$ to a needle $7.5 \mathrm{~mm}$ above the surface of the unelectroded disc for 30 minutes at $100{ }^{\circ} \mathrm{C}$. The discs were then cooled to room temperature in the presence of the poling field for 30 minutes then stored with ends shorted to remove any trapped charges form polarization or impurities. Lastly, the second electrode was sprayed onto each disc and poling success was verified by pyroelectric analysis of each disc.

\subsubsection{Specimen Manufacturing}

CLACS were manufactured using two different compliant layers thicknesses $(0.0 \mathrm{~mm}$ and $0.8 \mathrm{~mm}$ ) for both DEP structured and random particle composite discs ( $\mathrm{n}=5$ in each group, $\mathrm{N}=20$ ). The thicknesses were selected to evaluate ratio of compliant layer thickness to PZT composite layer thickness and its effect on power generation ${ }^{14}$. The CLACS were manufactured by stacking 10 composite discs with cured epoxy layers adhered between each disc, if applicable. The stacks were encapsulated in epoxy, keeping volume of PZT, thickness of top and bottom encapsulation, and surface area constant. Before encapsulation, the 10 discs were electrically connected in parallel with $2 \mathrm{~mm}$ wide copper foil strips and conductive epoxy (EPO-TEK®) H20E, Epoxy Technology, Billerica, MA). Once cured, the chains of 10 discs were folded and stacked using an accordion method ${ }^{10}$, utilizing a minimal amount of epoxy to adhere each layer placing the discs mechanically in series. This minimal amount epoxy layer for adhesion $(\sim 0.1$ $\mathrm{mm}$ ) will be considered in theoretical modeling; however, the stacks without additional, interdigitated compliant layers will be labeled as $0.0 \mathrm{~mm}$. For the $0.8 \mathrm{~mm}$ compliant layer 
groups, $11 \mathrm{x} 11 \mathrm{~mm}$ slices of cured epoxy of $0.8 \mathrm{~mm}$ thickness were adhered between each disc to create CLACS. Lastly, each stack was encapsulated with EPO-TEK 301 and electrical connectivity of all 10 discs was verified with impedance measurements.

\subsubsection{Electromechanical Testing}

The CLACS were tested electromechanically to compare voltage produced at varying compressive mechanical loads, frequencies, and circuit resistances. The loading conditions were selected to best characterize the behavior of the composite CLACS for use in the human body. An MTS MiniBionix 858 (MTS, Eden Prairie, MN) was used to apply a $1200 \mathrm{~N}$ compressive preload before applying cyclic compression at two load amplitudes of $500 \mathrm{~N}$ and $1000 \mathrm{~N}$ at varying frequencies of $1 \mathrm{~Hz}, 2 \mathrm{~Hz}, 3 \mathrm{~Hz}$, and $5 \mathrm{~Hz}$. In order to characterize the performance of the composite stacks for circuit design, each loading condition was tested across a shunting resistance sweep from $2 \mathrm{M} \Omega$ to $300 \mathrm{M} \Omega$. A MATLAB (Mathworks, Natick, MA) code was written to obtain the RMS voltage, $\mathrm{V}_{\mathrm{RMS}}=\mathrm{V}_{\text {out }} / \sqrt{2}$, and average maximum power for each loading condition and resistance, $\mathrm{P}=\mathrm{V}_{\mathrm{RMS}}{ }^{2} / \mathrm{R}$. A Wilcoxon Rank Sum test was used to compare power production as a function of compliant layer thickness and particle structuring $(\alpha=0.05)$.

\subsection{Results}

\subsubsection{Piezoelectric Characterization}

The analytical model used to determine the piezoelectric properties of $0-3$ or random composites was proposed by Yamada et al. in $1979^{9}$. The composite is assumed to have random distribution of particles within a polymer matrix wherein the dielectric constant, $K$, and piezoelectric charge constant, $d_{33}$, in the poling direction are determined by:

$$
\mathrm{K}_{\text {random }}=\varepsilon_{\mathrm{M}} *\left(1+\frac{\eta \Phi\left(\varepsilon_{\mathrm{m}}-\varepsilon_{\mathrm{c}}\right)}{\eta \varepsilon_{\mathrm{m}}+\left(\varepsilon_{\mathrm{c}}-\varepsilon_{\mathrm{m}}\right)(1-\Phi)}\right)
$$




$$
\mathrm{d}_{33_{\text {random }}}=\frac{\Phi \alpha \eta \mathrm{K}_{\text {random }} \mathrm{d}_{33_{\mathrm{c}}}}{\eta \mathrm{K}_{\text {random }}+\varepsilon_{\mathrm{c}}-\mathrm{K}_{\text {random }}}
$$

where $\varepsilon$ is the dielectric constant, $\eta$ is the inverse of the depolarization factor, and $\Phi$ is the volume fraction, $\alpha$ is the poling ratio, and the subscripts $m, c$, and random refer to the polymer matrix, ceramic, and 0-3 properties respectively.

The Bowen model is used to determine piezoelectric properties of the structured composites ${ }^{15}$. It does this by assuming DEP has formed chains or fibrils of particles in line with the electric field surrounded by matrix epoxy. The equation for the dielectric constant for structured or 0-1 composites was be determined by:

$$
\mathrm{K}_{\text {structured }}=\Phi_{\mathrm{c}}\left(\frac{\mathrm{R} \varepsilon_{\mathrm{c}} \varepsilon_{\mathrm{m}}}{\varepsilon_{\mathrm{c}}+\mathrm{R} \varepsilon_{\mathrm{m}}}\right)+\left(1-\Phi_{\mathrm{c}}\right) \varepsilon_{\mathrm{m}}
$$

where $R$ is the ratio of average particle size to the effective interparticle distance ${ }^{15}$. A value of 6.2 for $\mathrm{R}$ was obtained in order to achieve best fit of experimental data to the Bowen model. Beyond dielectric characterization, Van den Ende et al. developed an extension to Bowen's model in order to characterize the $d_{33}$ of structured composites. This was achieved by modeling the particle chains as two capacitors in series electrically and two springs in series mechanically ${ }^{6}$ and is derived by:

$$
\mathrm{d}_{33_{\text {structured }}}=\frac{(1+\mathrm{R})^{2} \varepsilon_{\mathrm{m}} \Phi_{\mathrm{c}} \mathrm{d}_{33} \mathrm{Y}_{\mathrm{c}}}{\varepsilon_{\mathrm{c}}+\mathrm{R} \varepsilon_{\mathrm{m}}\left[\left(1+\mathrm{R} \Phi_{\mathrm{c}}\right) \mathrm{Y}_{\mathrm{c}}+\left(1-\Phi_{\mathrm{c}}\right) \mathrm{R} Y_{\mathrm{m}}\right.}
$$

where $Y_{c}$ and $Y_{m}$ are the elastic moduli of the ceramic and epoxy phases respectively in the thickness direction ${ }^{6}$. To model the effect of the interdigitated compliant layers, the previously stated models were combined with the CLACS model by Krech et al.. The baseline power from a piezoelectric stack without compliant layers $\left(P_{0}\right)$ can be determined as a function of work $(W)$, frequency $(f)$, and electromechanical coupling factor $\left(k_{33}\right)$ by: 


$$
\mathrm{P}_{0}=\mathrm{W} * \mathrm{f} * \mathrm{k}_{33}^{2}
$$

The modulus of the particle-epoxy composite is determined by the Halpin-Tsai model for spherical particle composites:

$$
\mathrm{E}_{\text {Composite }}=\mathrm{E}_{\mathrm{M}} *\left(\frac{1+\zeta \mathrm{Nf}}{1-\mathrm{Nf}}\right)
$$

where $\zeta$ and $N$ are factors used to describe the influence of geometry ${ }^{16}$. For the randomly dispersed spherical particles, the appropriate $\zeta$ value was used. However, in order to best characterize the DEP structured composites, parameters for aligned continuous fibers were used, since the structuring produces fiber-like reinforcement in the thickness direction. The model by Krech et al. for the power amplification due to the addition of compliant layers in piezoelectric stacks is given by the equation:

$$
\mathrm{AF}=\frac{\mathrm{P}}{\mathrm{P}_{0}} \sim 1+\left|\frac{\mathrm{d}_{31}}{\mathrm{~d}_{33}}\right| c \sqrt{\left(\frac{\mathrm{t}_{\mathrm{cl}}}{2 \mathrm{t}_{\text {composite }}}\right)\left(\frac{\mathrm{E}_{\text {composite }}}{\mathrm{E}_{\mathrm{CL}}}\right)}
$$

where $t$ is thickness, $E$ is the modulus, c is the contiguity factor, and $d$ is the respective charge constant ${ }^{14}$. The ratio of $d_{31}$ to $d_{33}$ is used in the amplification factor due to the enhanced displacement observed in CLACS. This is equivalent to the ratio of $g_{31}$ to $g_{33}$, a more appropriate property with respect to electrical energy produced from input mechanical stress. The contiguity factor was obtained to be 0.62 in order to refine the amplification factor and account for variability in manufacturing. Equations 2.5 and 2.7 can be combined to predict the electromechanical coupling factor of the composite, resulting in the equation: 


$$
\mathrm{k}_{33}=\sqrt{\mathrm{k}_{33_{0}}{ }^{2}\left(1+\left|\frac{\mathrm{d}_{31}}{\mathrm{~d}_{33}}\right| \mathrm{c} \sqrt{\left(\frac{\mathrm{t}_{\mathrm{cl}}}{2 \mathrm{t}_{\text {composite }}}\right)\left(\frac{\mathrm{E}_{\text {composite }}}{\mathrm{E}_{\mathrm{CL}}}\right)}\right)}
$$

Experimental results for maximum power as a function of compliant layer thickness (1000 N, 5

$\mathrm{Hz}, 40.5 \mathrm{M} \Omega$ ) were plotted against theoretical results. Good correlation between both the 0-3 and 1-3 composites and their associated models was observed (Figure 2.2). Experimental values for 0-3 samples slightly outperform the model but are very reasonable. The theoretically modeled values for maximum power produced are all within $6 \%$ error of the experimental values achieved by each structuring and compliant layer combination. Electromechanical coupling factor was calculated from experimental maximum power and plotted against theoretical $\mathrm{k}_{33}$ (Figure 2.3).

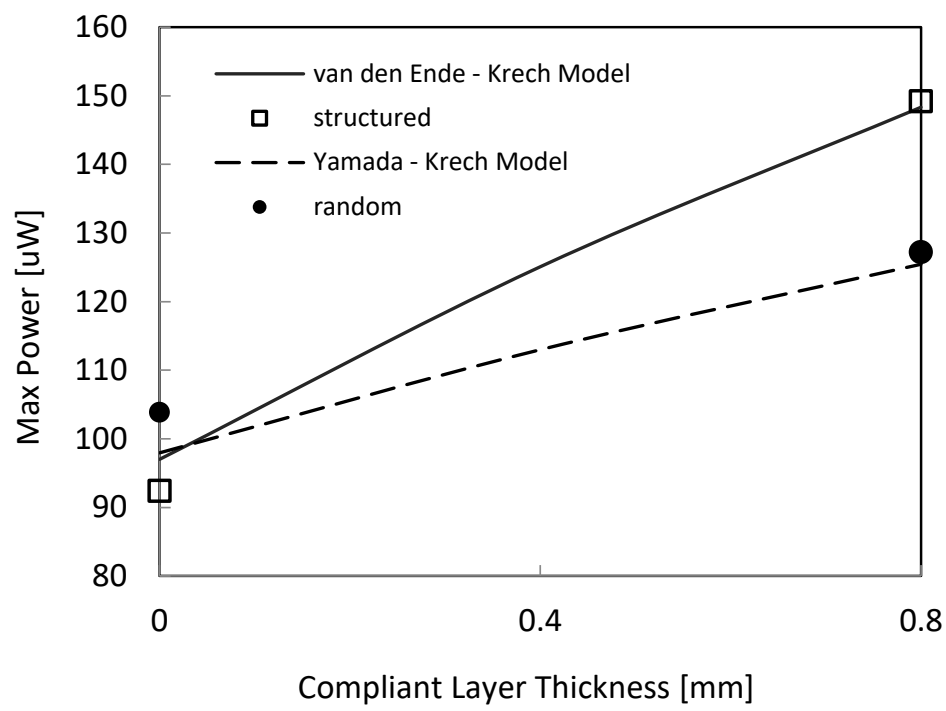

Figure 2.2: Maximum power of theoretical models for structured and random CLACS and the experimental results for average maximum power. 


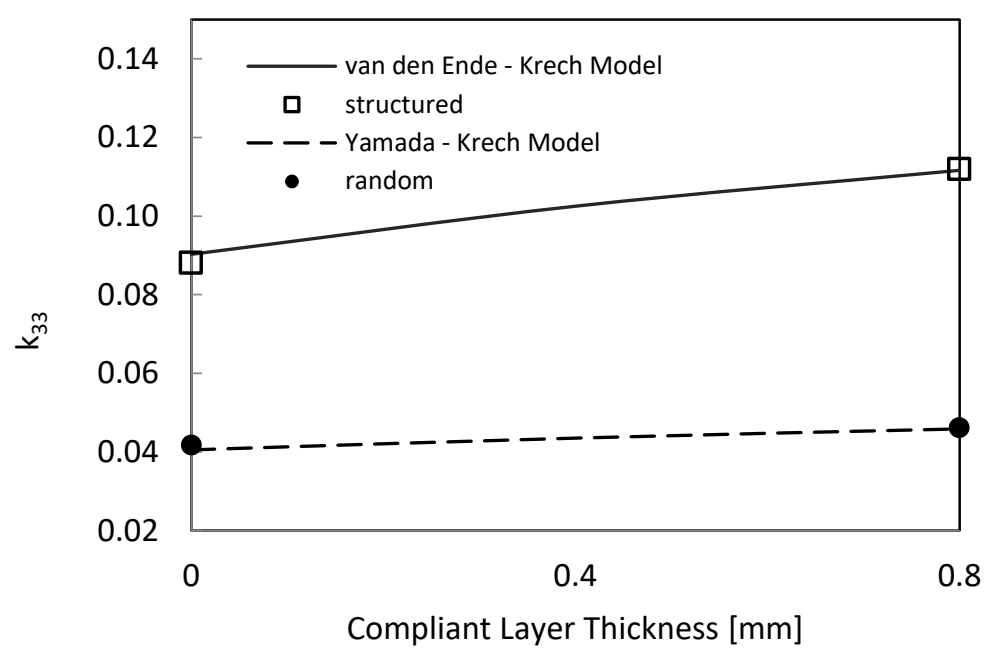

Figure 2.3: Electromechanical coupling factor $\left(\mathrm{k}_{33}\right)$ of theoretical models and experimental results for both random and structured CLACS.

In all of the models, it is assumed that the individual particles have the same properties as the bulk ceramic. In addition, the recorded properties of APC 850 are measured at resonant frequency, which is much higher than the low frequencies tested experimentally. These indications, along with variability in manufacturing, can result in differences from theoretical values.

\subsubsection{Electromechanical Power Generation}

Five CLACS from each group were tested under dynamic compression over a shunting resistance sweep from $2 \mathrm{M} \Omega$ to $300 \mathrm{M} \Omega$. Figure 2.4 shows the average power generated as a function of structuring type and compliant layer thickness for a $2 \mathrm{~Hz}, 1000 \mathrm{~N}$ sine wave input. The maximum power in each group occurred at the same resistance of $77 \mathrm{M} \Omega$. Neither the addition of a compliant layer nor the dielectrophoretic structuring affected the source impedance of the CLACS. As expected, power production in each group significantly increased with an increase in frequency $(\mathrm{p}<0.10)$. The relationship was near linear with twice the power generated from 1 to $2 \mathrm{~Hz}$, three times the power from 1 to $3 \mathrm{~Hz}$, and 4.7 times the power from 1 
to $5 \mathrm{~Hz}$. These results were consistent across all structuring types, compliant layer thicknesses, resistances, and load levels tested.
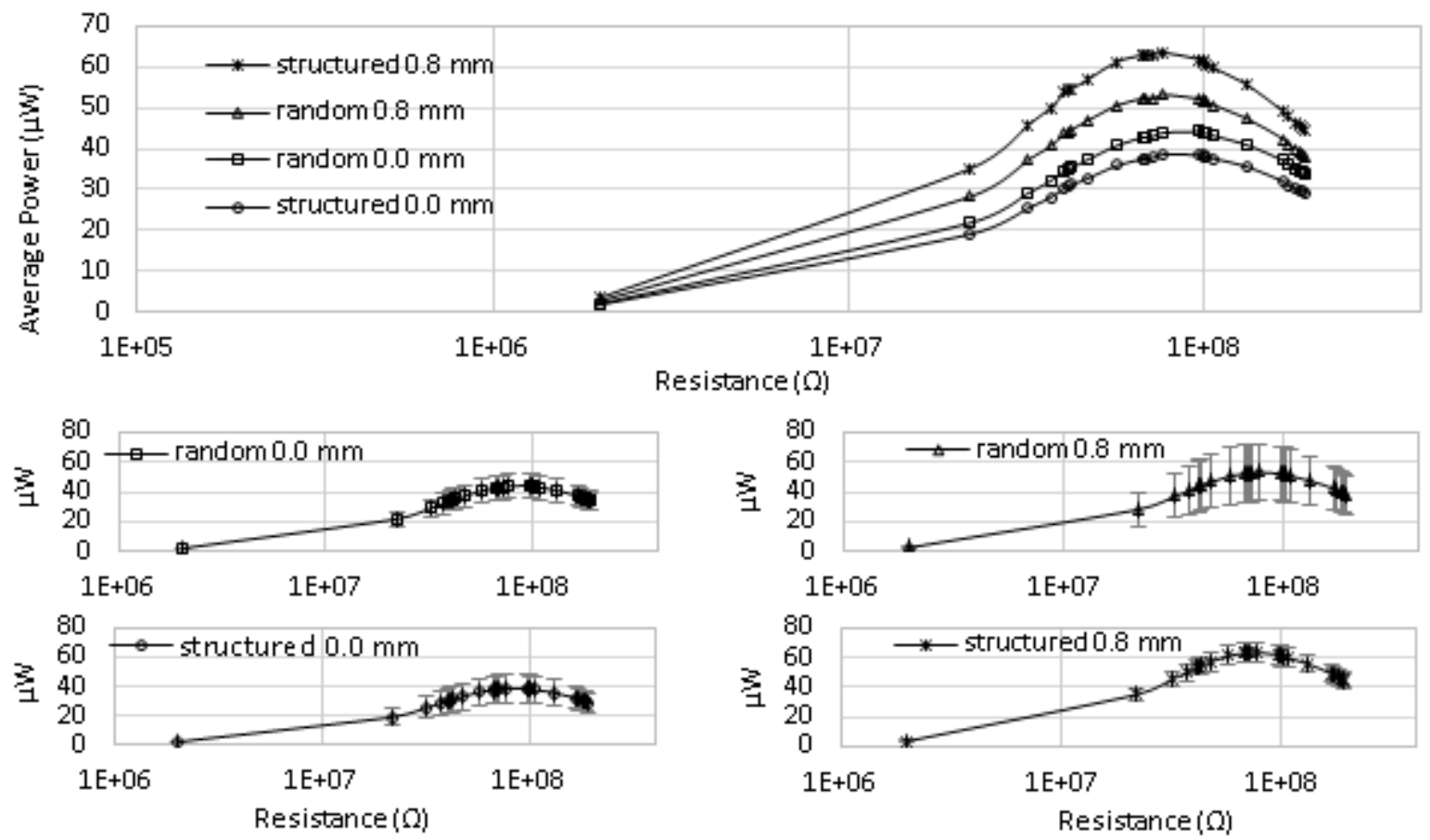

Figure 2.4: Average power output as a function of structuring type, compliant layer thickness, and resistance load of a 50\% VF composite stack when mechanically loaded at $1000 \mathrm{~N}$ and $2 \mathrm{~Hz}$.

Maximum power generated occurred at the highest load and frequency $(1000 \mathrm{~N}, 5 \mathrm{~Hz})$ for all groups and is displayed in Table 2.1. Both Figure 2.4 and Table 2.1 show the DEP structured, 0.8 mm compliant layer group producing the most power, with statistical significance between the $0.0 \mathrm{~mm}$ and $0.8 \mathrm{~mm}$ DEP structured groups $(\mathrm{p}<0.05)$. There was an average amplification of $19 \%$ between the $0.0 \mathrm{~mm}$ and $0.8 \mathrm{~mm}$ compliant layer random composite groups and $65 \%$ between the $0.0 \mathrm{~mm}$ and $0.8 \mathrm{~mm}$ compliant layer DEP structured groups. The average amplification caused by interdigitating compliant layers in the composite stacks correlates well with the theoretical model (26\% and 50\% amplification respectively). 
Table 2.1: Average peak power with respect to thickness and structuring type (1000 N, $5 \mathrm{~Hz}, 40.5 \mathrm{M} \Omega)$

\begin{tabular}{cc}
\hline Composite configuration & Average Maximum Power $(\boldsymbol{\mu W})$ \\
\hline random $0.0 \mathrm{~mm}$ & $105 \pm 19$ \\
\hline random $0.8 \mathrm{~mm}$ & $125 \pm 44$ \\
\hline structured $0.0 \mathrm{~mm}$ & $91 \pm 23$ \\
\hline structured $0.8 \mathrm{~mm}$ & $150 \pm 15$ \\
\hline
\end{tabular}

The average voltage for each group as a function of structuring type, compliant layer thickness, and frequency at $1000 \mathrm{~N}$ is presented in Figure 2.5. The nonlinearity of the voltagefrequency relationship can be observed in Figure 2.5 and suggests compliant layers are most effective at increasing voltage production at low frequencies. For 0-3 composites, the $0.8 \mathrm{~mm}$ group produced on average $10 \%$ more voltage compared to the $0.0 \mathrm{~mm}$ group. For $1-3$ composites, the $0.8 \mathrm{~mm}$ group produced on average $33 \%$ more voltage compared to the $0.0 \mathrm{~mm}$ group.

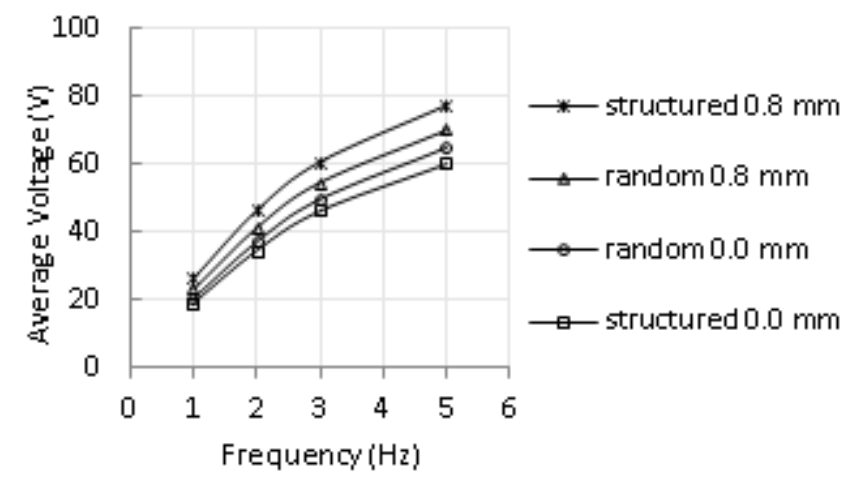

Figure 2.5: Average voltage with respect to structuring type, compliant layer thickness, and testing frequency for $50 \%$ VF composite CLACS. Representative voltage data presented at maximum power $(1000 \mathrm{~N}, 40.5 \mathrm{M} \Omega$ ). 


\subsection{Discussion}

This study was designed to investigate the effect of compliant layers, dielectrophoresis, and the combined effect on power generation and sensing ability of piezoelectric particle-composites at loads and frequencies observed in typical human $\operatorname{motion}^{17,18}$. However, other applications may benefit from these methods, such as high frequency, high strain, or any application where enhanced performance or increased toughness of a piezoelectric generator is desired ${ }^{19,20}$.

The combined theoretical model for DEP structuring and compliant layer amplification successfully modeled the power generation of CLACS made with thin particle composite discs. A slight increase in theoretical maximum power between the random and structured groups is observed (Figure 2.2). This agrees with the findings of Van den Ende et al. and is attributed to enhanced connectivity of particles from chain formation ${ }^{5,6,10}$. Khanbareh et al. report that DEP is less effective at high ceramic volume fractions due to overall enhanced connectivity ${ }^{7}$. Theoretical and experimental results agree with Khanbareh et al., showing similar power produced by 0-3 and 1-3 composite stacks without compliant layers ${ }^{7}$.

The results of this study agree with the findings of Krech et al, showing enhanced power production by using $0.8 \mathrm{~mm}$ compliant layers between thin piezoelectric composite discs in a stacked generator ${ }^{10}$. For all groups, the addition of a compliant layer enhanced the power production under compressive loads due to increased sensitivity to through thickness compression from positive in-plane strains ${ }^{14}$. The relationship between compliant layer thickness

and power production is expected to be linear, as seen in the results of Krech et al. ${ }^{14}$. The random and structured $0.8 \mathrm{~mm}$ CLACS were expected to perform equivalently; however, fiber-like chains formed during DEP were found to amplify the effect of the compliant layer. In a study by James et al., DEP structured composite achieved a higher flexural modulus than random 
composites due chain formation in the loading direction ${ }^{13}$. The driving factor in the shear lag theory for CLACS is the stiffness ratio (Equation 2.7) ${ }^{14}$. Therefore, an increase in stiffness rom DEP results in greater power amplification from compliant layers. Assuming DEP creates fully connected chains aligned in the thickness direction, the Halpin-Tsai model for continuous fibers in the thickness successfully modeled this increase in stiffness ${ }^{16}$. This showed good correlation with experimental results, and the effect of DEP structuring on power generation with $0.8 \mathrm{~mm}$ complaint layers can be observed by the slope in Figure 2.2. The average amplification of $65 \%$ from compliant layers for 1-3 groups matches the $61 \%$ amplification observed by Krech et al. ${ }^{10,14}$, while the $0-3$ groups only achieve $19 \%$ amplification from compliant layers.

When compared to bulk PZT materials, PZT composites of 50\% volume fractions experience a $98 \%$ reduction in piezoelectric properties ${ }^{7,21}$. This loss is due to the resulting connectivity of particles surrounded by matrix epoxy. The composite stacks in this study were designed to have the same volume of PZT $\left(157 \mathrm{~mm}^{3}\right)$, disc thickness, and surface area as the original CLACS study by Krech et al.. The average maximum power achieved by Krech et al. for $0.8 \mathrm{~mm}$ CLACS was $4883 \mu \mathrm{W}^{10}$. At equivalent loading conditions, the average maximum power of the 1-3 composite $0.8 \mathrm{~mm}$ CLACS was $150 \mu \mathrm{W}$. Therefore, with the same volume of PZT, only $3 \%$ of the maximum power was achieved. Even so, this power may be sufficient for MEMS that consume minimal power ${ }^{2}$.

Variation is introduced throughout the manufacturing process of PZT particle CLACS. These large variances resulted in a lack of statistical power to detect significant differences between most groups. The deviations in performance from sample to sample may result from sanding and shaping of each disc, porosity, stack alignment, poling, electrode thickness, compliant layer thickness, or overall height of the sample. Unlike other piezoelectric composite studies, however, 
this study presents successful methods of producing larger quantities of piezoelectric composite materials and presents electromechanical results.

Future work should be done to improve manufacturing methods and reduce variability. In addition, more volume fractions should be tested, along with additional thicknesses of compliant layers to identify ideal configurations of particle composite CLACS. The performance of custom shaped piezoelectric particle composites should also be investigated. This study focused on characterizing low frequency power production for MEMS, sensors, or medical devices; however, other work should be done to analyze the effect of DEP structuring and CLACS at higher frequencies.

\subsection{Conclusions}

This work presented an increase in power produced by piezoelectric composite generators by dielectrophoretic structuring of the ferroelectric ceramic particles inside a polymer matrix. In addition, this increase in power was amplified by the addition of compliant layers to form CLACS. The effect of combining DEP structured composite discs and compliant layers significantly increased power generation due to an increase in stiffness from chain formation. The theoretical model proposed in this study was successful in predicting the maximum power generated for both 0-3 and 1-3 particle composite CLACS. These methods provide further enhancement to power production and sensing capabilities of structured granular composites, making them more desirable because of their low cost manufacturing, tunable piezoelectric properties, shapeability, and mechanical stability ${ }^{7,20}$. 


\section{Acknowledgements}

This work was financially supported by the KU Research Go grant 00049907. The authors would like to thank the University of Kansas for their support of graduate researchers through assistantship funding.

\section{References}

1. Anton SR, Sodano HA. A review of power harvesting using piezoelectric materials (20032006). Smart Mater Struct. 2007;16:R1-R21.

2. Li H, Tian C, Deng ZD. Energy harvesting from low frequency applications using piezoelectric materials. Appl Phys Rev. 2014;1:041301.

3. Platt SR, Farritor S, Haider H. On low-frequency electric power generation with PZT ceramics. IEEEASME Trans Mechatron. 2005;10:240-52.

4. Goetzinger $\mathrm{N}$. The influence of stacked generators on source impedance of a piezoelectric spinal fusion implant [Internet] [Thesis]. University of Kansas; 2014 [cited 2019 Feb 25]. Available from: https://kuscholarworks.ku.edu/handle/1808/15925

5. Van den Ende DA, Van Kempen SE, Wu X, Groen WA, Randall CA, Van der Zwaag S. Dielectrophoretically structured piezoelectric composites with high aspect ratio piezoelectric particles inclusions. J Appl Phys. 2012;111:124107.

6. Van den Ende DA, Bory BF, Groen WA, Van der Zwaag S. Improving the d33 and g33 properties of 0-3 piezoelectric composites by dielectrophoresis. J Appl Phys. 2010;107:024107.

7. Khanbareh H, Van der Zwaag S, Groen WA. Effect of dielectrophoretic structuring on piezoelectric and pyroelectric properties of lead titanate-epoxy composites. Smart Mater Struct. 2014;23:105030.

8. Newnham RE. Connectivity and Piezoelectric-Pyroelectric Composites. Mater Res Bull. 1978;13:525-36.

9. Yamada T, Ueda T, Kitayama T. Piezoelectricity of a high-content lead zirconate titanate/polymer composite. J Appl Phys. 1982;53:4328-32.

10. Krech ED, Cadel ES, Barrett RM, Friis EA. Effect of compliant layers within piezoelectric composites on power generation providing electrical stimulation in low frequency applications. J Mech Behav Biomed Mater. 2018;88:340-5. 
11. Groen P, Holterman. An Introduction to Piezoelectric Materials and Components. Stichting Applied Piezo; 2012.

12. Pethig R. Review Article-Dielectrophoresis: Status of the theory, technology, and applications. Biomicrofluidics. 2010;4:022811.

13. James NK, Ende D van den, Lafont U, Van der Zwaag S, Groen WA. Piezoelectric and mechanical properties of structured PZT-epoxy composites. J Mater Res. 2013;28:635-41.

14. Krech ED, Barrett RM, Cadel ES, Friis EA. Power Generation Amplification and Stack Toughening via Compliant Layer Interdigitation. In American Society of Mechanical Engineers; 2018.

15. Bowen C. Dielectric properties of dielectrophoretically assembled particulate-polymer composites. JMR. 2011;13:205-10.

16. Halpin JC. Stiffness and Expansion Estimates for Oriented Short Fiber Composites. J Compos Mater. 1969;3:732-4.

17. Pachi A, Ji T. Frequency and velocity of people walking. Struct Eng. 2005;83.

18. Lutz F, Mastel R, Runge M, Stief F, Schmidt A, Meurer A, et al. Calculation of muscle forces during normal gait under consideration of femoral bending moments. Med Eng Phys. 2016;38:1008-15.

19. Van den Ende DA, Groen WA, Van der Zwaag S. Robust piezoelectric composites for energy harvesting in high-strain environments. J Intell Mater Syst Struct. 2013;24:2262-9.

20. Van den Ende DA, Wiel HJ van de, Groen WA, Van der Zwaag S. Direct strain energy harvesting in automobile tires using piezoelectric PZT-polymer composites. Smart Mater Struct. 2011;21:015011.

21. Thongsanitgarn $P$, Watcharapasorn A, Jiansirisomboon S. Electrical and mechanical properties of pzt/pvdf 0-3 composites. Surf Rev Lett. 2010;17:1-7. 


\section{Chapter 3: Detailed Methods}

\subsection{Experimental Setup}

The entire system setup, DEP mold schematic, and poling schematic used in this study are displayed in Figures 3.1, 3.2 and 3.3. The setup in Figure 3.1 was used for both dielectrophoresis, done in the early stages of manufacturing, and poling, which is done in the middle stages of manufacturing. The hot plate is utilized in both processes, which should never be performed at the same time, so it is simply moved for use depending on the current process. The function generator produces a sine wave input of $2 \mathrm{~V} / \mathrm{mm}$ which is amplified 1000 times by the Trek AC amplifier to deliver $2 \mathrm{kV} / \mathrm{mm}$ at $2 \mathrm{~Hz}$ for DEP. The Trek amplifier has an output

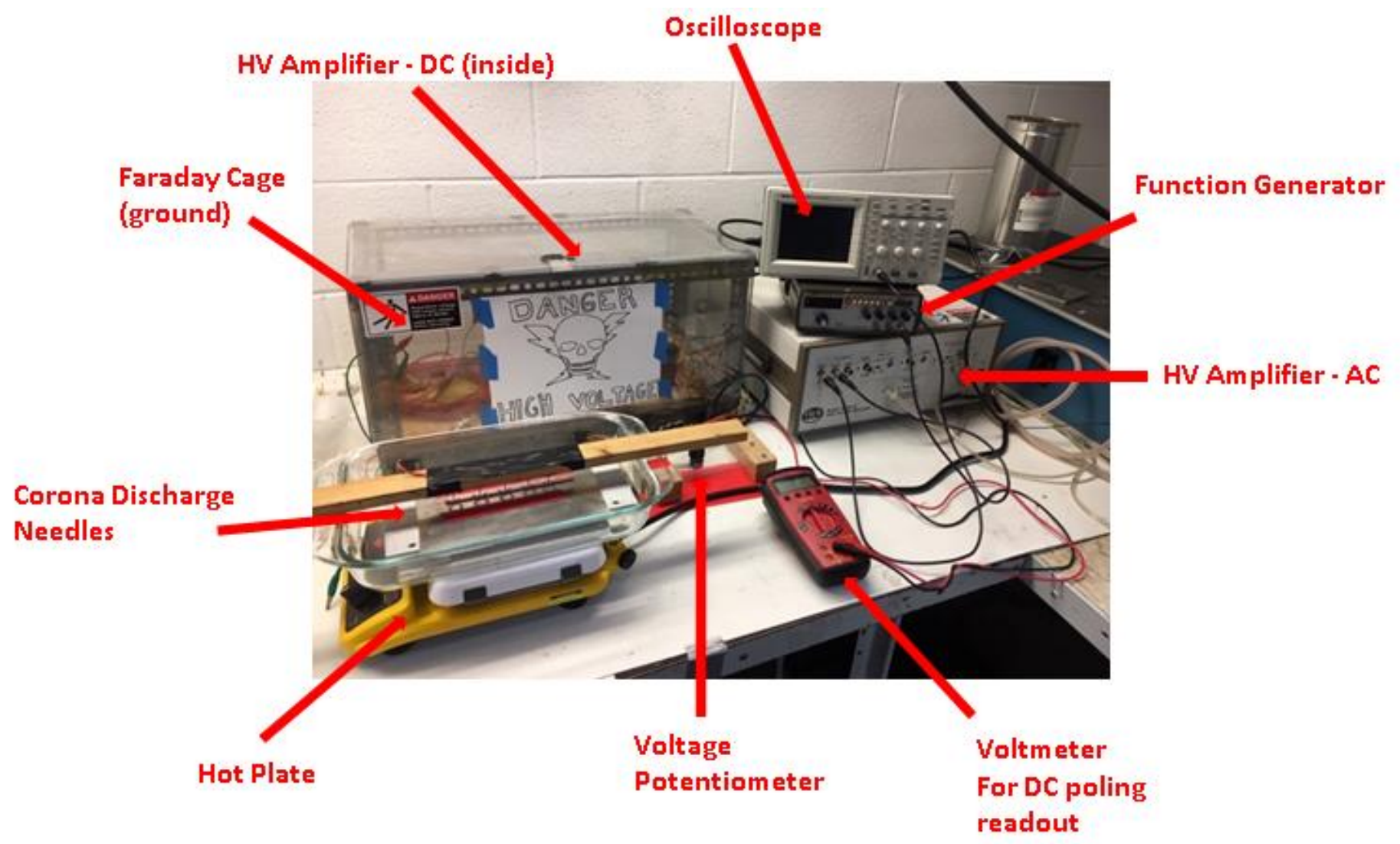

Figure 3.1: Entire experimental manufacturing setup for poling (left) and dielectrophoresis (right). 
cable to send the $2 \mathrm{~V} / \mathrm{mm}$ input voltage to the oscilloscope, to verify the amplified current is being delivered properly and is not shorting across the electrodes. Detailed procedures for both DEP and poling can be found in Appendix D.

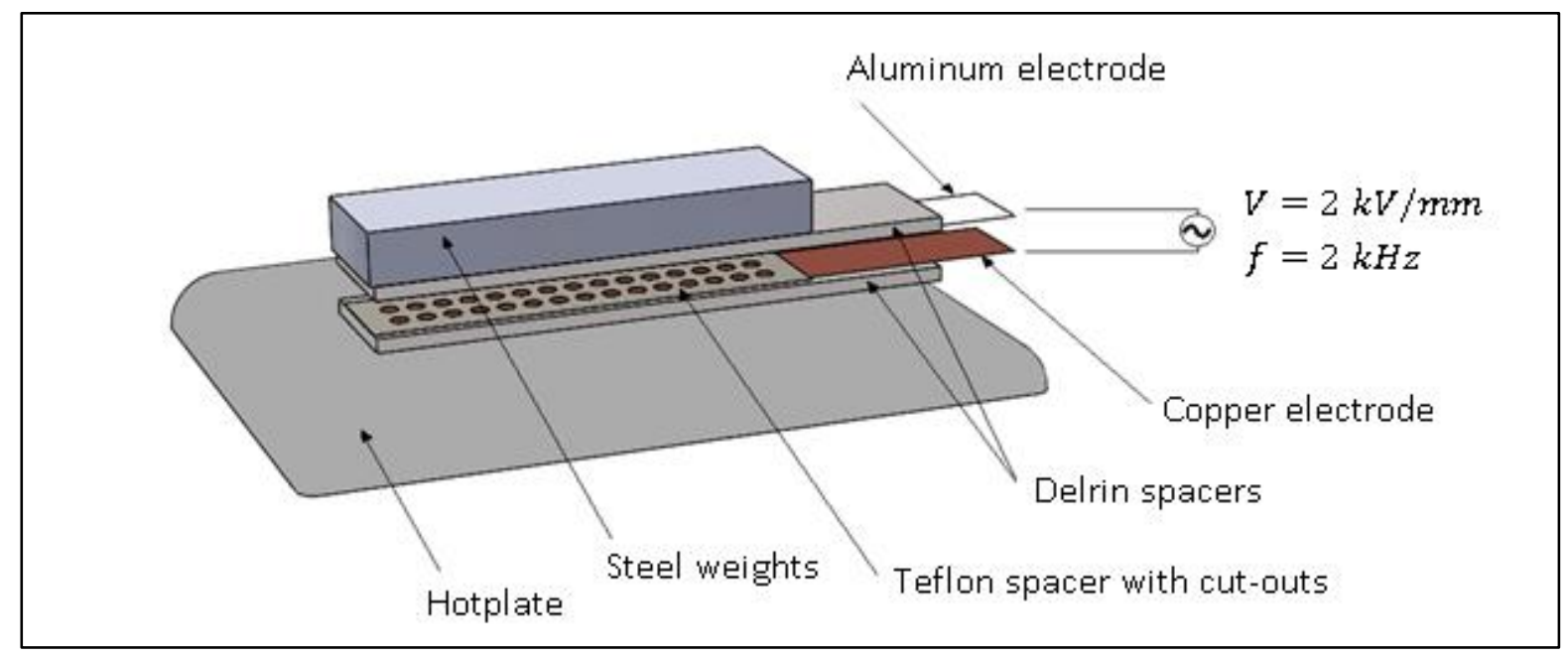

Figure 3.2: Schematic of mold setup for dielectrophoresis processing.

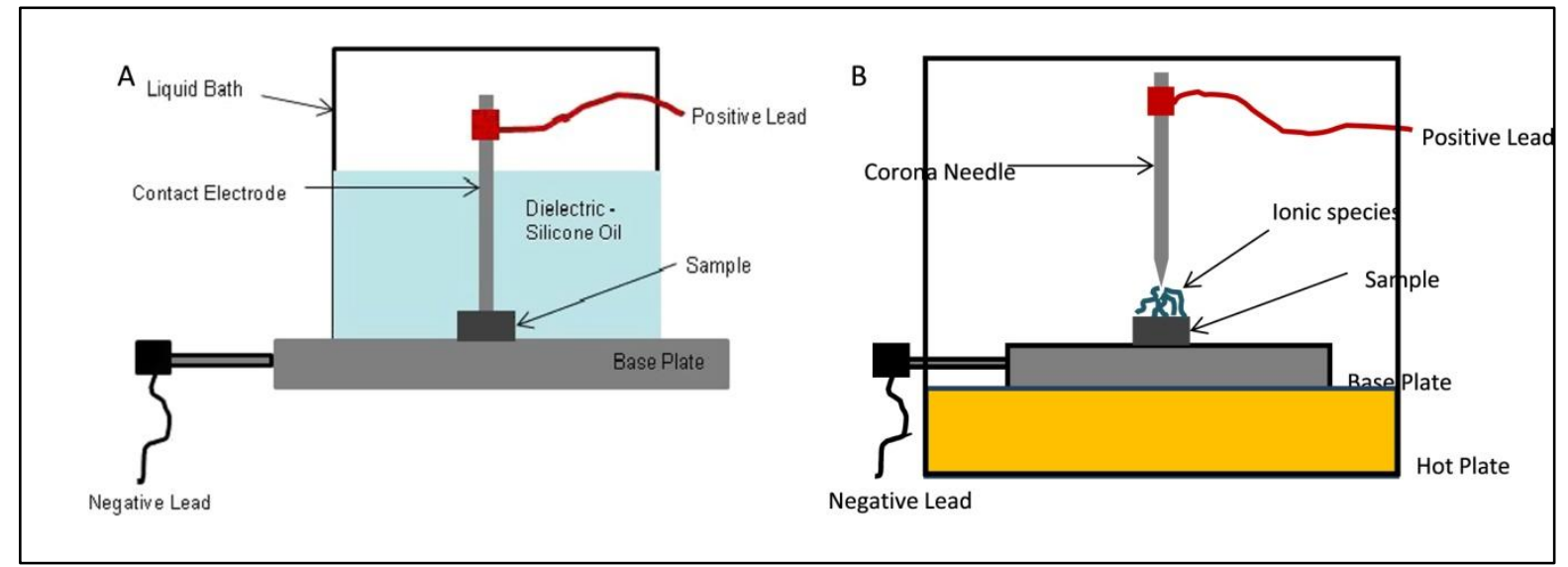

Figure 3.3: Schematic of setup of both the parallel plate-contact method (A) and the corona discharge poling method (B). (C) IOP Publishing. Reproduced with permission. All rights reserved. 


\subsection{Manufacturing PZT particle composites}

Lead Zirconate Titanate (PZT) powder, a Navy Type II, PZT-5 material was selected for this study (American Piezo Ceramics, Mill Hall, PA). The powder arrives from the supplier spray dried with a $1 \%$ polyvinyl alcohol binder resulting in free flowing agglomerates of 50-150 micrometers in diameter. Each sphere is made up of 0.5 micrometer PZT crystallites. In $100 \%$ PZT ceramic manufacturing, the binder holds the PZT crystallites into the correct shape and is burned off during sintering, resulting in a dense PZT ceramic material. In order to utilize these powder materials in composites, the binder must be sintered off to disaggregate the particles to individual crystallites and improve connectivity. The powder in this study was sintered at $700{ }^{\circ} \mathrm{C}$ for 1 hour. Then the sintered powder was then ground with a mortar and pestle for 5 minutes to disaggregate the agglomerate spheres.

A two component, medical grade epoxy (EPO-TEK® 301, Epoxy Technology, Billerica, MA) was used to form the composite slurry. After combining the two part epoxy, particles were added to achieve a 50\% volume fraction (VF) of ceramic content, and mixed for 15 minutes with a spatula. See Appendix A.1 for the MATLAB code used to obtain the required mass of each epoxy component and sintered powder in order to achieve the desired VF. A 50\% VF was utilized in this study in order to maximize connectivity and power generation; however, it is known that DEP is more effective at low volume fractions ${ }^{22,23,48}$. It is important to note, that the EPO-TEK 301 epoxy is slightly conductive before curing. Therefore, there were often issues performing DEP at lower volume fractions due to shorting across the electrodes. 
Once successfully mixed, the composite slurry, or putty, was then placed into a $0.4 \mathrm{~mm}$ thick Teflon mold, consisting of 30, $10 \mathrm{~mm}$ cut-outs (Figure 3.4). Once filled, the aluminum electrode is placed on top, and the mold assembled into the setup for composite curing and DEP shown in Figure 3.2. Directly after the setup was placed on the hotplate, a function generator (B\&K

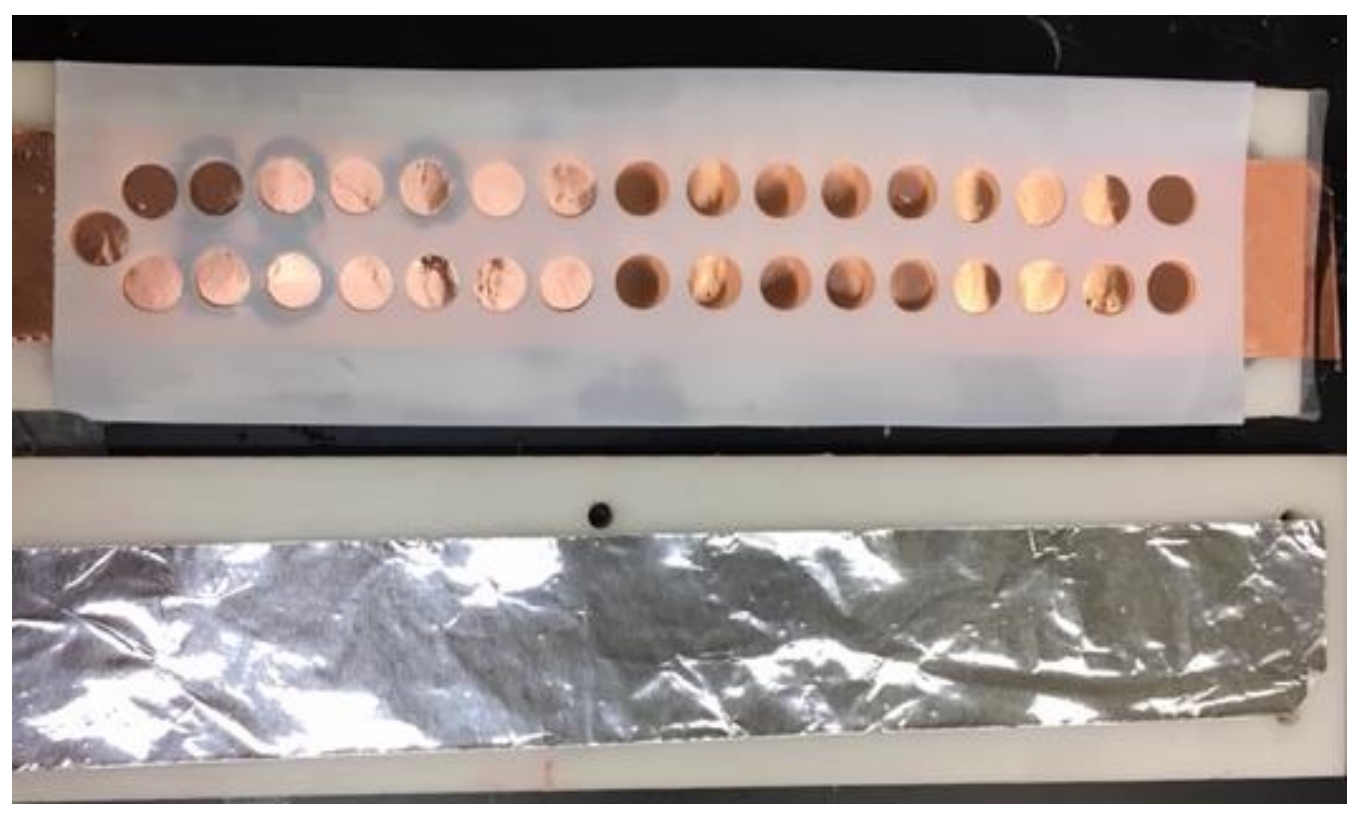

Figure 3.4: Teflon mold and electrodes for dielectrophoresis.

Precision, Yorba Linda, CA) coupled to a high voltage amplifier (10/10B-HS, TREK, Lockport, NY) was used to structure the particles in the uncured composite by means of dielectrophoresis. The electric field of $2 \mathrm{kV} \mathrm{mm}^{-1}$ and frequency of $2 \mathrm{kHz}$ was applied across the electrodes for 3 hours at $65^{\circ} \mathrm{C}$ then applied for 1 hour while cooling to room temperature. These testing parameters were pulled from the work of Van den Ende et al., and were chosen to best fit the PZT type and thickness that was used in this study. The non-structured, or randomly dispersed, samples were manufactured in the same fashion without applying an electric field by curing at room temperature for 24 hours. 
After DEP processing and curing, the discs were polished with sandpaper to $0.4 \pm 0.03 \mathrm{~mm}$ thickness to remove the top epoxy layer. Even though the Teflon mold was only $0.4 \mathrm{~mm}$ thick, the cured discs were closer to $0.6 \mathrm{~mm}$ thick due to incompressibility and overfilling. It is recommended to use a slightly thinner Teflon sheet to conserve material and reduce the time of post processing. In addition, a dremel with a fine sanding attachment was used to clean the edges and shape the discs to achieve a $10 \mathrm{~mm} \pm 0.03 \mathrm{~mm}$ diameter. However, this made it difficult to achieve perfectly circular discs.

After cleaning and drying the discs, a copper electrode of $9.2 \mathrm{~mm}$ diameter, $25.4 \mu \mathrm{m}$ thickness was sprayed onto one side using copper conductive spray (843AR Super Shield ${ }^{\mathrm{TM}}$, MG Chemicals, Surrey, BC). The setup for spraying is shown in Figure 12. The discs are placed close

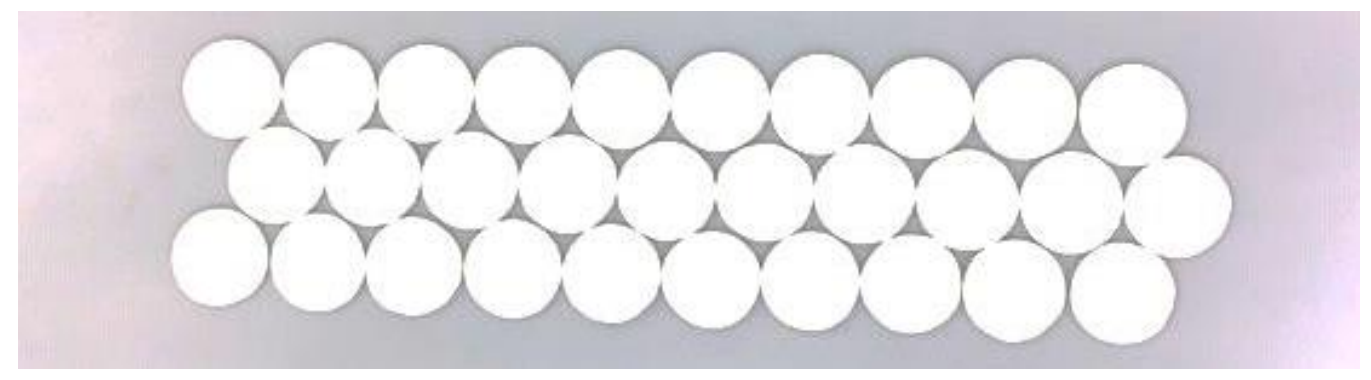

Figure 3.5: Setup for 30 discs to be sprayed for electrode application

together to conserve the copper spray; however, this required further processing to remove the electrode from all edges with a scalpel. 3D printed stencils were investigated in order to allow consistent diameters of electrodes; however, the spray builds up on the stencil quickly, more spray is wasted, and there is still a need for scraping excess and edges. Ultimately, it was less expensive to use the method shown in Figure 3.5, but better methods of electrode placement could be further investigated. 
Two different poling methods were considered in this work. The original method of parallel plate contact poling was investigated for the poling of PZT composites. However; unlike PZT ceramics, the composites require much higher voltages for poling due to the difference in dielectric properties of the particles and the surrounding epoxy $y^{51,53}$. These high voltages can cause dielectric breakdown of the composite and create a short across the electrodes, especially when there are pinholes, bubbles, or other weak points ${ }^{51,53}$. This was observed in the early testing of this study whenever poling was attempted above $4 \mathrm{kV}$.

In order to achieve poling voltages of $15 \mathrm{kV} \mathrm{mm}^{-1}$, the corona discharge method was implemented. A corona poling rig was built by assembling $10 \mathrm{~T}$-pins in a wood board with the needle tips placed $7.5 \mathrm{~mm}$ above the discs surfaces. The 10 needles were all soldered, connecting

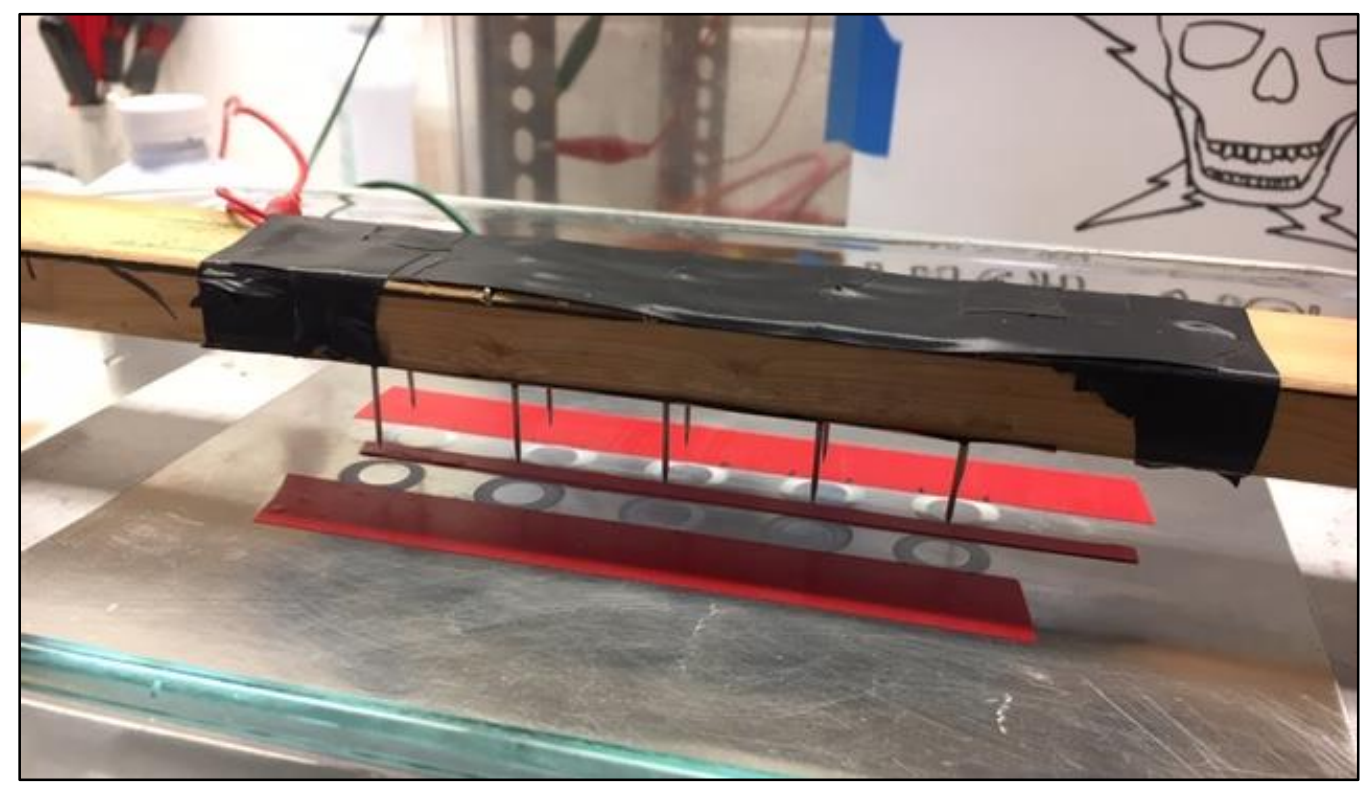

Figure 3.6: Corona discharge setup with 10 needle points connected in parallel with the aluminum plate as ground.

them electrically, and the aluminum plate in contact with the electroded disc faces was connected to ground. Poling was carried out with $6 \mathrm{kV}(15 \mathrm{kV} / \mathrm{mm})$ of DC across 10 discs for 30 minutes at $100{ }^{\circ} \mathrm{C}$. The temperature was achieved by placing the setup on top of a hotplate. The discs were 
then cooled to room temperature in the presence of the poling field for 30 minutes and stored with the ends shorted to remove any trapped charges form polarization or impurities. Lastly, the second electrode was sprayed onto each disc using the same method stated earlier. Poling success was verified by pyroelectric analysis of each disc. To carry out pyroelectric testing, each disc was placed on a conductive surface. Then the positive lead from the voltmeter is placed on the top of the disc, the negative is placed on the conductive surface, and the tester puffs a short breath directly on the disc. The change in temperature causes a change in voltage that can be measured. This test is used to determine the positive electrode, which should be marked once tested, and gauge the success of poling by checking similarity of voltage produced by each disc.

\subsection{CLACS Manufacturing}

Compliant Layer Adaptive Composite Stacks (CLACS) were manufactured using two different compliant layer thicknesses for both DEP structured (1-3) discs and random (0-3) discs ( $n=5$ in each group, $N=20$ ). The compliant layer thicknesses were chosen to match the original CLACS study by Krech et al.; however, only the $2 \mathrm{x}$ thickness $(0.8 \mathrm{~mm})$ was chosen because it produced the largest amplification. Other thicknesses could be tested, but both the theoretical and experimental results match the relationship presented by Krech et al., deeming it unnecessary.

The manufacturing methods of CLACS were produced by Ember Krech, a PhD graduate candidate in the Spine Biomechanics Laboratory at the University of Kansas. Very detailed methods for manufacturing CLACS are presented in the Thesis submitted by Kyle Coates at the University of Kansas (Coates, 2018, p. 108-114) ${ }^{54}$. These methods are valid for both PZT ceramic or composite discs and were implemented in this work. 


\subsection{Developing the Theoretical Model}

A significant portion of this work was focused on developing a theoretical model to predict the power production capabilities and power amplification caused by implementing CLACS. This was approached by characterizing the properties of the particle composites, determining mechanical properties, implementing current models for Dielectrophoretic structuring, and lastly implementing the shear lag model for compliant layers.

\subsubsection{PZT-Epoxy Connectivity and Stiffness}

In order to determine the stiffness of the particle composite materials, the Halpin-Tsai equations ${ }^{55,56}$ were implemented for both randomly distributed particles within a polymer matrix and aligned continuous fibers (or chains of particles structured by dielectrophoresis). As stated previously, Newnham et al. defines patterns of connectivity for two phase composites. One pattern is 0-3 connectivity, or an assumed equal, random distribution of ceramic phase throughout the fully connected polymer phase. The second type is $1-3$, or aligned fibers of ceramic phase within the polymer phase ${ }^{16}$. Van den Ende et al. achieved quasi 1-3 connectivity by implementing dielectrophoresis (DEP) to structure the particles into chains ${ }^{22,48}$. However, there is not much discussion on how this affects stiffness, which is necessary for calculating the strain in the piezoelectric material. For random, 0-3 composites the stiffness was determined by the Halpin-Tsai equations:

$$
E_{\text {Comp }}=E_{M}\left(\frac{1+\zeta N \Phi}{1-N \Phi}\right)
$$

where, 


$$
N=\frac{\left(\frac{E_{c o m p}}{E_{M}}\right)-1}{\left(\frac{E_{c o m p}}{E_{M}}\right)+\zeta}
$$

where,

$$
\zeta=2+40 \Phi^{10}
$$

where $\zeta$ is the factor used to describe the influence of geometry, $\Phi$ is the volume fraction, and the subscripts comp and $M$ refer to composite and matrix properties respectively. For the randomly dispersed spherical particles, the appropriate $\zeta$ value was used. However, in order to best characterize the DEP structured composites, the equation for aligned continuous fibers was used, since the chain structuring will produce fiber-like reinforcement in the thickness direction. The modulus for such 1-3 composites was determined by:

$$
E_{\text {comp }}=\Phi E_{f}+(1-\Phi) E_{M}
$$

\subsubsection{Piezoelectric Characterization}

The analytical model used to determine the piezoelectric properties of $0-3$ or random composites is proposed by Yamada et al. ${ }^{57}$. First, the piezoelectric particles must be characterized using a set of equations coined "Maxwell's equations" that define the electrical potentials outside and inside an ellipsoid particle $(a, b, c)^{57}$. From those equations, the depolarization factor, $m$, is determined by:

$$
m=\int_{0}^{\infty} \frac{d u}{\left(a^{2}+u\right) \beta_{(u)}}
$$

where,

$$
\beta_{(u)}=\frac{\left[\left(a^{2}+u\right)\left(b^{2}+u\right)\left(c^{2}+u\right)\right]^{1 / 2}}{2 \pi a b c}
$$


For simplicity, and lack of advanced equipment, the particles were assumed to be sphere $(a=b=c)$. The composite is then assumed to have random distribution of these particles within a polymer matrix wherein the dielectric constant, $\varepsilon$, and piezoelectric charge constant, $d_{33}$, in the poling direction are determined by:

$$
K_{\text {random }}=\varepsilon_{M}\left(1+\frac{\eta \Phi\left(\varepsilon_{m}-\varepsilon_{c}\right)}{\eta \varepsilon_{m}+\left(\varepsilon_{c}-\varepsilon_{m}\right)(1-\Phi)}\right)
$$

where $\eta=4 \pi / m$ is the inverse of the depolarization factor, or a parameter attributed to the shape of the particle. The piezoelectric charge constant can then be obtained by:

$$
d_{33_{\text {random }}}=\frac{\Phi \alpha \eta K_{\text {random }} d_{33_{c}}}{\eta K_{\text {random }}+\varepsilon_{c}-K_{\text {random }}}
$$

where $K$ is the dielectric constant, $\eta$ is the inverse of the depolarization factor, $\Phi$ is the volume fraction of the ceramic phase, $\alpha$ is the poling ratio of ceramic particles and other parameters introduced in Equation 3.7, and the subscripts $m, c$, and random refer to the polymer matrix, ceramic, and 0-3 properties respectively. The best fit of experimental data to the model predictions was obtained using a value of 1.2 for $\alpha$.

The Bowen model was developed from the Yamada model and is used to determine piezoelectric properties of the structured, or quasi 1-3 composites ${ }^{58}$. As mentioned earlier, it is assumed that DEP has formed chains or fibrils of particles in line with the electric field surrounded by matrix epoxy. The equation for the dielectric constant for such 1-3 composites can be determined by:

$$
\mathrm{K}_{\text {structured }}=\Phi_{\mathrm{c}}\left(\frac{\mathrm{R} \varepsilon_{\mathrm{c}} \varepsilon_{\mathrm{m}}}{\varepsilon_{\mathrm{c}}+\mathrm{R} \varepsilon_{\mathrm{m}}}\right)+\left(1-\Phi_{\mathrm{c}}\right) \varepsilon_{\mathrm{m}}
$$


where $R$ is the ratio of average particle size to the effective interparticle distance. A value of 6.2 for $\mathrm{R}$ was obtained in order to achieve best fit of experimental data to the Bowen model. Beyond dielectric characterization, Van den Ende et al. developed an extension to Bowen's model in order to characterize the $d_{33}$ of structured composites. This was achieved by modeling the particle chains as two capacitors in series electrically and two springs in series mechanically ${ }^{23}$ and is derived by:

$$
\mathrm{d}_{33_{\text {structured }}}=\frac{(1+\mathrm{R})^{2} \varepsilon_{\mathrm{m}} \Phi_{\mathrm{c}} \mathrm{d}_{33} \mathrm{Y}_{\mathrm{c}}}{\varepsilon_{\mathrm{c}}+\mathrm{R} \varepsilon_{\mathrm{m}}\left[\left(1+\mathrm{R} \Phi_{\mathrm{c}}\right) \mathrm{Y}_{\mathrm{c}}+\left(1-\Phi_{\mathrm{c}}\right) \mathrm{RY} Y_{\mathrm{m}}\right.}
$$

where $Y$ is the elastic modulus and $\varepsilon$ is the dielectric constant of either the PZT or matrix epoxy phase, not the overall composite. The remaining characterization was determined utilizing developed piezoelectric theory from American Piezoelectric Ceramics (APC).

The theory developed by APC provides us with a theoretical electromechanical coupling factor $\left(k_{33}\right)$, or an indicator of effectiveness with which a piezoelectric material converts mechanical energy into electrical energy ${ }^{44}$. The $k_{33}$ can be calculated for both random and structured composites by implementing the Halpin-Tsai, Yamada, Bowen, Van den Ende, and APC models and is determined by:

$$
\mathrm{k}_{33}=\frac{\mathrm{d}_{33}}{\sqrt{s_{33}^{E} \varepsilon_{33}^{T}}}
$$

where $s_{33}$ is the elastic compliance, relating strain developed to stress applied, and is given by:

$$
s_{33}^{E}=\frac{1}{Y_{33}^{E}}
$$


where $\varepsilon_{33}$ is the permittivity of the PZT and $Y_{33}$ is the modulus derived by the Halpin-Tsai equations. The permittivity can now be determined for both 0-3 and 1-3 composites using the respective dielectric constant by:

$$
\varepsilon_{33}^{T}=\mathrm{K}^{\mathrm{T}} \varepsilon_{0}
$$

where $\varepsilon_{0}$ is the permittivity of free space $\left(8.85 \times 10^{-12} \mathrm{farad} / \mathrm{m}\right)$ and $\mathrm{K}^{\mathrm{T}}$ is the dielectric constant derived by either the Yamada or Van den Ende model. Now, with all necessary piezoelectric properties determined for the composites, baseline power generation can be determined.

\subsubsection{Power Prediction}

To predict the effect of the interdigitated compliant layers, the previously stated models were combined with the CLACS model by Krech et al. This model states the amplification from compliant layers is due to increases in positive in-plane strains caused by compression in the thickness direction ${ }^{59}$. The power was successfully modeled by implementing a shear lag

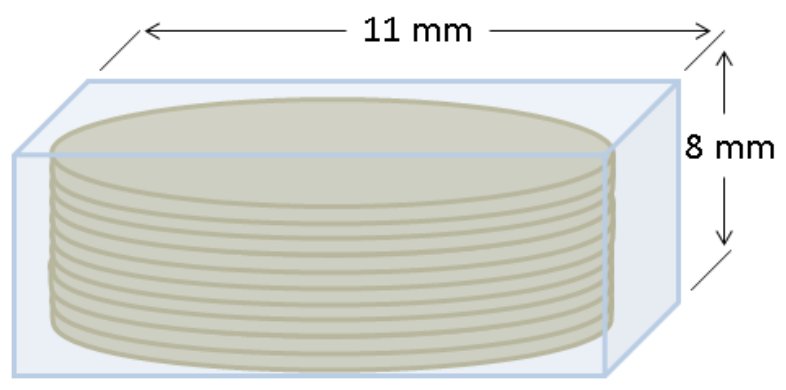

Figure 3.7: Piezoelectric particle stack without a compliant layer (11 mm x $11 \mathrm{~mm}$ x $8 \mathrm{~mm}$ ) 
structural mechanics model. It is assumed that the discs are all perfectly bonded to each other and only ideal compression is delivered in loading. The baseline power from a piezoelectric stack without compliant layers $\left(P_{0}\right)$ can be modeled as a function of work $(W)$, frequency $(f)$, and electromechanical coupling factor $\left(k_{33}\right)$ by:

$$
\mathrm{P}_{0}=\mathrm{W} * \mathrm{f} * \mathrm{k}_{33}^{2}
$$

where work $(W)$ is the product of the load and the change in thickness in the loading direction and $k_{33}$ is the electromechanical coupling factor determined with Equation 3.11. Now, by combining the results from previous models with the shear lag model by Krech et al., the power amplification due to the addition of compliant layers in piezoelectric stacks is given by the equation:

$$
\mathrm{AF}=\frac{\mathrm{P}}{\mathrm{P}_{0}} \sim 1+\left|\frac{\mathrm{d}_{31}}{\mathrm{~d}_{33}}\right| \mathrm{c} \sqrt{\left(\frac{\mathrm{t}_{\mathrm{cl}}}{2 \mathrm{t}_{\text {composite }}}\right)\left(\frac{\mathrm{E}_{\text {composite }}}{\mathrm{E}_{\mathrm{CL}}}\right)}
$$

where $t$ is thickness, $E$ is the modulus, c is the contiguity factor, and $d$ is the respective charge constant. The $E, \mathrm{~d}_{33}$, and $\mathrm{d}_{31}$ are obtained from the previous models for random and structured composites. The contiguity factor was determined to be 0.62 in order to refine the amplification factor and account for variability in manufacturing ${ }^{59}$. Equations 3.14 and 3.15 can be combined to predict the electromechanical coupling factor of the composite, resulting in the equation:

$$
\mathrm{k}_{33}=\sqrt{\mathrm{k}_{33_{0}}{ }^{2}\left(1+\left|\frac{\mathrm{d}_{31}}{\mathrm{~d}_{33}}\right| \mathrm{c} \sqrt{\left(\frac{\mathrm{t}_{\mathrm{cl}}}{2 \mathrm{t}_{\text {composite }}}\right)\left(\frac{\mathrm{E}_{\text {composite }}}{\mathrm{E}_{\mathrm{CL}}}\right)}\right)}
$$


Figure 3.8 shows the experimental results for maximum power conditions, and how they compare to the theoretical model predictions. Detailed discussion of experimental and theoretical results can be found in Chapter 2 .
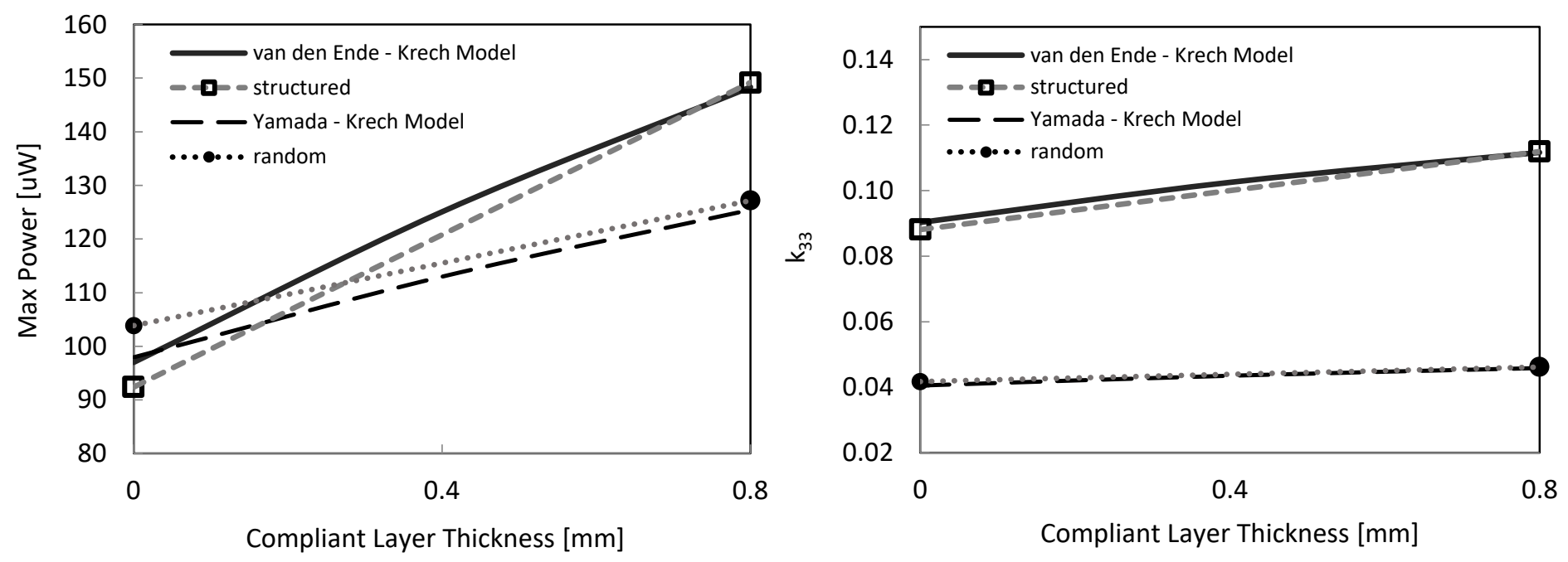

Figure 3.8: Maximum power (left) and $\mathrm{k}_{33}$ (right) theoretical predictions vs. experimental results under mechanical loading $(1000 \mathrm{~N}, 5 \mathrm{~Hz}, 5 \mathrm{M} \Omega)$ 


\section{Chapter 4 : Conclusions and Future Work}

The addition of a compliant layer between thin PZT particle composite discs to form CLACS has shown improved efficiency of power generating potential in mechanical loading. The combined theoretical model for piezoelectric particle composite theory and shear lag theory for CLACS was successful in predicting $\mathrm{k}_{33}$ and maximum power for all combinations of structuring type and compliant layer thickness. It is assumed that the formation of chains during DEP increases the composite stiffness, similar to fiber reinforced composites. The stiffness ratio is the driving factor of the shear lag theory, thereby amplifying the effect of compliant layers on power generation.

Some limitations to this study were the small sample size ( $n=5$ per group), especially with the large standard deviations observed. This reduced the statistical power of the study and thereby decreased the ability to detect significant differences between most groups; however, the success of the theoretical model supports the experimental results. Variation is introduced at many places throughout the manufacturing process of PZT particle CLACS. The main source of variance is most likely from porosity in the composite caused by air bubbles introduced during the mixing process. Van den Ende et al. and other particle composite researchers have access to vacuum chambers that remove air from the slurry before curing ${ }^{23}$. With stiffness and connectivity being crucial properties for performance, the presence of air bubbles can be an issue; however, the low cost methods used in this study are viable.

Future work could investigate improved manufacturing methods to reduce variability in performance. In addition, more volume fractions and additional thicknesses of compliant layers could be tested to identify ideal configurations of particle composite CLACS. The investigation of piezoelectric composites in femoral intramedullary nail was initiated, due to their ability to be 
manufactured into rings. See appendix D for the initial development and potential future applications of custom shaped composites. However, the present study was carried out first in order to determine the relationship between PZT composite discs and compliant layers in CLACS. The study also sought to develop a theoretical model to understand this relationship. Future work could build off of this study to investigate particle composites for the intramedullary nail and develop a theoretical model for ring shaped composite CLACS. 


\section{References}

1. Mills LA, Aitken SA, Simpson AHRW. The risk of non-union per fracture: current myths and revised figures from a population of over 4 million adults. Acta Orthop. 2017;88:434-9.

2. Giannoudis PV, MacDonald DA, Matthews SJ, Smith RM, Furlong AJ, De Boer P. Nonunion of the femoral diaphysis. J Bone Joint Surg Br. 2000;82-B:655-8.

3. Copuroglu C, Calori GM, Giannoudis PV. Fracture non-union: Who is at risk? Injury. 2013;44:1379-82.

4. Adams CI, Keating JF, Court-Brown CM. Cigarette smoking and open tibial fractures. Injury. 2001;32:61-5.

5. Calori GM, Mazza EL, Mazzola S, Colombo A, Giardina F, Romanò F, et al. Non-unions. Clin Cases Miner Bone Metab. 2017;14:186-8.

6. Rupp M, Biehl C, Budak M, Thormann U, Heiss C, Alt V. Diaphyseal long bone nonunions types, aetiology, economics, and treatment recommendations. Int Orthop. 2018;42:247-58.

7. Platt SR, Farritor S, Haider H. On low-frequency electric power generation with PZT ceramics. IEEEASME Trans Mechatron. 2005;10:240-52.

8. Frölke JPM, Patka P. Definition and classification of fracture non-unions. Injury. 2007;38:S19-22.

9. Hulth A. Current concepts of fracture healing. Clin Orthop. 1989;265-84.

10. Antonova E, Le TK, Burge R, Mershon J. Tibia shaft fractures: costly burden of nonunions. BMC Musculoskelet Disord. 2013;14:42.

11. Fukada E, Yasuda I. On the Piezoelectric Effect of Bone. J Phys Soc Jpn. 1957;12:1158-62.

12. Shybut G, Donley B. OsteoGen Surgically Implanted Bone Growth Stimulator Surgical Technique.

13. Toth JM, Seim HB, Schwardt JD, Humphrey WB, Wallskog JA, Turner AS. Direct current electrical stimulation increases the fusion rate of spinal fusion cages. Spine. 2000;25:2580-7.

14. Victoria G, Petrisor B, Drew B, Dick D. Bone stimulation for fracture healing: What's all the fuss? Indian J Orthop. 2009;43:117.

15. Mcdowell CS. Implanted bone stimulator and prosthesis system and method of enhancing bone growth [Internet]. 6143035, 2000. Available from: frolk

16. Newnham RE. Connectivity and Piezoelectric-Pyroelectric Composites. Mater Res Bull. 1978;13:525-36. 
17. Newnham RE, Bowen LJ, Klicker KA, Cross LE. Composite piezoelectric transducers. Mater Des. 1980;2:93-106.

18. Goetzinger NC, Tobaben EJ, Domann JP, Arnold PM, Friis EA. Composite piezoelectric spinal fusion implant: Effects of stacked generators. J Biomed Mater Res B Appl Biomater. 2016;104:158-64.

19. Krech ED, Cadel ES, Barrett RM, Friis EA. Effect of compliant layers within piezoelectric composites on power generation providing electrical stimulation in low frequency applications. J Mech Behav Biomed Mater. 2018;88:340-5.

20. Holterman J, Groen P. An Introduction to Piezoelectric Materials and Applications. Stichting Applied Piezo; 2013. 307 p.

21. Pethig R. Review Article-Dielectrophoresis: Status of the theory, technology, and applications. Biomicrofluidics. 2010;4:022811.

22. Khanbareh H, Van der Zwaag S, Groen WA. Effect of dielectrophoretic structuring on piezoelectric and pyroelectric properties of lead titanate-epoxy composites. Smart Mater Struct. 2014;23:105030.

23. Van den Ende DA, Bory BF, Groen WA, Van der Zwaag S. Improving the d33 and g33 properties of 0-3 piezoelectric composites by dielectrophoresis. J Appl Phys. 2010;107:024107.

24. James NK, Ende D van den, Lafont U, Van der Zwaag S, Groen WA. Piezoelectric and mechanical properties of structured PZT-epoxy composites. J Mater Res. 2013;28:635-41.

25. Scholl L, Schmidig G, Faizan A, TenHuisen K, Nevelos J. Evaluation of surgical impaction technique and how it affects locking strength of the head-stem taper junction. Proc Inst Mech Eng [H]. 2016;230:661-7.

26. Kahanovitz N. The Use of Adjunctive Electrical Stimulation to Enhance the Healing of Spine Fusions. Spine. 1996;21:2523-5.

27. Martin B, Burr D, Sharkey N. Skeletal Tissue Mechanics. New York, NY: Springer-Verlag New York; 1998. 392 p.

28. SpF Spinal Fusion Stimulator | SpF Spinal Fusion Stimulator by Zimmer Biomet [Internet]. [cited 2019 Feb 25]. Available from: https://www.zimmerbiomet.com/medicalprofessionals/spine/product/spf-spinal-fusion-stimulator.html

29. Bassett CA, Pawluk RJ, Becker RO. Effects of Electric Currents on Bone in Vivo. Nature. 1964;204:652-4.

30. Dejardin LM, Kahanovitz N, Arnoczky SP, Simon BJ. The effect of varied electrical current densities on lumbar spinal fusions in dogs. Spine J. 2001;1:341-7. 
31. Hughes MS, Anglen JO. The use of implantable bone stimulators in nonunion treatment. Orthopedics. 2010;33.

32. Bodhak S, Bose S, Kinsel WC, Bandyopadhyay A. Investigation of In Vitro Bone Cell Adhesion and Proliferation on Ti Using Direct Current Stimulation. Mater Sci Eng C Mater Biol Appl. 2012;32:2163-8.

33. Fonder MA, Lazarus GS, Cowan DA, Aronson-Cook B, Kohli AR, Mamelak AJ. Treating the chronic wound: A practical approach to the care of nonhealing wounds and wound care dressings. J Am Acad Dermatol. 2008;58:185-206.

34. Thakral G, LaFontaine J, Najafi B, Talal TK, Kim P, Lavery LA. Electrical stimulation to accelerate wound healing. Diabet Foot Ankle. 2013;4.

35. Ud-Din S, Bayat A. Electrical Stimulation and Cutaneous Wound Healing: A Review of Clinical Evidence. Healthcare. 2014;2:445-67.

36. Becker RO. The bioelectric factors in amphibian-limb regeneration. J Bone Joint Surg Am. 1961;43-A:643-56.

37. Zhao M, Song B, Pu J, Wada T, Reid B, Tai G, et al. Electrical signals control wound healing through phosphatidylinositol-3-OH kinase-gamma and PTEN. Nature. 2006;442:457-60.

38. Murray PJ, Wynn TA. Protective and pathogenic functions of macrophage subsets. Nat Rev Immunol. 2011;11:723-37.

39. Hoare JI, Rajnicek AM, McCaig CD, Barker RN, Wilson HM. Electric fields are novel determinants of human macrophage functions. J Leukoc Biol. 2016;99:1141-51.

40. Lin F, Baldessari F, Gyenge CC, Sato T, Chambers RD, Santiago JG, et al. Lymphocyte electrotaxis in vitro and in vivo. J Immunol Baltim Md 1950. 2008;181:2465-71.

41. Burr HS, Taffel M, Harvey SC. An Electrometric Study of the Healing Wound in Man. Yale J Biol Med. 1940;12:483-5.

42. Li H, Tian C, Deng ZD. Energy harvesting from low frequency applications using piezoelectric materials. Appl Phys Rev. 2014;1:041301.

43. Shenck NS, Paradiso JA. Energy scavenging with shoe-mounted piezoelectrics. IEEE Micro. 2001;21:30-42.

44. APC International Ltd. Piezoelectric Ceramics Principles and Applications. 2nd ed. APC International Ltd.; 2011.114 p.

45. Anton SR, Sodano HA. A review of power harvesting using piezoelectric materials (20032006). Smart Mater Struct. 2007;16:R1-R21. 
46. Groen P, Holterman. An Introduction to Piezoelectric Materials and Components. Stichting Applied Piezo; 2012.

47. Van den Ende DA, Groen WA, Van der Zwaag S. Robust piezoelectric composites for energy harvesting in high-strain environments. J Intell Mater Syst Struct. 2013;24:2262-9.

48. Van den Ende DA, Van Kempen SE, Wu X, Groen WA, Randall CA, Van der Zwaag S. Dielectrophoretically structured piezoelectric composites with high aspect ratio piezoelectric particles inclusions. J Appl Phys. 2012;111:124107.

49. McKenna EM, Lin AS, Mickelson AR, Dinu R, Jin D. Comparison of $\mathrm{r}_{33}$ values for AJ404 films prepared with parallel plate and corona poling. JOSA B. 2007;24:2888-92.

50. Waller D, Iqbal T, Safari A. Poling of Lead Zirconate Titanate Ceramics and Flexible Piezoelectric Composites by the Corona Discharge Technique. J Am Ceram Soc. $1989 ; 72: 322-4$.

51. Banerjee S, Cook-Chennault KA, Du W, Sundar U, Halim H, Tang A. Piezoelectric and dielectric characterization of corona and contact poled PZT-epoxy-MWCNT bulk composites. Smart Mater Struct. 2016;25:115018.

52. Bhalla A, Ainger F, Pohanka R, Uchino K. Ferroelectric Materials and Their Applications to Sensors. Vol. 87. Gordon and Breach Sciece Publishers; 1988.

53. James NK, Comyn T, Hall D, Daniel L, Kleppe A, Zwaag SVD, et al. Analysis of the state of poling of lead zirconate titanate (PZT) particles in a Zn-ionomer composite.

Ferroelectrics. 2016;493:139-50.

54. Coates K. Ultrasound Stimulation of a Piezoelectric Composite with Compliant Layers on Power Output for Bone Healing in Spinal Fusion Applications [Internet] [Thesis]. University of Kansas; 2018 [cited 2019 Feb 25]. Available from:

https://kuscholarworks.ku.edu/handle/1808/27587

55. Halpin JC. Stiffness and Expansion Estimates for Oriented Short Fiber Composites. J Compos Mater. 1969;3:732-4.

56. Affdl JCH, Kardos JL. The Halpin-Tsai equations: A review. Polym Eng Sci. 1976;16:34452.

57. Yamada T, Ueda T, Kitayama T. Piezoelectricity of a high-content lead zirconate titanate/polymer composite. J Appl Phys. 1982;53:4328-32.

58. Bowen C. Dielectric properties of dielectrophoretically assembled particulate-polymer composites. JMR. 2011;13:205-10. 
59. Krech ED, Barrett RM, Cadel ES, Friis EA. Power Generation Amplification and Stack Toughening via Compliant Layer Interdigitation. In American Society of Mechanical Engineers; 2018.

60. Heiney JP, Battula S, Vrabec GA, Parikh A, Blice R, Schoenfeld AJ, et al. Impact magnitudes applied by surgeons and their importance when applying the femoral head onto the Morse taper for total hip arthroplasty. Arch Orthop Trauma Surg. 2009;129:793-6.

61. Hernández-Vaquero D, Suárez-Vázquez A, Iglesias-Fernández S, García-García J, CerveroSuárez J. Dynamisation and early weight-bearing in tibial reamed intramedullary nailing: Its safety and effect on fracture union. Injury. 2012;43:S63-7.

62. Kempf I, Grosse A, Beck G. Closed locked intramedullary nailing. Its application to comminuted fractures of the femur. J Bone Joint Surg Am. 1985;67:709-20.

63. Arazi M, Oğün TC, Oktar MN, Memik R, Kutlu A. Early weight-bearing after statically locked reamed intramedullary nailing of comminuted femoral fractures: is it a safe procedure? J Trauma. 2001;50:711-6.

64. Denisov A, Yeatman E. Ultrasonic vs. Inductive Power Delivery for Miniature Biomedical Implants. In: 2010 International Conference on Body Sensor Networks. 2010. p. 84-9. 


\section{Appendix A: MATLAB Code}

\section{A.1: Volume Fraction Code}

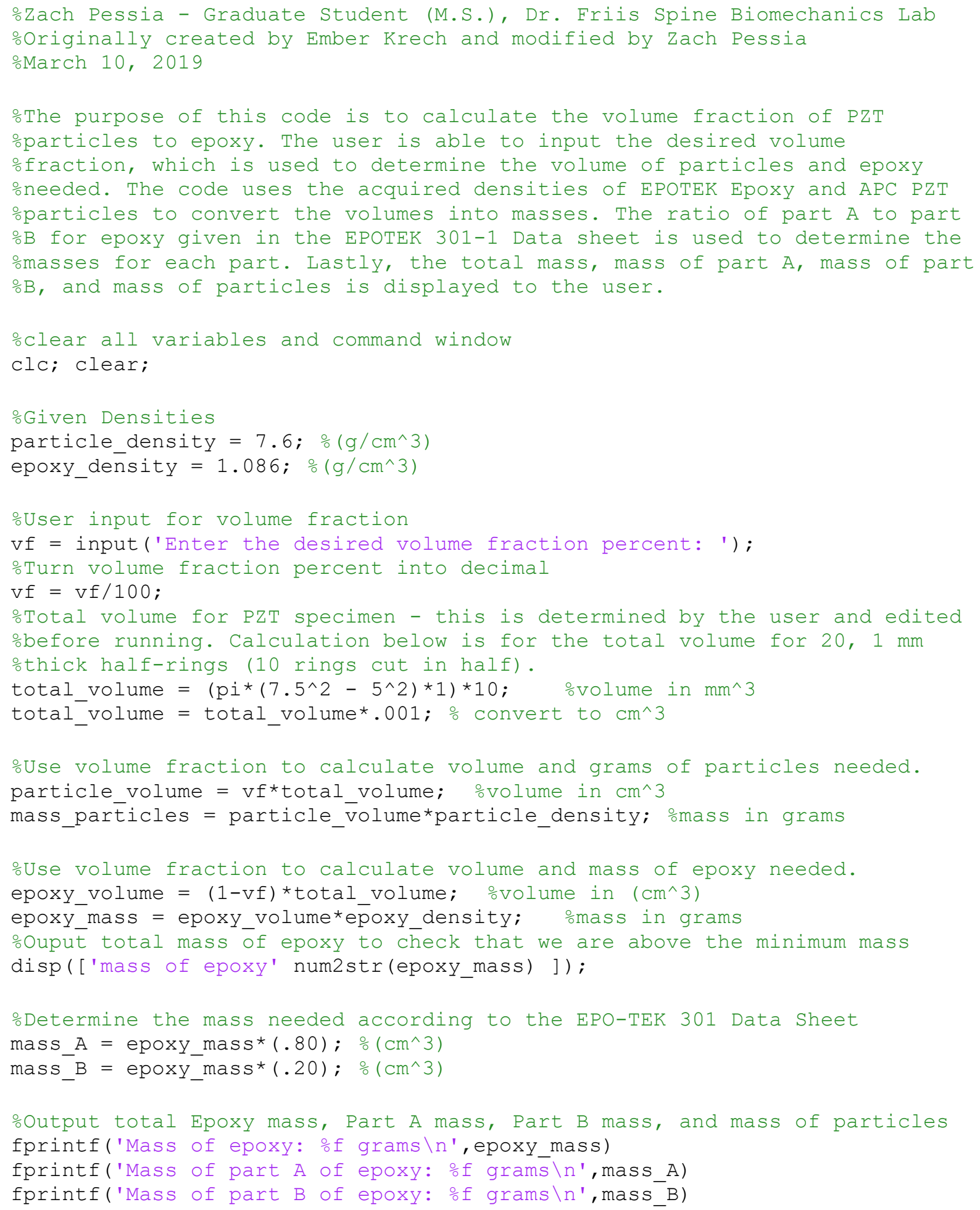


fprintf('Mass of particles: f grams $\backslash n$ ', mass_particles)

\section{A.2: Power Analysis Code}

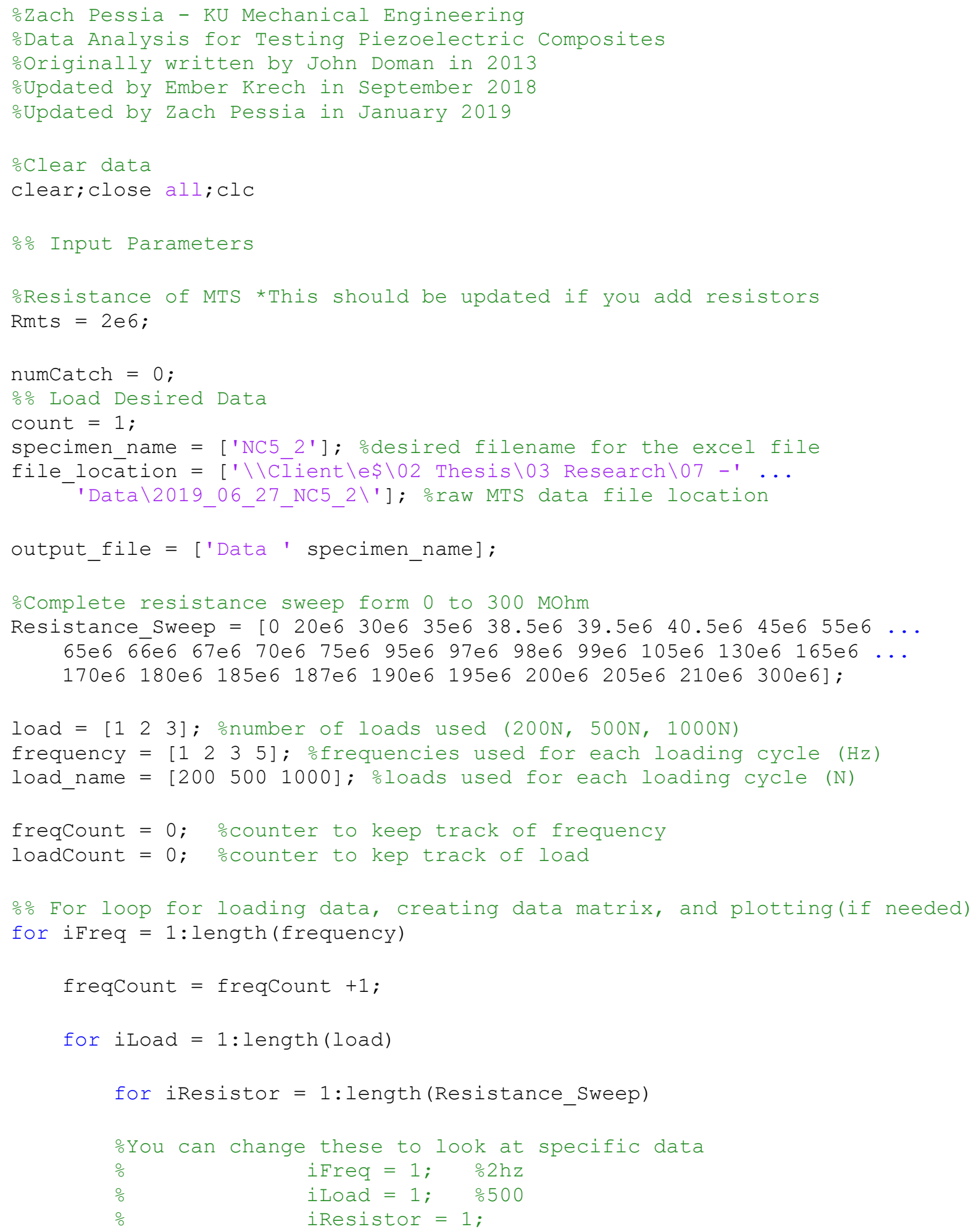




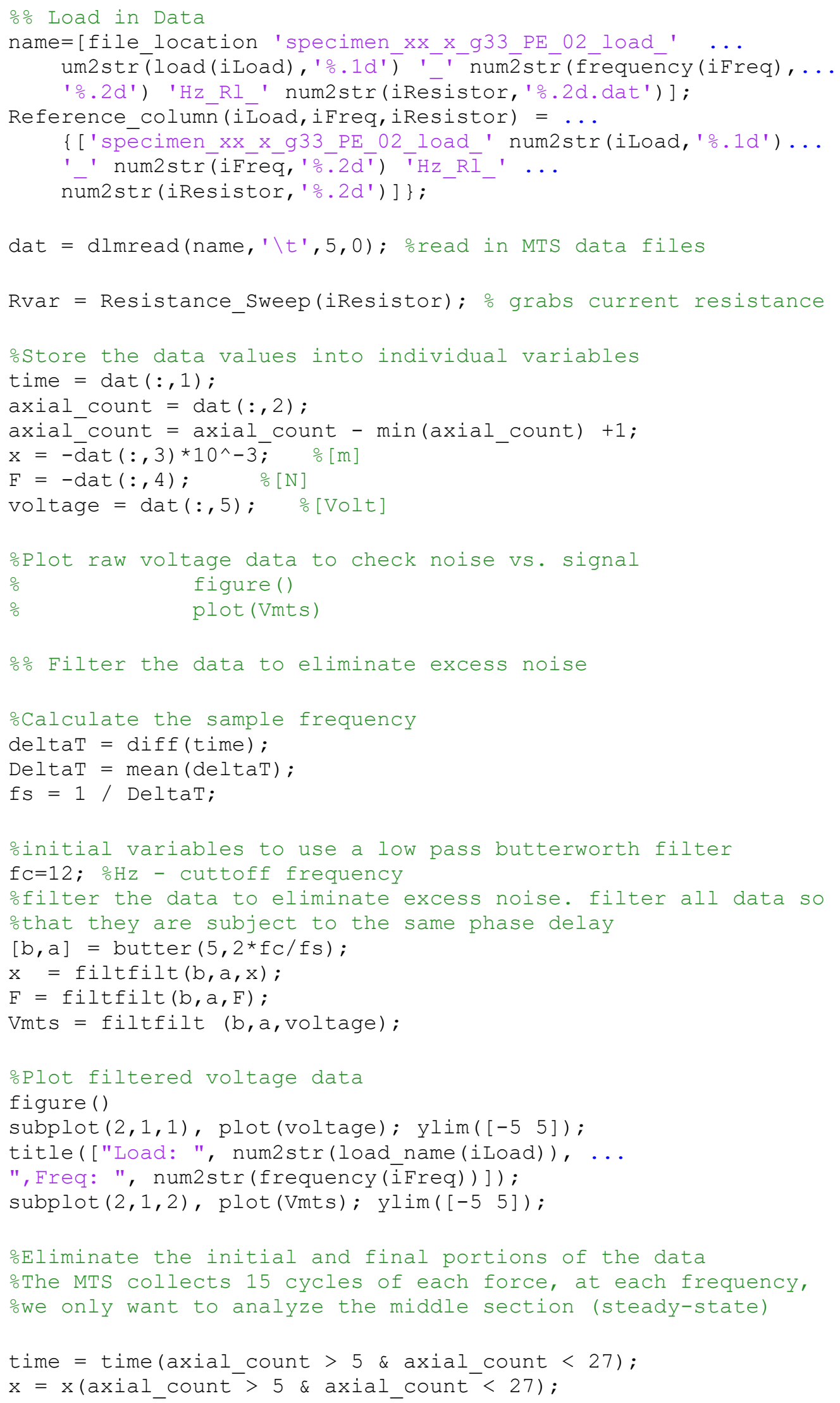




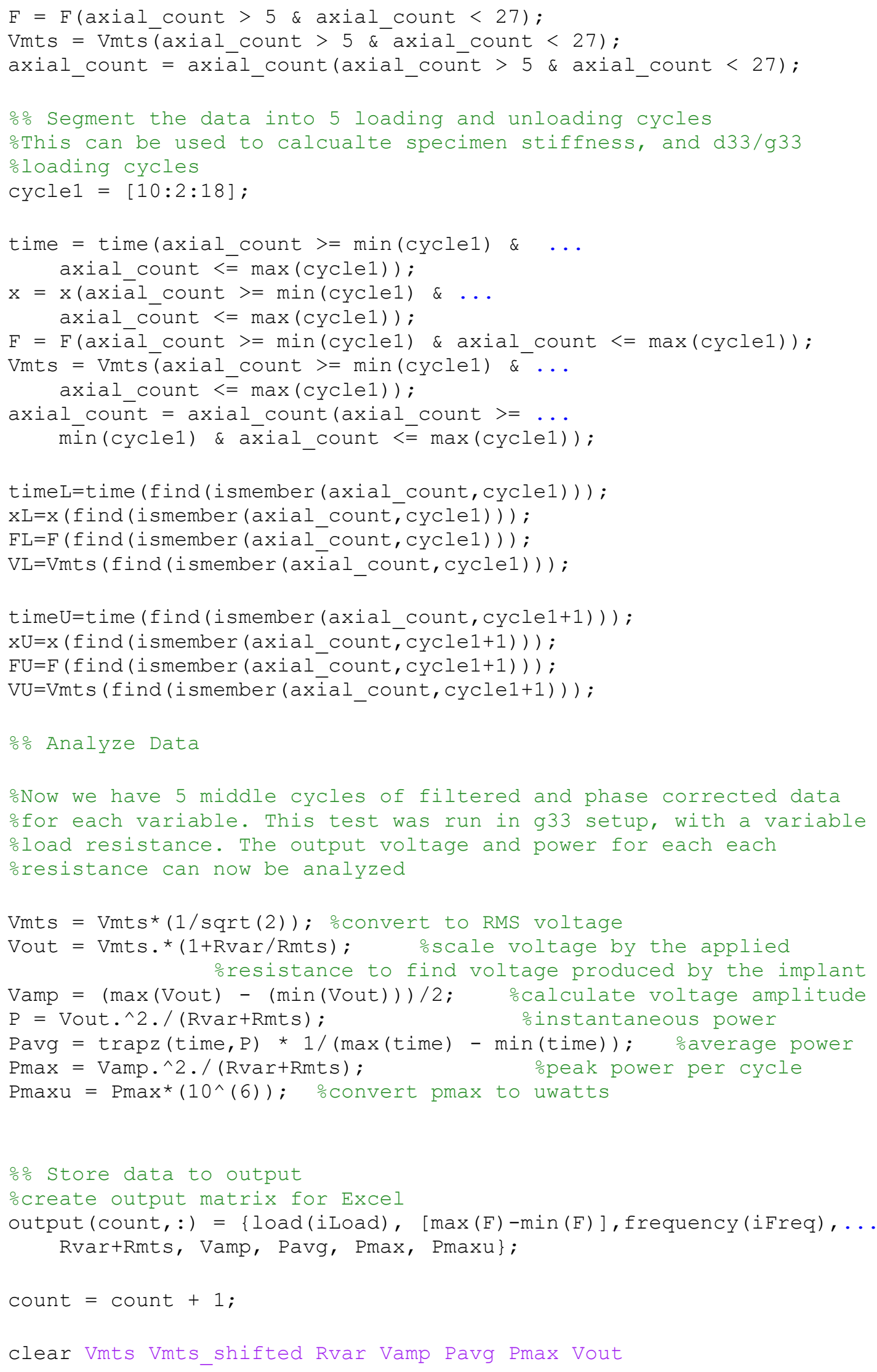


end

end

end

$\therefore$ Output to an excel sheet for later analysis

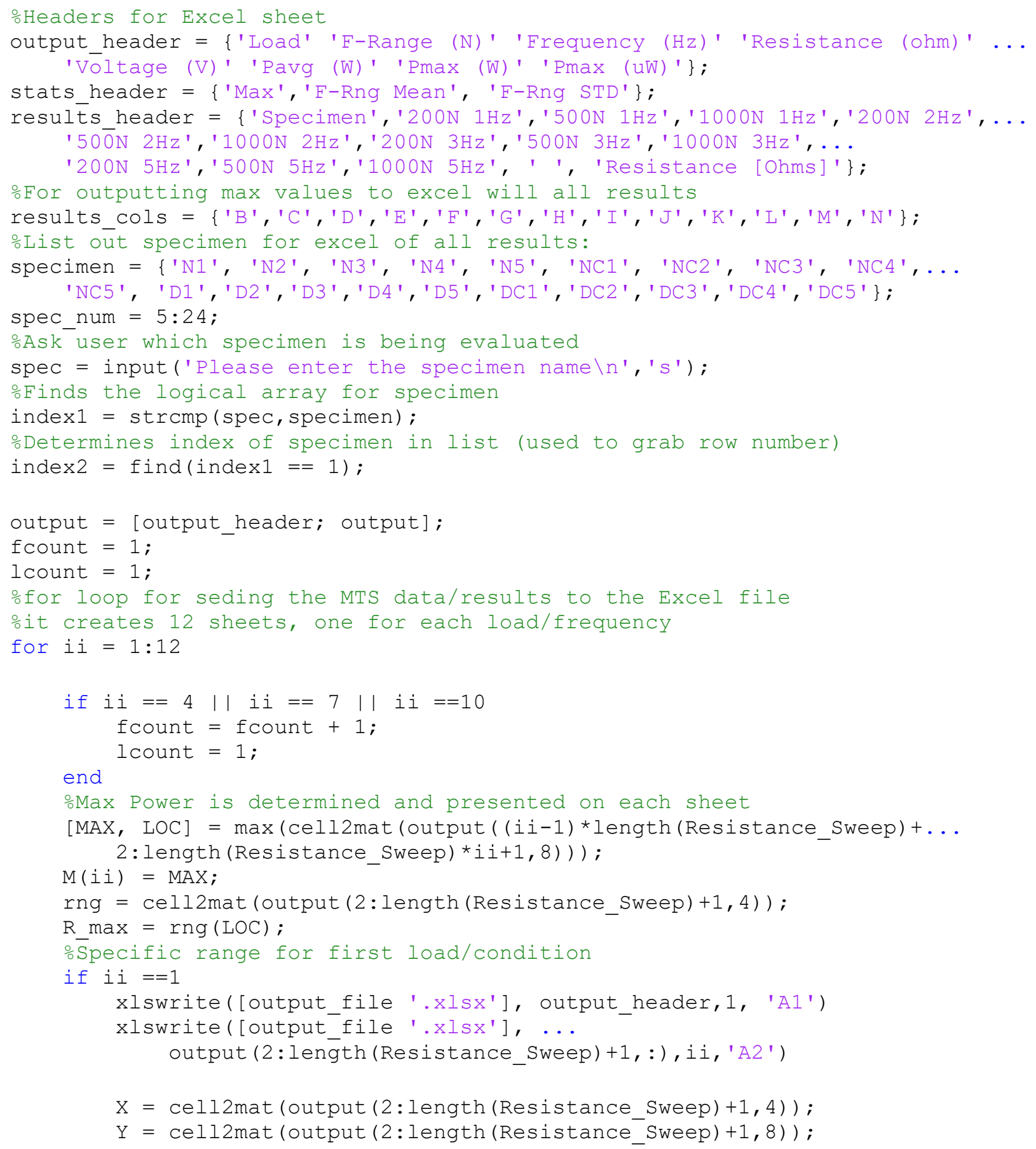




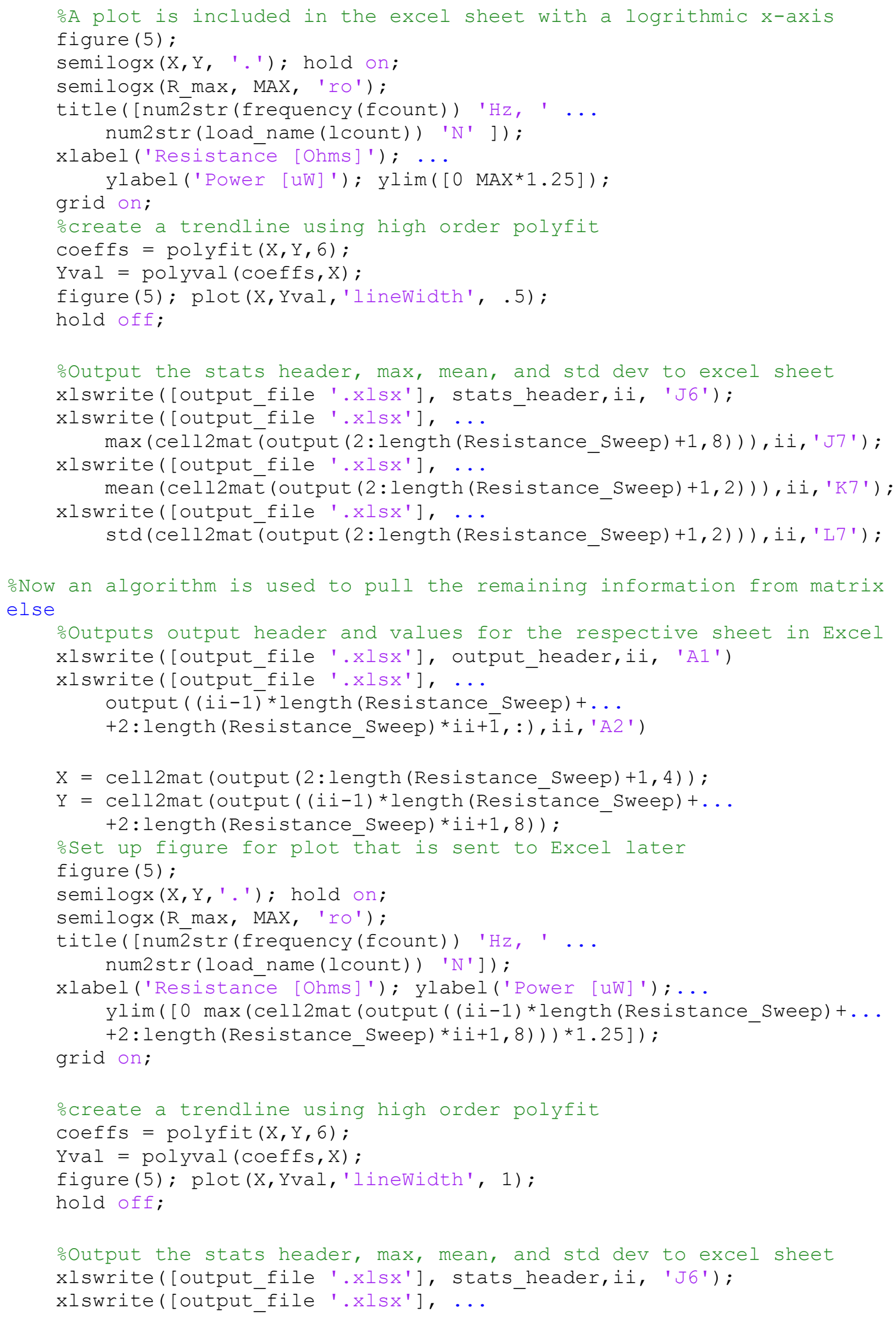




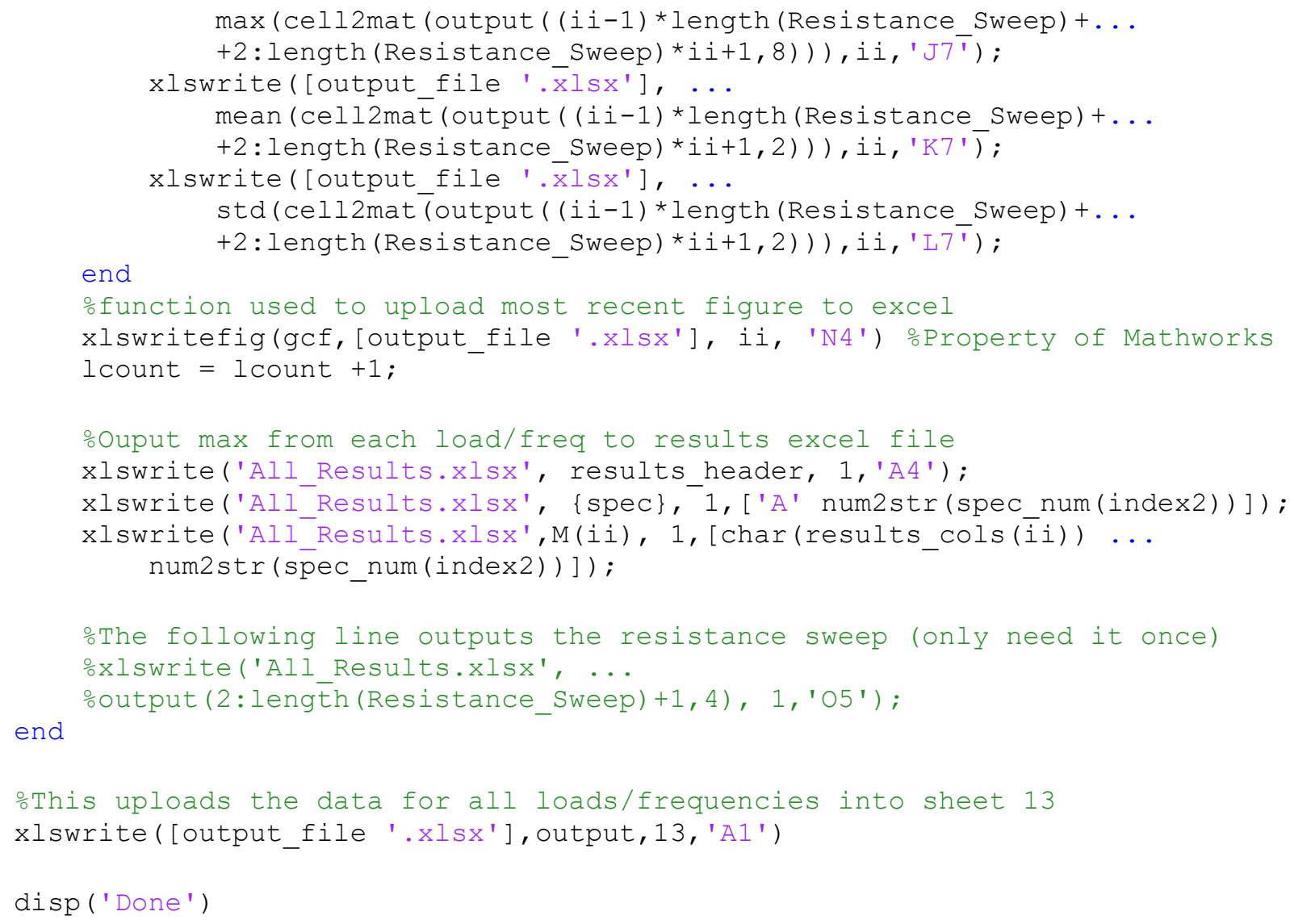

\section{A.3: Summary File Code}

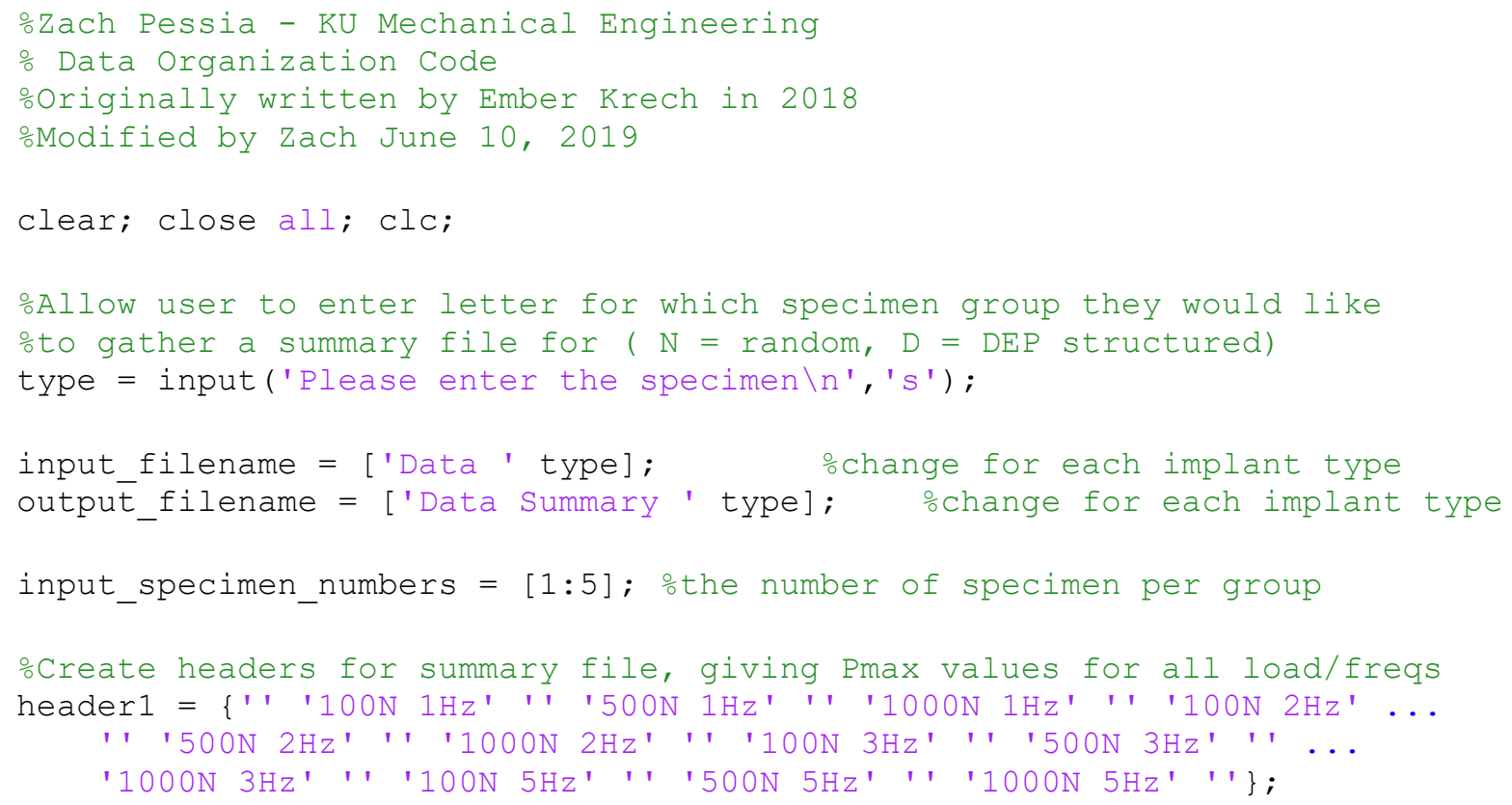




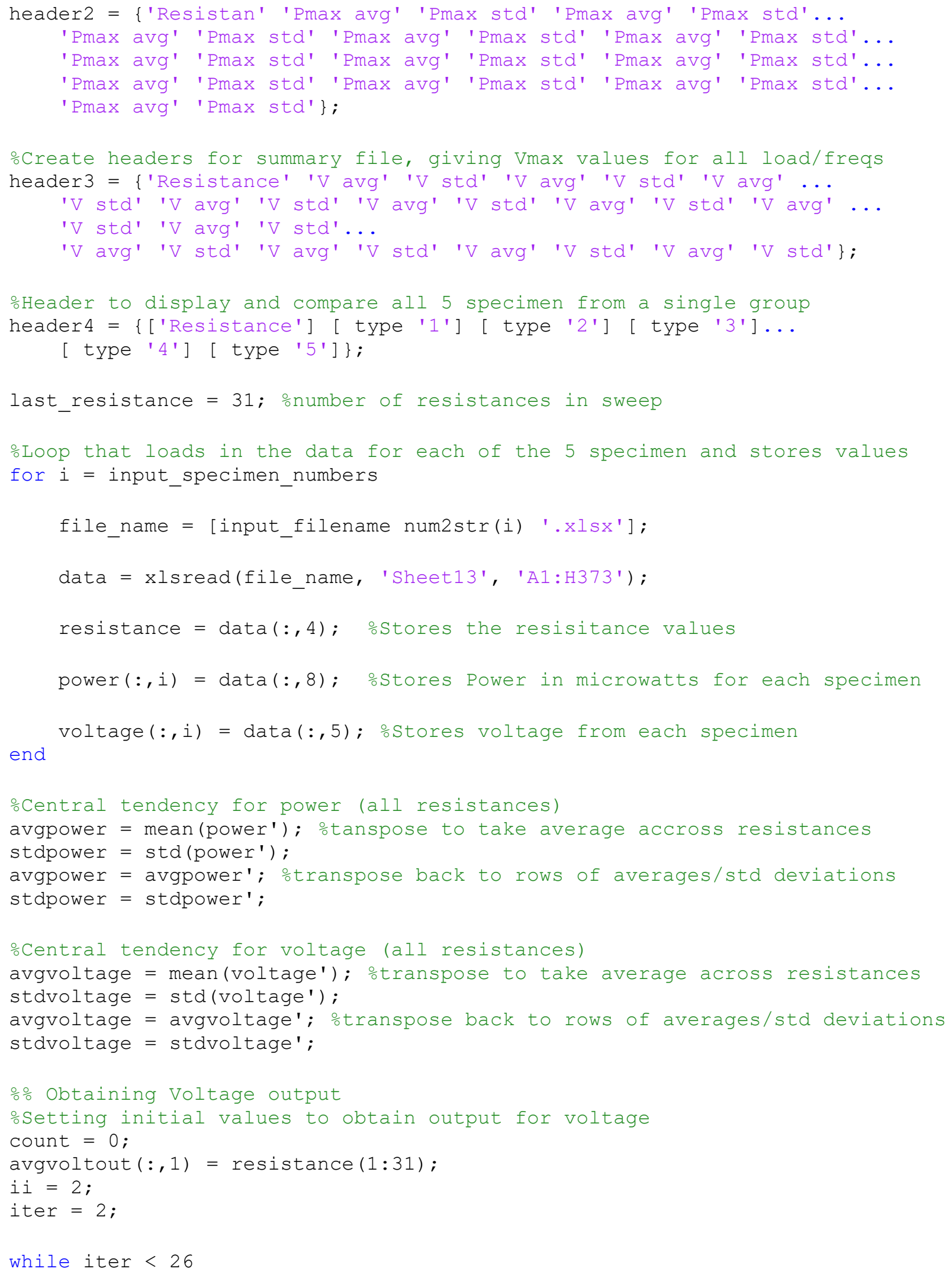




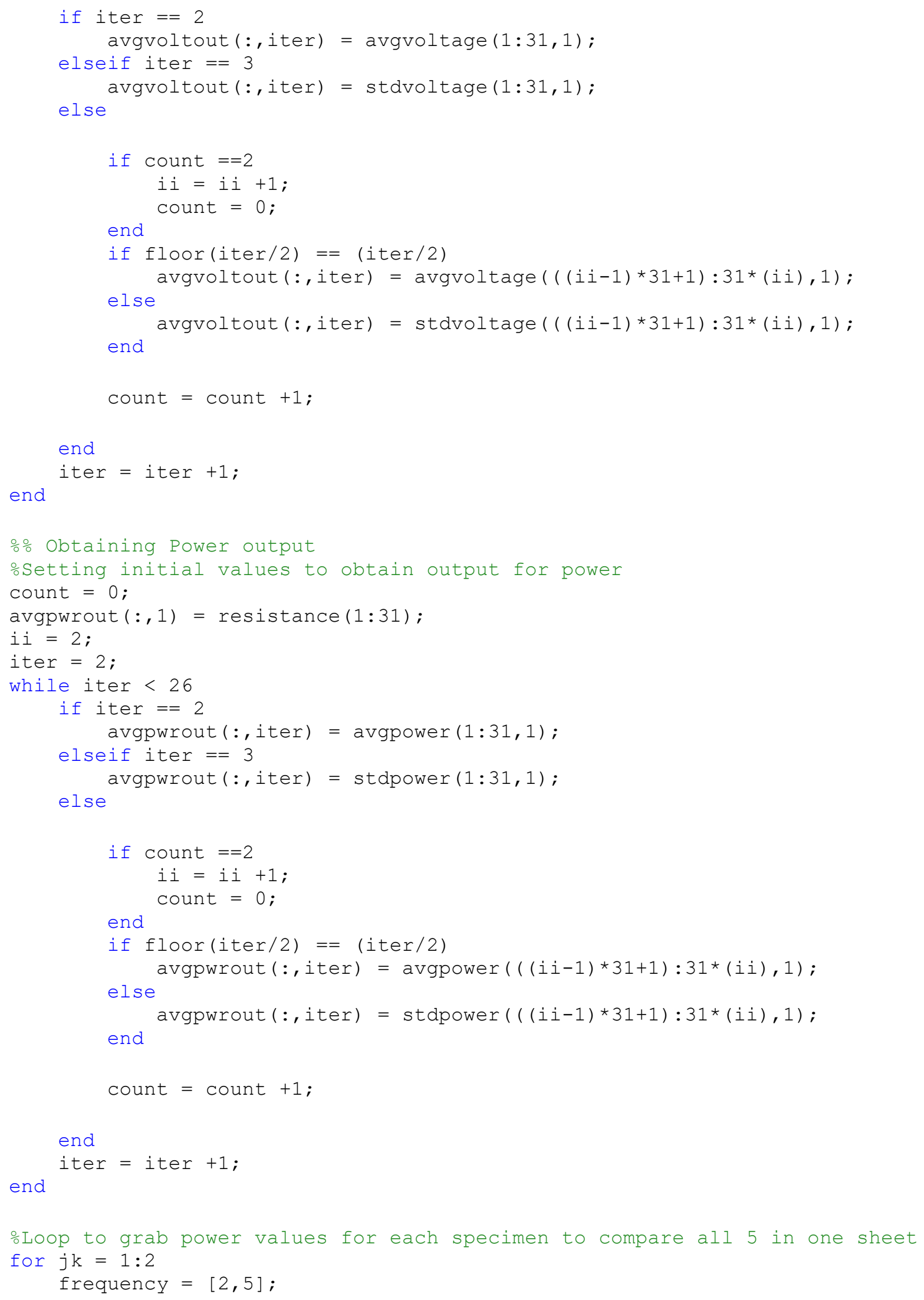




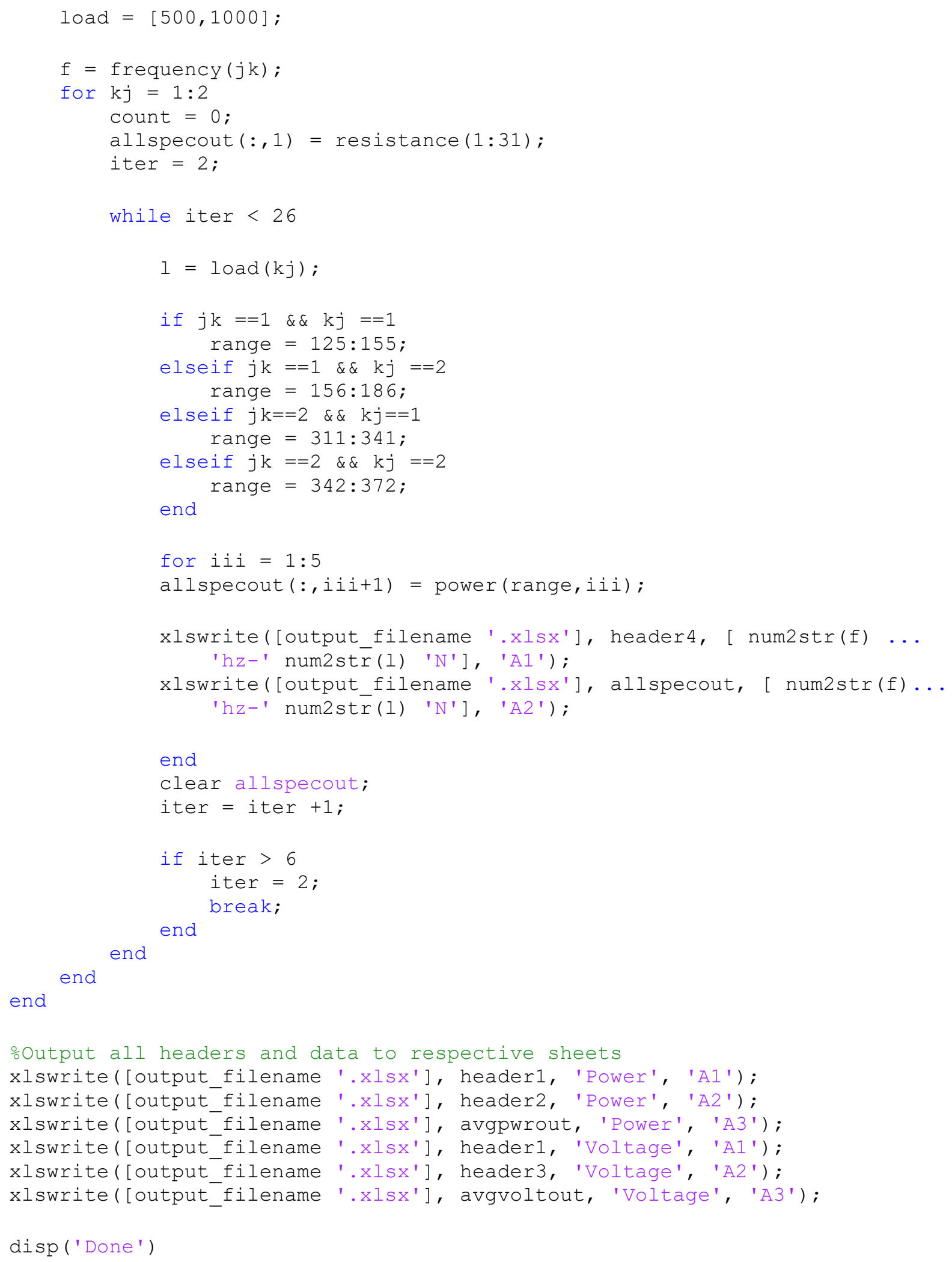




\section{A.4: Statistics Preparation Code}

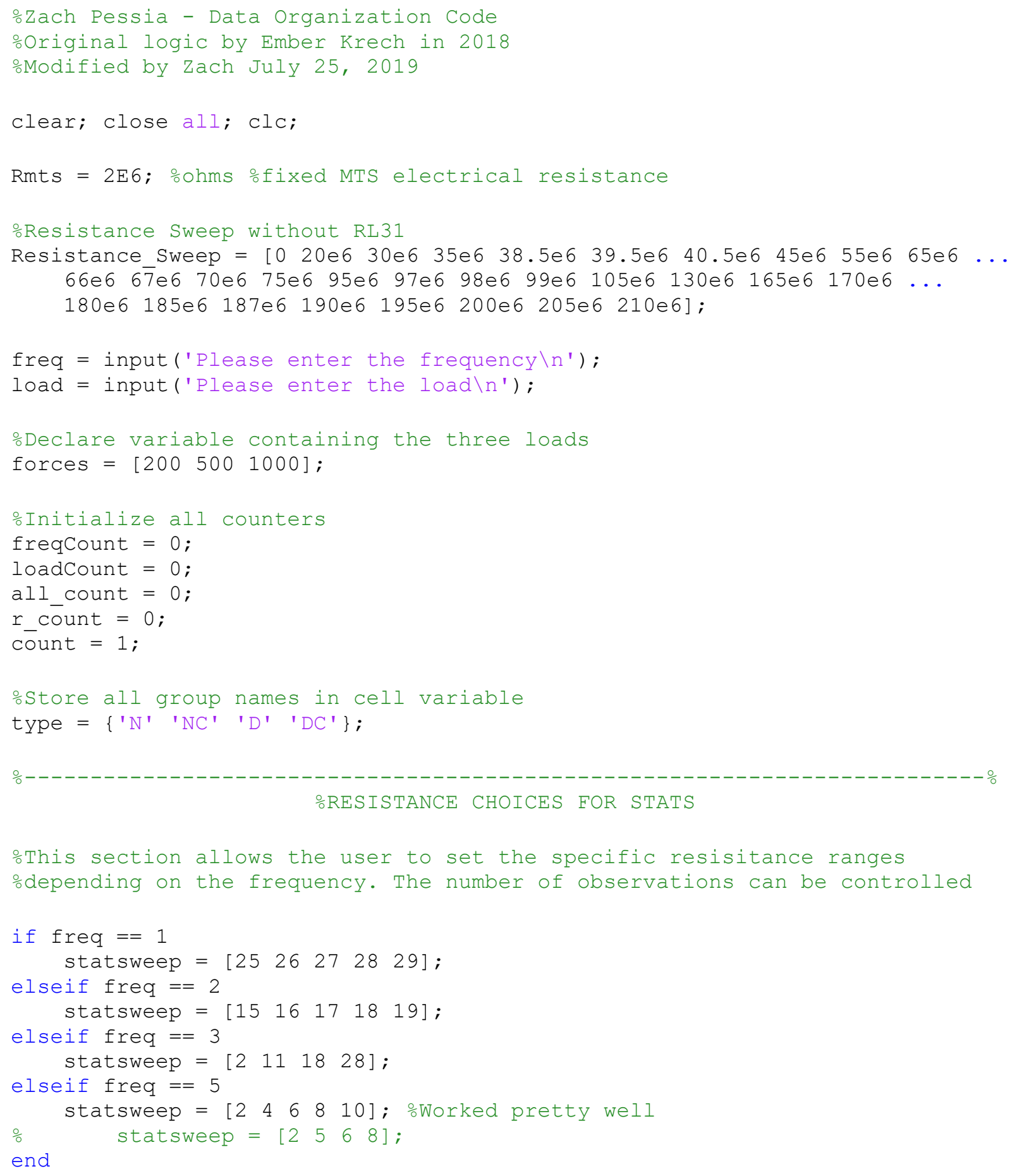




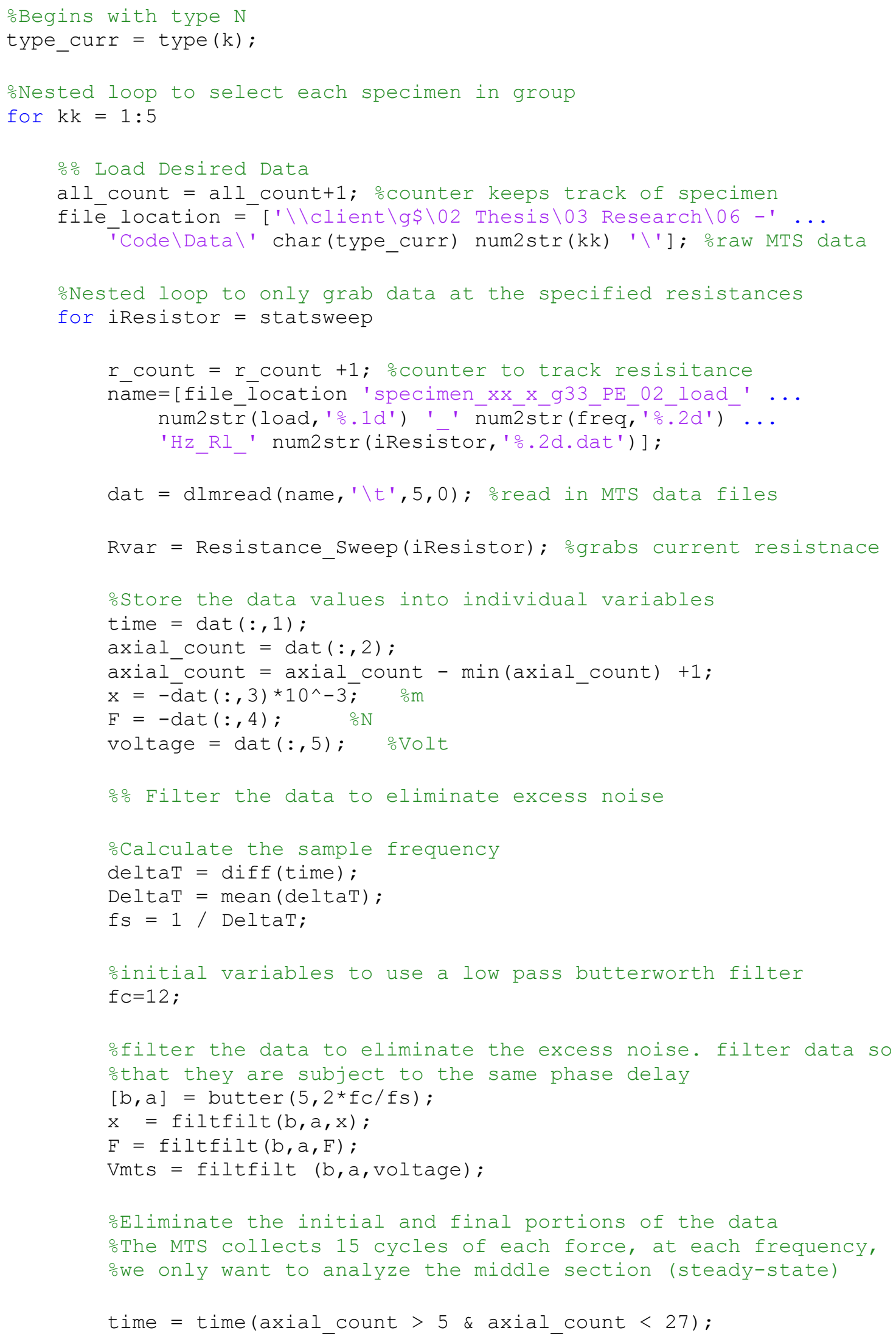




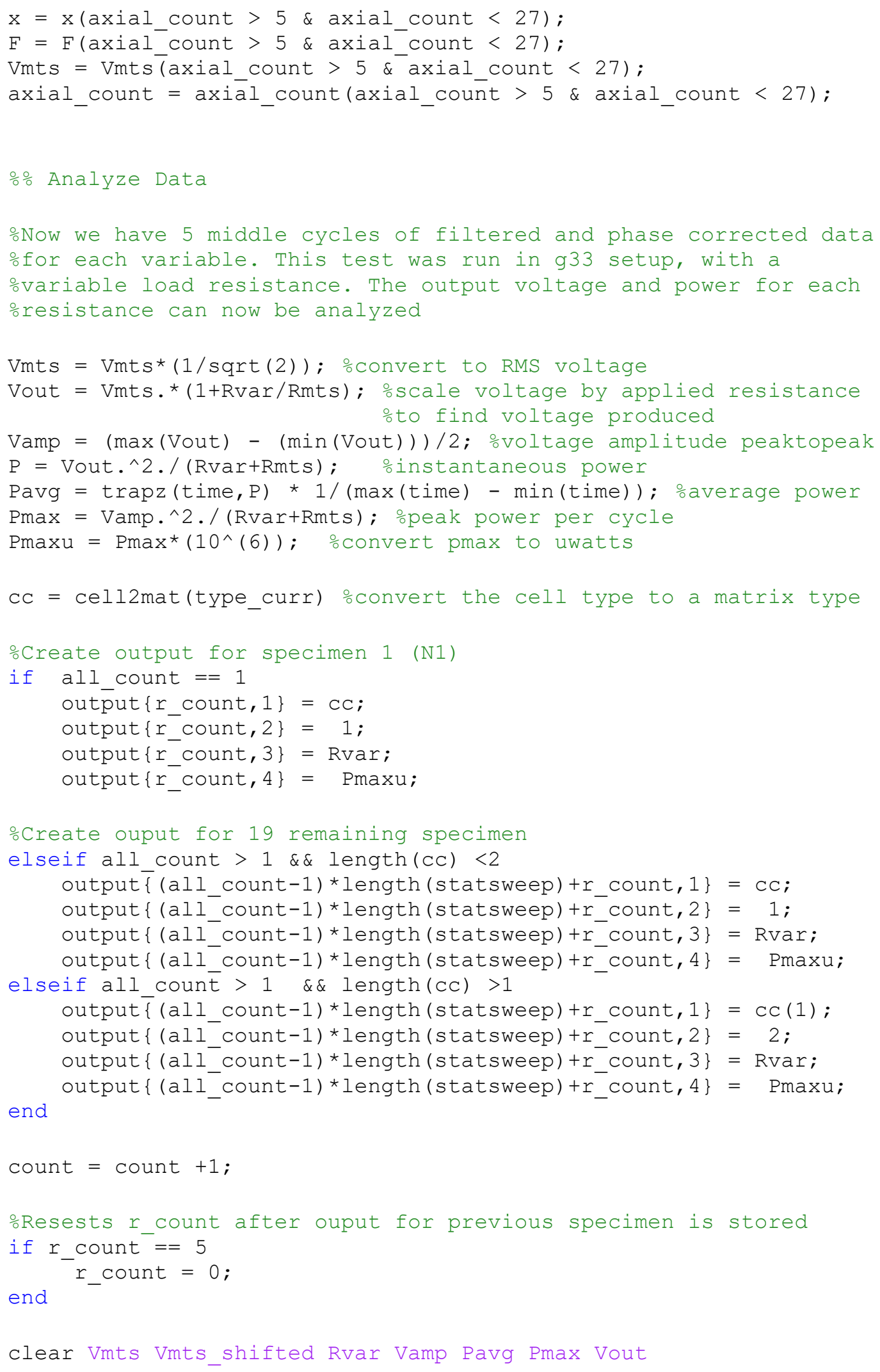




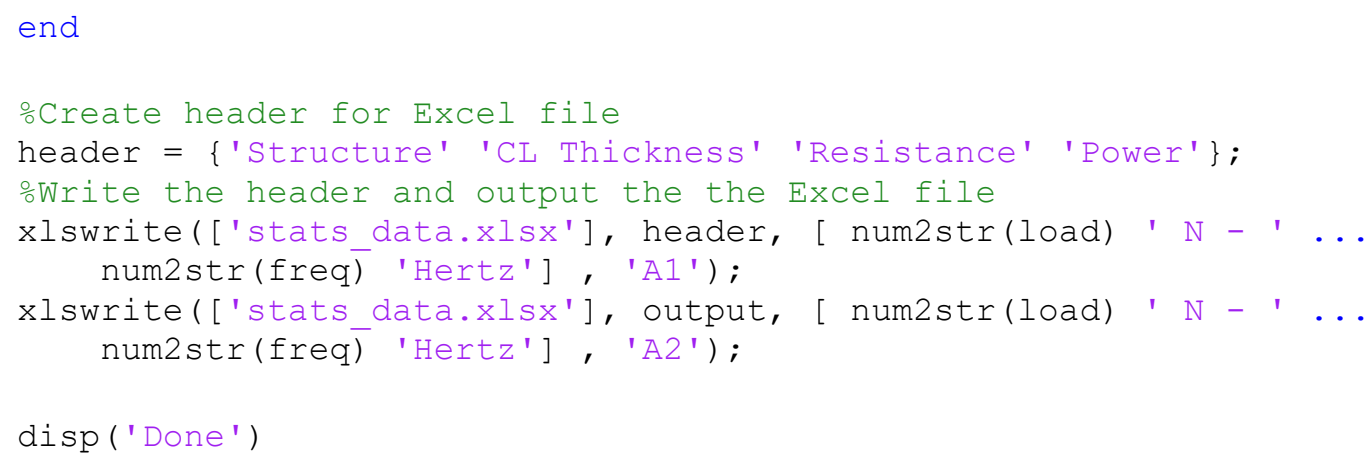

\section{Appendix B: SAS Code}

The following codes were implemented to investigate statistical differences between experimental results. The SAS power code was initially implemented, and impossibly large sample sizes would be required to achieve statistical power to detect differences between groups. This is due to the relatively low power production and high standard deviations observed. When applicable, the Wilcoxon rank-sum test was used and the statistical preparation MATLAB code (A.4) was used to prepare data files for SAS.

\section{B.1 Power Analysis Code}

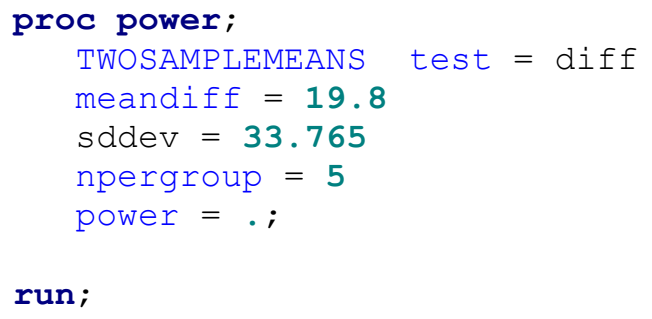

\section{B.2 Wilcoxon Rank-Sum Code}

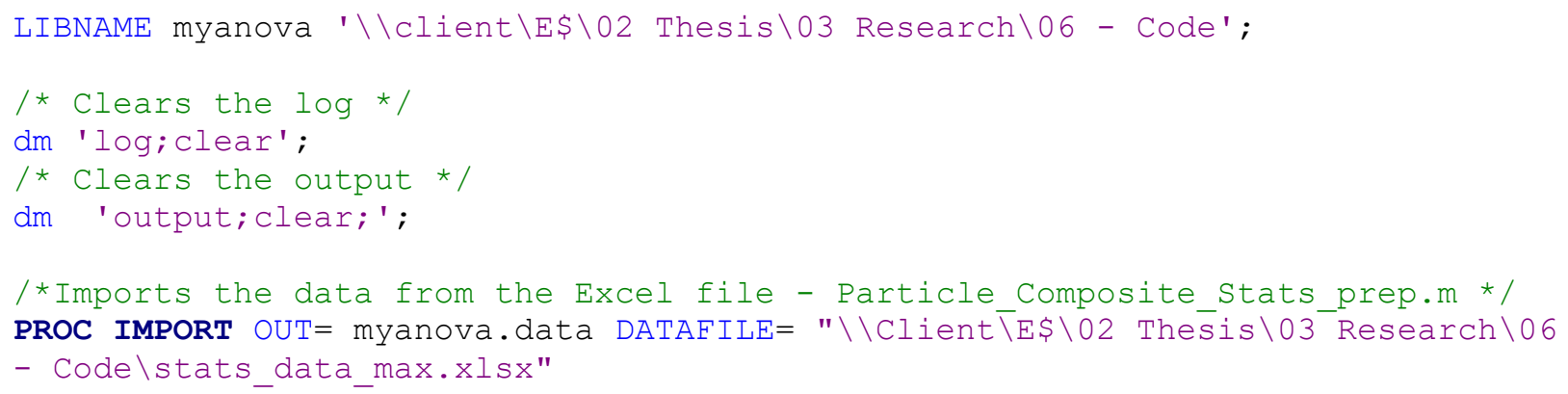




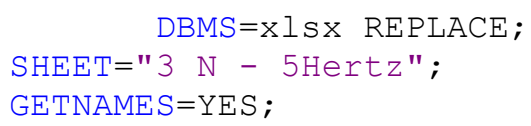

RUN； 


\section{Appendix C: Ring Particle Composites}

This section of the Appendix discusses the initial development of PZT particle composites for implementation into an intramedullary nail and is suggested for future work. This application of PZT particles was investigated for multiple months, but was eventually abandoned in order to develop and characterize the power generation of circular composite discs structured by dielectrophoresis (DEP) in compliant layer adaptive composite stacks (CLACS). The direction of the study also shifted in order to directly compare power generated to the original CLACS study by Krech et al.

\section{C.1: Fracture Fixation Implementation}

Before manufacturing PZT composite CLACS, the novel design of an Intramedullary (IM) nail was investigated to determine how the PZT composite CLACS would be implemented into fracture fixation devices. The overall goal was to design a modular intramedullary nail with a PZT composite exchangeable insert.

The first implementation of this design was a black box idea that comprised a traditional IM nail with a PZT insert located in the middle (Figure A.1). With this design, the nail is modular and allows different lengths of top or bottom rod. This allows the PZT insert to be moved along the length of the rod to provide DC stimulation directly at the fracture site. The specific design of how the PZT insert is incorporated into the nail and how it satisfies the loading conditions will be discussed in the upcoming study by Cunningham et al.. Due to the unique application, the piezoelectric insert was

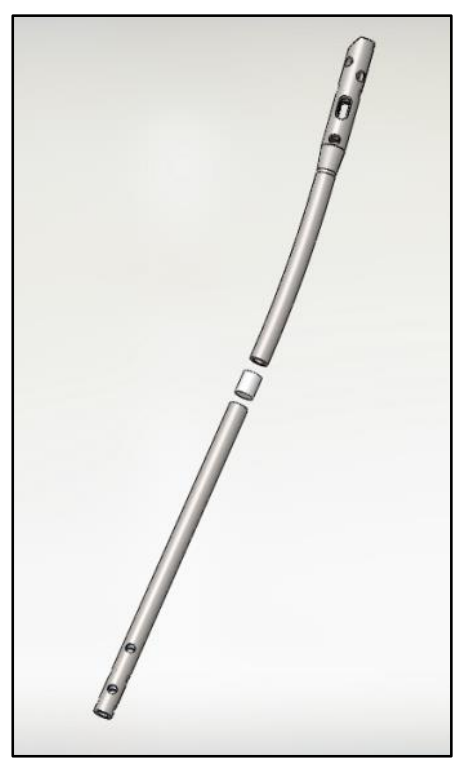

Figure A.1: Original modular IM nail. 
determined to be ring shaped in order to allow a guide wire to pass through during surgical implantation.

\section{C.2: Half Ring Development}

Piezoelectric composites are desirable for the IM nail due to the high impact loads from implantation $^{60}$. In addition, the possible presence of torsional or bending loads on the PZT would be troublesome for the commonly used, brittle, PZT ceramics. Piezoelectric particle composite rings of $16 \mathrm{~mm}$ OD, $5 \mathrm{~mm} \mathrm{ID}$, and $1 \mathrm{~mm}$ thickness were manufactured at varying volume fractions. However; the impedance was very high due to the lack of connectivity in the composite and high thickness. This led to the development of half ring piezoelectric composites.

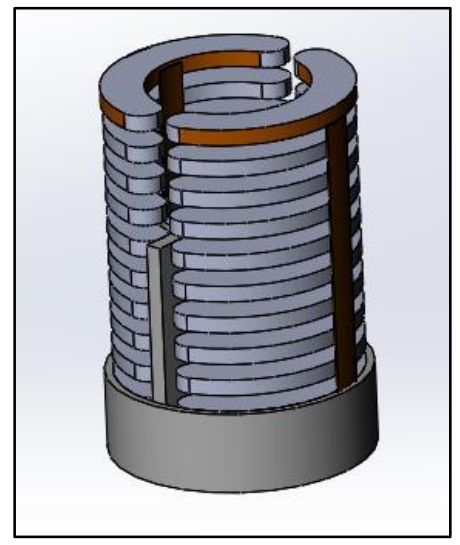

Figure A.2: Half ring stacking concept with epoxy support shown in Figure 19.

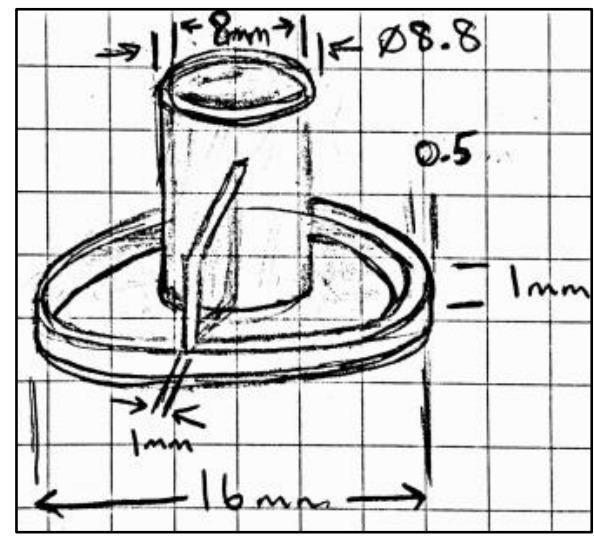

Figure A.3 Epoxy encapsulation support concept for half rings or rings

Using half rings doubles the amount of parallel connection, thereby reducing the impedance significantly. Figure A.3 shows the drawing for an epoxy encapsulation support. This novel tool helps to center half rings (or whole rings) while also integrating a constant bottom encapsulation. This tool also guarantees the stack will cure centered and level. The other reason for investigating is the ability to radially pole the rings. Recent studies have observed radially poled discs in CLACS producing more power compared to thickness poled discs at low frequencies. 
The procedure for manufacturing particle composite half rings (or any custom shape composite) is described in Appendix D.

If rings or half rings are to be investigated in the future, it is suggested to use very small thicknesses $(0.4 \mathrm{~mm}-0.6 \mathrm{~mm})$ due to poling efficiency and improved effect of DEP across lower thicknesses. Radially poling vs. thickness poling for such composites could also be investigated with the half ring designs. Nevertheless, the ability to significantly reduce the impedance is desirable for circuit integration. 


\section{Appendix D: Procedures}

The following section describes all of the processes for manufacturing PZT particle composites, performing DEP, and poling. These procedures are the result of over a year of developing methods to produce successful composite materials.

\section{D.1: Making the Teflon mold}

HAZARD: When working with piezoelectric materials, proper Personal Protective Equipment (PPE) must be used. Gloves must be worn at all times when working to protect the specimen and to protect researchers from contact with lead. Proper NIOSH-approved respirators must be worn when handling PZT powder, sanding PZT composites, and using conductive spray.

1. Acquire a sheet of Teflon that is the desired thickness of the composite layer.

a. In previous studies, a $0.4 \mathrm{~mm}$ thick piece of Teflon produced $0.7 \mathrm{~mm}$ thick discs.

Therefore A thickness of Teflon slightly thinner than goal thickness is suggested to reduce post processing time.

2. Using a hole punch of the desired diameter or a custom shaped punch tool, punch the cutouts in two rows (Figure A.4).

a. The mold should fit as many discs as possible, but must fit between two delrin plates

b. There must be at least a $2 \mathrm{~mm}$ gap between discs and a $5 \mathrm{~mm}$ gap on ends to avoid shorting electrodes.

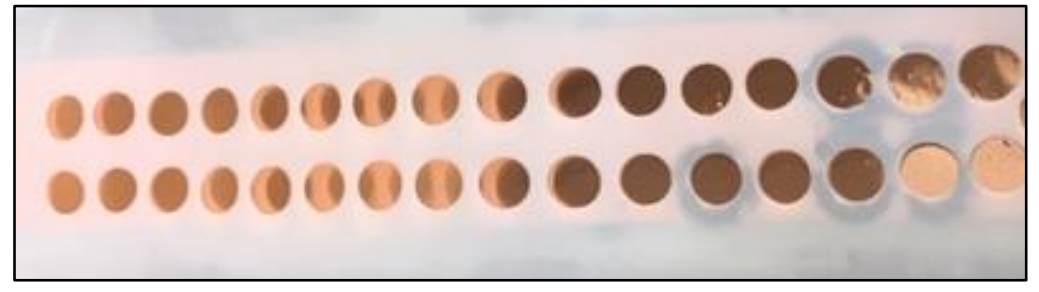

Figure A.4: Teflon mold for curing composites 


\section{D.2: Preparing the particle composite}

1. Use the volume fraction MATLAB code in Appendix A.1 to obtain the required masses of Part A epoxy, Part B epoxy, and particles for any desired volume fraction.

a. Check the calculation for volume (default: $10 \mathrm{~mm}$ diameter, $0.4 \mathrm{~mm}$ thick discs)

b. Update the density of particles and epoxy (default: APC 850, EPO-TEK 301)

2. Preheat the pot furnace to $700{ }^{\circ} \mathrm{C}$ for 30 minutes, or until temperature is held

3. Use the scale to measure required weight of particles and placed in a medium crucible.

a. It is best to overshoot mass of particles since some mass will be burned in sintering process.

4. Place crucible in pot furnace, using thermal gloves and tongs, for 1 hour to burn off organic binder in powder.

a. The powder should change to a yellow color when sintering

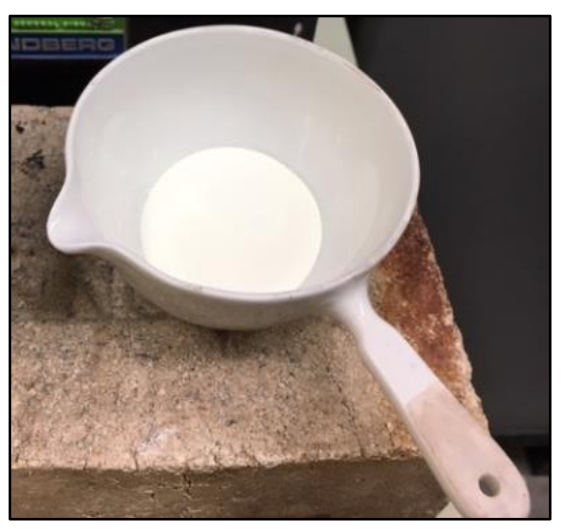

Figure A.5: Crucible with particle powder for sintering

5. While the particles are in the oven, prep the delrin plates, Teflon mold, and electrodes for filling and DEP structuring.

a. Cut one copper electrode to the necessary thickness and length. 
b. Cut one aluminum foil electrode to the necessary thickness and length.

c. Clean the top surface of each with alcohol.

d. Tape the copper electrode on one end to a flat piece of delrin.

e. Clean the Teflon mold with alcohol and place on the copper foil. Ensure one end of the copper is completely covered by Teflon to avoid shorting with aluminum electrode after molds are filled.

f. The edges of the Teflon mold may also be taped to delrin to flatten and secure it.

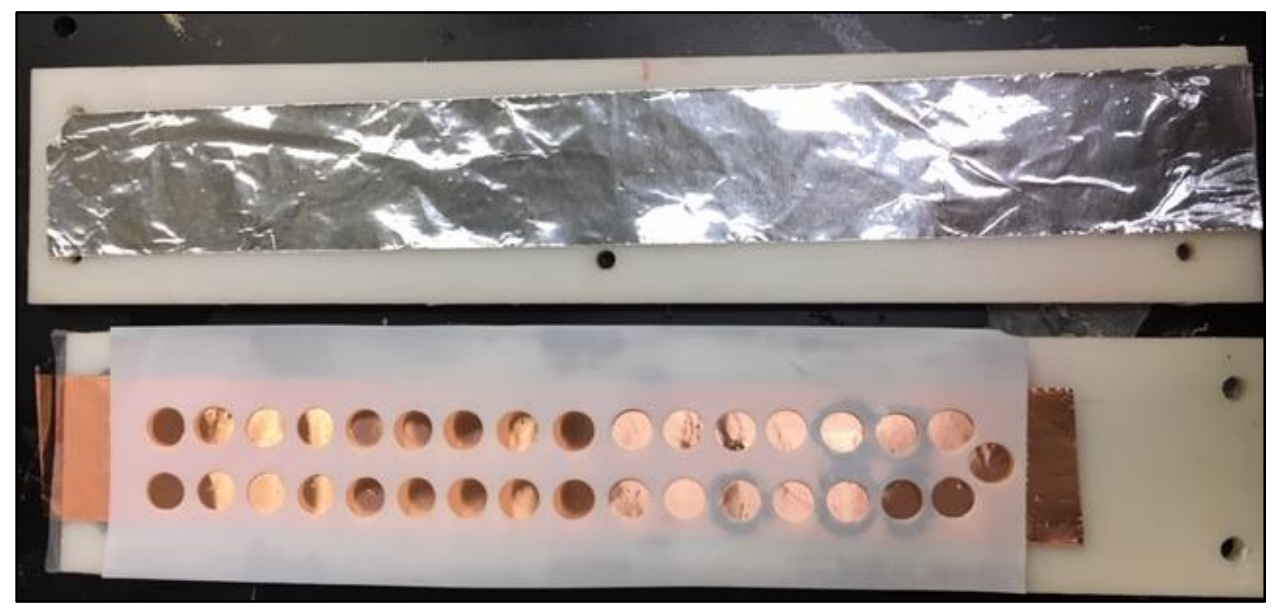

Figure A.6: Delrin plates with electrodes and Teflon mold used for curing composites and performing DEP.

6. Remove crucible and place on firebrick to cool for 15 minutes or until radiant heat dissipates and it can be touched.

a. The particles should return to a pure white color

7. Use a mortar and pestle to grind particle powder for 5 minutes.

a. Use light pressure and grind until agglomerates are broken down and individual particles or fine powder is achieved.

b. This must be done wet, or with alcohol/similar evaporating liquid 
8. Make epoxy.

a. Mix Part A and Part B (minimum batch is 3 to 5 grams).

b. Mix in a plastic cup with a metal spatula. Avoid touching edges or bottom of cup, stir slowly, and mix until two components have fully combined to a clear epoxy.

9. Pour exact mass of epoxy needed into a sturdy vessel.

a. A small crucible cup was used in previous study.

b. Total mass of mixed epoxy needed $=$ mass of $A+$ mass of $B$.

10. Mix in particles.

a. Scoop and stir very slowly with a metal spatula for 10-15 minutes or until the slurry changes to a pure white color and becomes stiff like a putty or paste.

11. Once mixed, use the spatula to scoop the slurry/putty into the Teflon mold.

a. Fill one cut-out at a time. Over apply slightly, apply some compression, and scrape off excess to leave a smooth surface.

12. Place the aluminum electrode over the mold, ensuring the top edge of the aluminum electrode does not lie past the silicone to avoid shorting with the copper.

13. Place the top piece of delrin over the aluminum electrode and place the setup on the hotplate.

14. Set the hotplate to $65^{\circ} \mathrm{C}$

15. Apply 20-35 lbs of weight stacked evenly on the top delrin piece.

16. For $0-3$, or random composites, turn hotplate off after 3 hours and let mold cure for total of 24 hours. For DEP structured composites, begin the DEP procedure. 


\section{D.3: Dielectrophoresis Procedure}

1. Check the resistance across the electrodes with a voltmeter to ensure the electrodes are not shorting, and then connect the positive and negative clips for the high voltage amplifier.

2. Turn on the function generator and Oscilloscope.

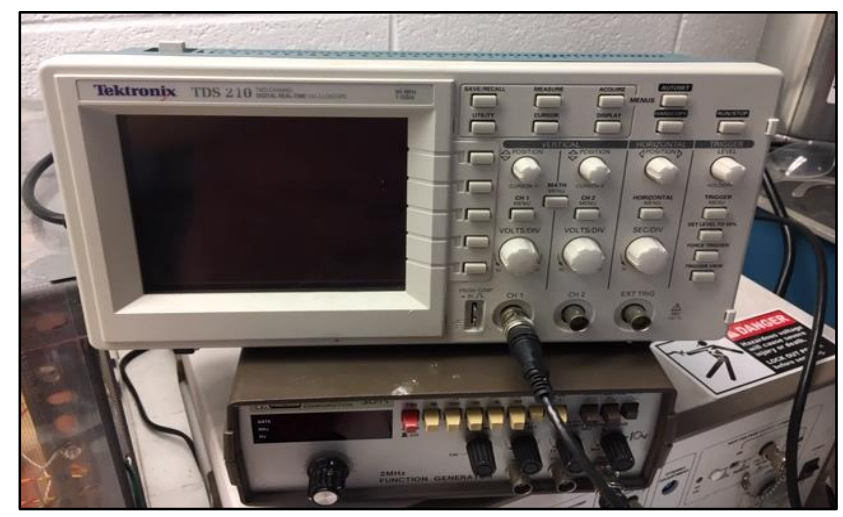

Figure A.7: Oscilloscope (top) and function generator (bottom).

3. First, connect the output of the function generator to channel 1 of the oscilloscope to check the sine wave.

a. Set the frequency of the function generator to $2000 \mathrm{~Hz}$ and the voltage to 800

Volts $(2 \mathrm{kV} / \mathrm{mm})$. Verify these numbers with the readings from the oscilloscope.

4. Connect the output of the function generator to the input of the HV amplifier and connect the voltage reading output of the HV amplifier to the oscilloscope.

5. Check that the ground has a secure connection and is properly connected all the way out the window to the post in the ground. Also check that the HV positive and negative cables are not both in contact with metal. 


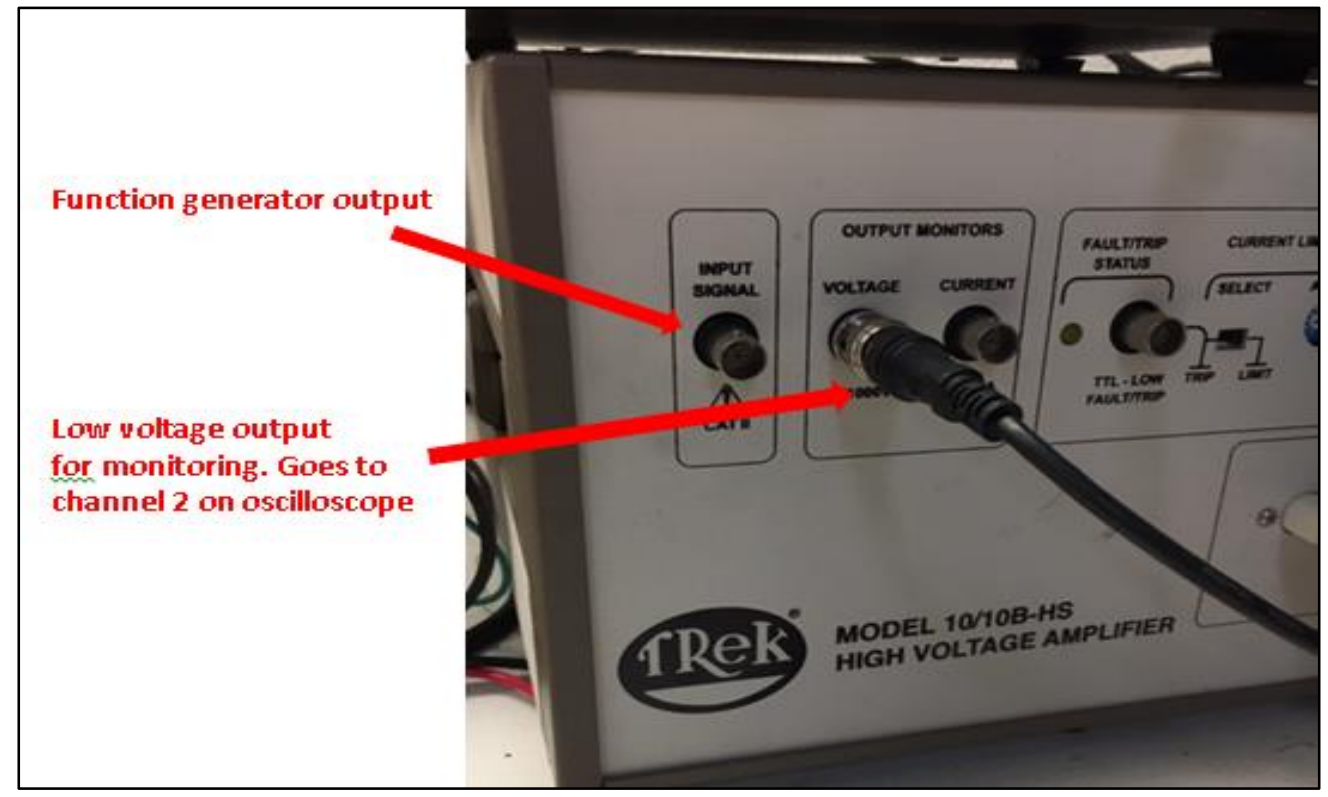

Figure A.8: HV amplifier input form function generator and output for monitoring sine wave.

6. With silicone gloves on, put on the HV protection rubber glove on your right hand, and flip the HV amplifier switch. Then, pull out the big gray stop button.

7. When everything is on and ready, press the small gray "HV ON" button.

8. Watch the Oscilloscope output.

a. There should be a sine curve. If there is no sine curve, the system is shorting.

9. Perform DEP for 3 hours at $65^{\circ} \mathrm{C}$

10. Then, turn off the hotplate with the HV glove on, and perform DEP for 1 hour as the setup cools to room temperature.

11. With your right hand double-gloved, press the big gray stop button. Then flip the power switch on the amplifier off. Once the amplifier is off, the HV glove can be removed, and the function generator and oscilloscope can be turned off.

12. Removed positive and negative leads and let sit for 20 hours. 


\section{D.4: Electrode Placement}

1. Sand composite discs to desired thickness

2. Clean with alcohol

3. Place anywhere from 10 to 30 discs on Teflon spray mat.

a. Ensure discs are as close as possible, limiting gaps.

4. Place in fume hood and turn on ventilation.

5. Spray copper conductive spray onto only one side of the discs.

a. Keep nozzle perpendicular to discs surfaces 4-6 inches away.

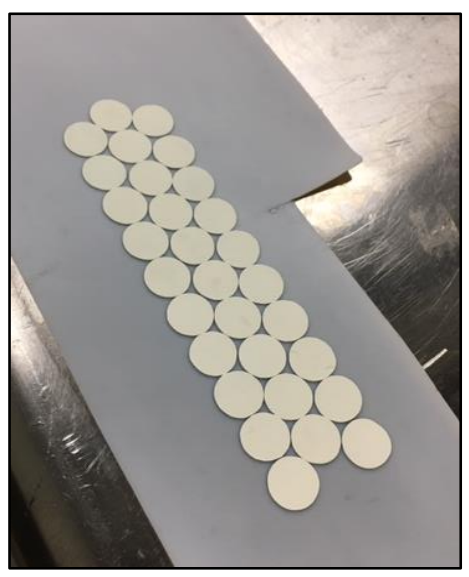

Figure A.9: Reusable Teflon mat

b. Spray an even coating across discs.

c. Let dry for 15 minutes.

6. Use scalpel to run along discs edges to remove excess electrode

a. This prevents shorting.

b. At a 45 degree angle, run scalpel along electroded surface to remove very minimal electrode at edge.

7. Clean with alcohol and proceed to poling procedure. 


\section{D.5: Corona Poling}

1. Place the corona poling setup on the hotplate.

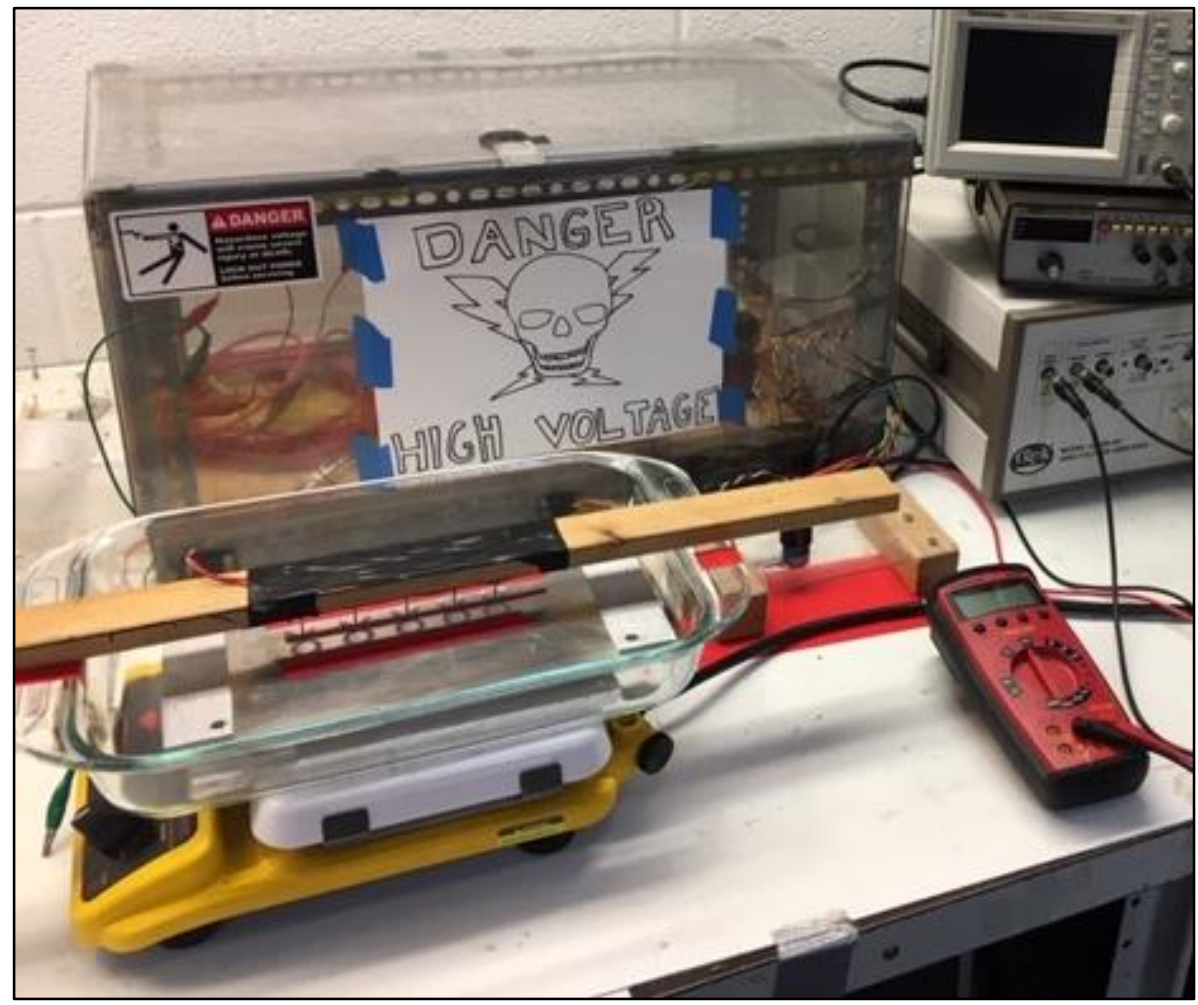

Figure A.10: Entire corona poling setup with 10 corona needles and a voltmeter to monitor voltage input.

2. Preheat hotplate to $100{ }^{\circ} \mathrm{C}$.

3. Place 10 composite discs electroded surface down.

a. One side of composite should remain unelectroded.

b. Align each disc under a corona needle.

c. The Needles should be 5-6.5 $\mathrm{mm}$ above disc surface. 


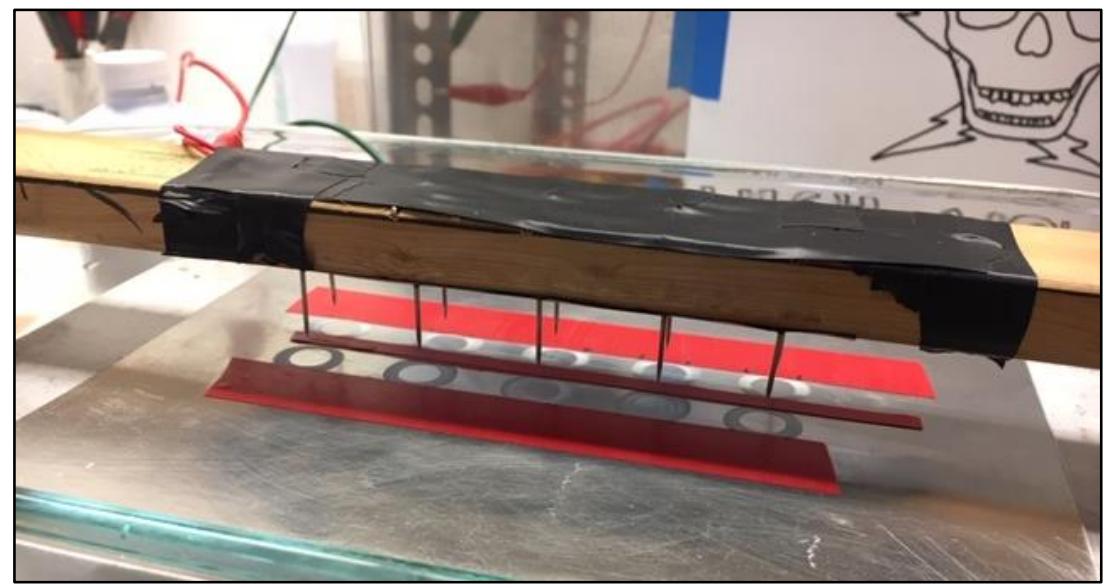

Figure A.11: Corona poling setup for through thickness poling of 10 composite discs.

4. Attach the voltmeter to the posts on the Faraday cage voltage control setup.

a. Set the voltmeter to measure DC voltage

5. With silicone glove and HV rubber glove on right hand, flip the faraday cage on and move delrin piece to lock circuit in the "on" position.

a. Keep left hand behind back

6. A steady voltage should appear on voltmeter of $\sim 10 \mathrm{mv}$.

a. If this is fluctuating, the poling setup is shorting

7. "Tap" or very slowly turn the potentiometer to increase the voltage.

a. At $0.4 \mathrm{~V}$ on the voltmeter (4000 V poling) the air should ionize

i. This should be an audible "hissing" sound

8. Increase the voltage until $0.6 \mathrm{~V}(6000 \mathrm{~V}$ poling $)$ is achieved

a. This is $15 \mathrm{kV} / \mathrm{mm}$ for $0.4 \mathrm{~mm}$ thick discs.

9. Hold the poling voltage for 30 minutes at $100{ }^{\circ} \mathrm{C}$

10. Turn off hotplate and maintain poling for another 30 minutes.

11. With the HV rubber glove, slowly turn potentiometer back to the steady $10 \mathrm{mV}$. 
12. Then, return the delrin lock to its initial position

a. Switch should return to off position.

13. Do not touch system until voltmeter settles to 0 Volts.

14. Remove from poling setup and store with electrodes short circuited for 24 hours.

a. Removes tapped charges in composite

15. Now, perform procedure D. 3 to place the second electrode.

a. Once discs are fully electroded and cleaned, proceed with CLACS manufacturing procedures written by Krech et al.

i. These can be found in Kyle Coates' Thesis 


\section{D.5: Half Rings}

The first procedure is used to manufacture the silicone mold used to shape, cure, and apply DEP to particle composites. The procedure steps and figures are given below.

1. 3D-print the desired negative for the composite shape that is desired.

a. For this study, a custom half-ring shape was designed for an IM nail.

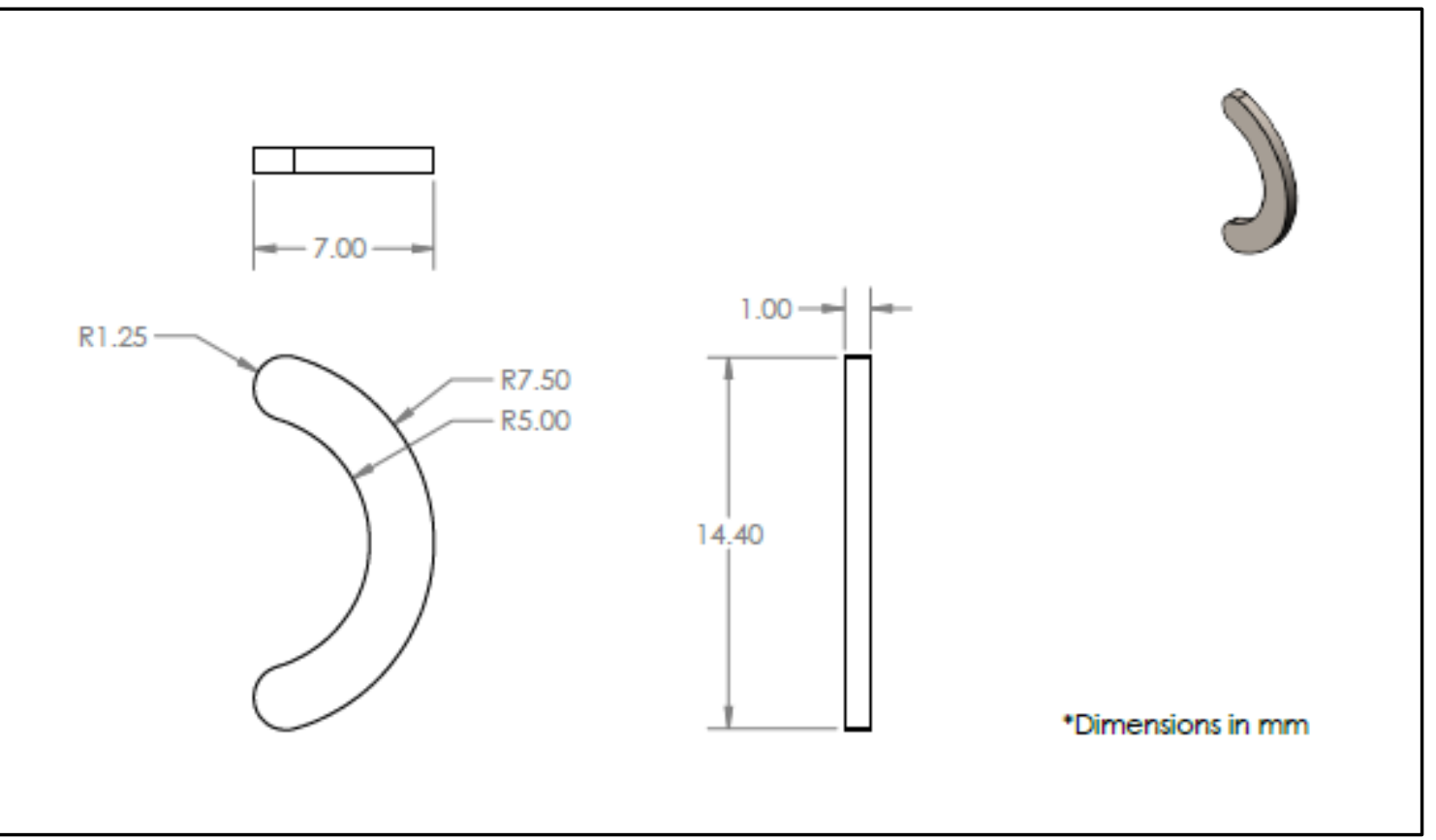

Figure A.12 - Half-ring negative (SolidWorks) 3D-printed for silicone mode.

2. Use double sided tape or super glue to adhere the negatives to a flat piece of delrin. Use hot glue to surround the negatives to form the shape of the mold.

3. Using medium cure silicone (Smooth-On, Macungie, PA), weight the needed mass of part A. For a 1-1 ratio, this would be equal to half of total mass needed. Then add equal part of B. Separate wood tongue depressors should be used to scoop dispense the silicone.

4. Using a third wood tongue depressor, mix the two parts slowly for about 2 minutes going both directions. 
5. Using a cotton swab, apply silicone oil to the tops of your negatives.

6. Then using the tongue depressor or wood end of cotton swab, transfer the mixed silicone to the mold, avoiding the tops of the negatives. Move from the outer edges in, and then just use the wood end of the cotton swab to spread out the silicone.

7. Let it settle, and use the flat end of the swab to scrape silicone off the top of the negatives and wipe with a paper towel. Continue to scrape and remove very small amounts from the surface of the negatives until the silicone remains off the top surface and is level with the top surface.

8. Let sit for 4-6 hours to cure with the top surface exposed (do not cover).

9. To remove, use a scalpel along the edges and peel away slowly. The goal is to leave the negatives adhered to the delrin so the setup can be easily used again.

10. This custom mold can now be used in Procedures D.1 through D.4 to produce custom shaped rings.

a. These shapes were a concept design for use in an intramedullary nail, which is discussed more in Appendix C.2. 
Appendix E: Additional Tables, Figures

\section{E.1 Additional Tables}

Table A.1: Average maximum power for all frequencies.

\begin{tabular}{|c|c|c|c|c|}
\hline & \multicolumn{4}{|c|}{ Average Maximum Power $(\boldsymbol{\mu W})$} \\
\hline Composite configuration & $\mathbf{1 ~ H z}$ & $\mathbf{2 ~ H z}$ & $\mathbf{3 ~ H z}$ & $\mathbf{5 ~ H z}$ \\
\hline random $0.0 \mathrm{~mm}$ & $22 \pm 4$ & $44 \pm 8$ & $66 \pm 12.3$ & $105 \pm 19$ \\
\hline random $0.8 \mathrm{~mm}$ & $27 \pm 10$ & $53 \pm 19$ & $78 \pm 29$ & $125 \pm 44$ \\
\hline structured $0.0 \mathrm{~mm}$ & $20 \pm 5$ & $39 \pm 10$ & $57 \pm 15$ & $91 \pm 23$ \\
\hline structured $0.8 \mathrm{~mm}$ & $32 \pm 3$ & $64 \pm 7$ & $94 \pm 9$ & $149 \pm 15$ \\
\hline
\end{tabular}

\section{E.2 Additional Figures}

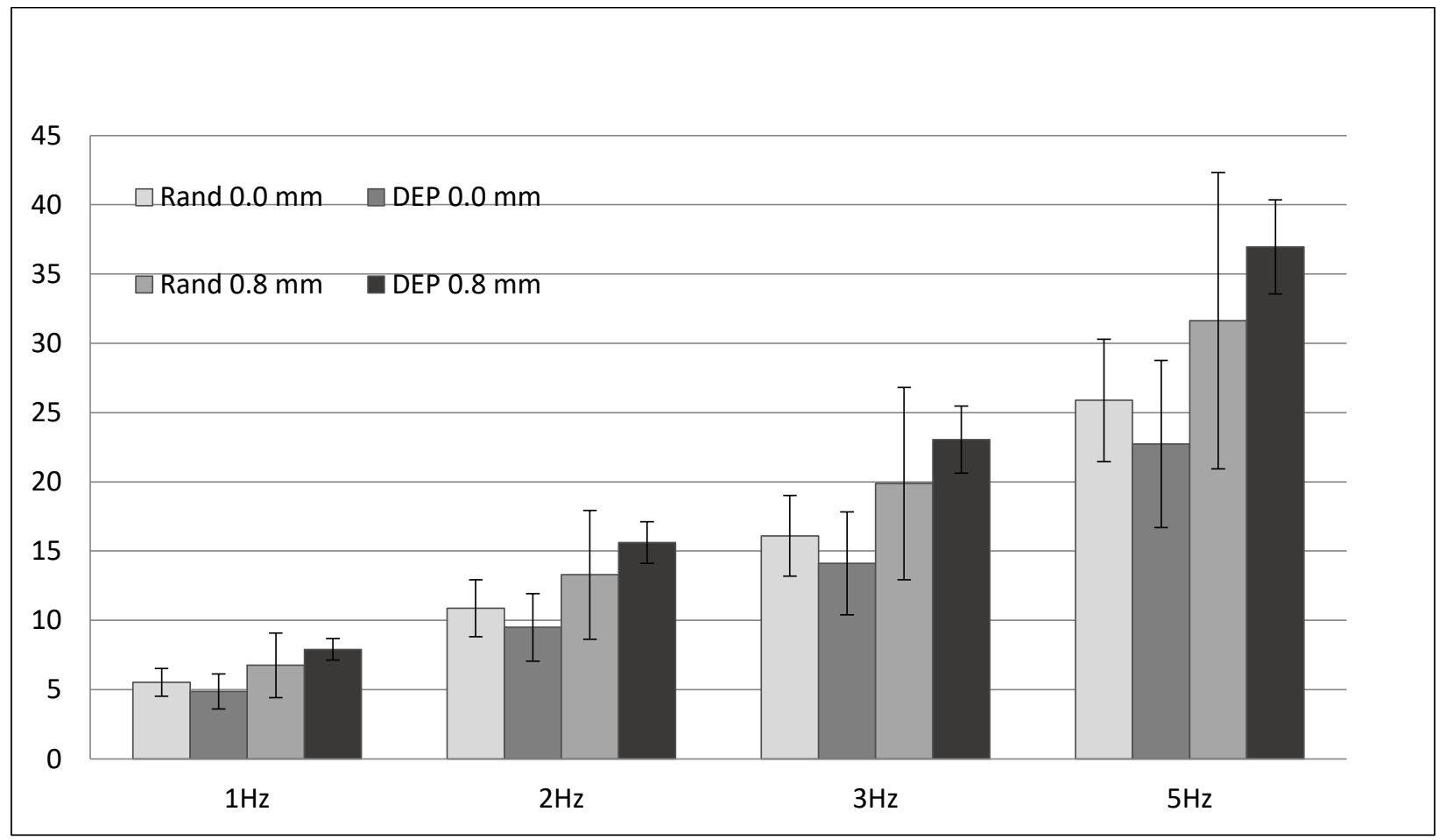

Figure A.13: Max power produced vs. frequency at $500 \mathrm{~N}$ and at the resistance for max power at each frequency $(167 \mathrm{M} \Omega, 77 \mathrm{M} \Omega, 57 \mathrm{M} \Omega, 40.5 \mathrm{M} \Omega$ respectively). 


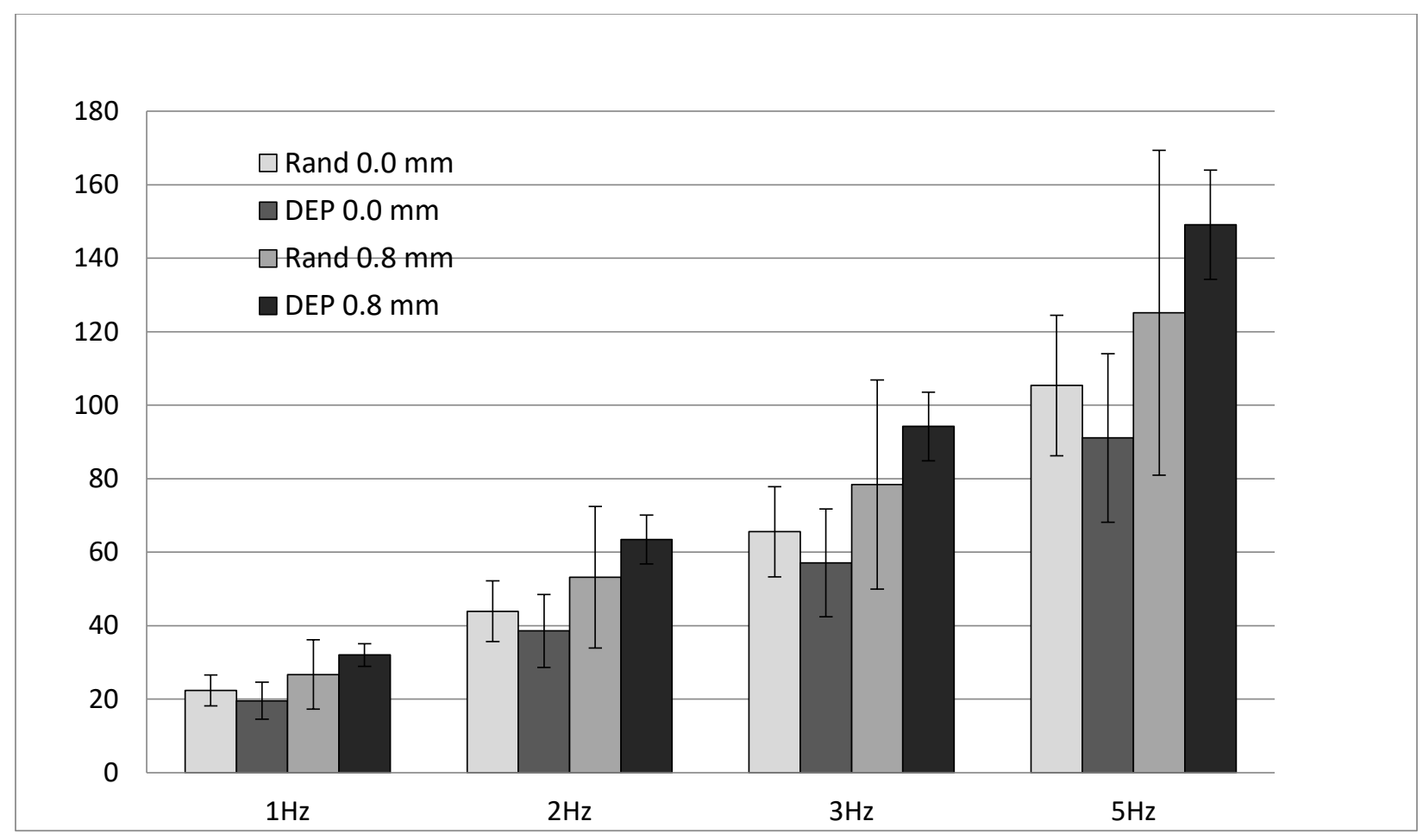

Figure A.14: Max power produced vs. frequency at $1000 \mathrm{~N}$ and at the resistance for max power at each frequency (167 $\mathrm{M} \Omega, 77 \mathrm{M} \Omega, 57 \mathrm{M} \Omega, 40.5 \mathrm{M} \Omega$ respectively).

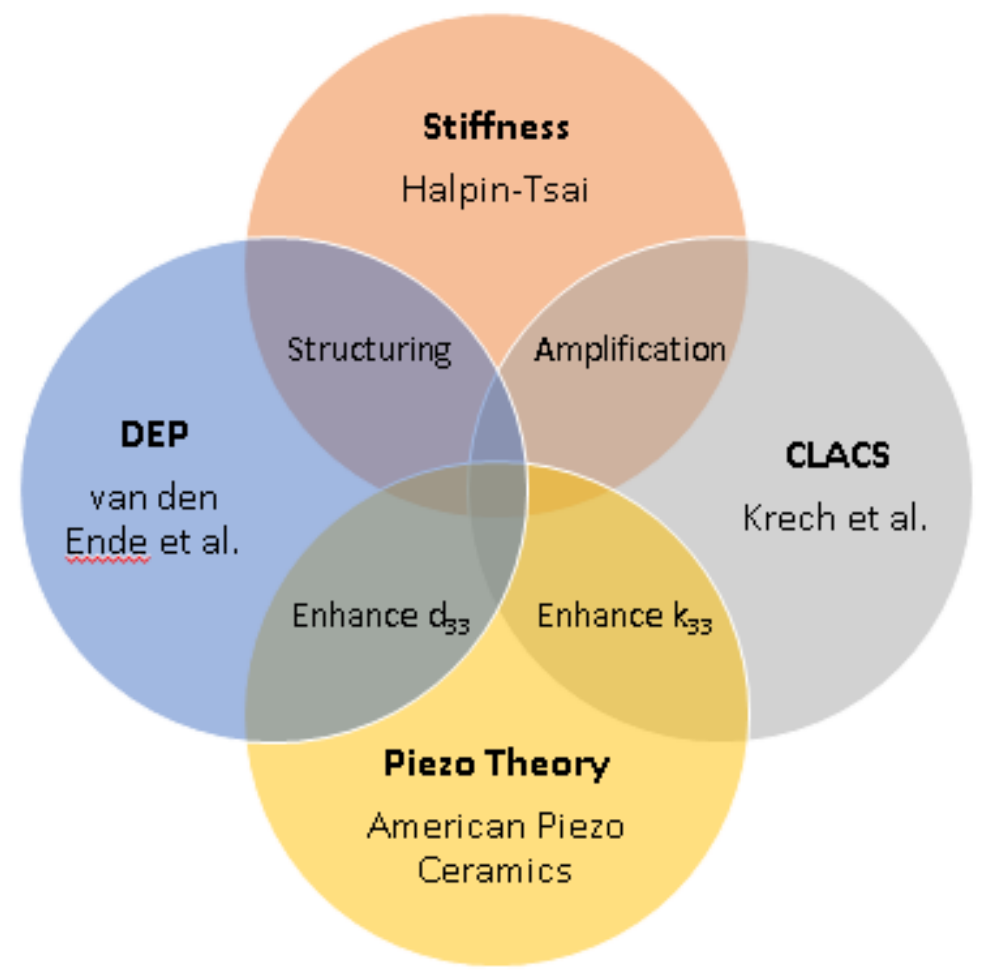

Figure A.15: Venn diagram to capture the development of the theoretical model. 


\section{E.3 Additional Information}

One potential setback to piezoelectric fracture fixation implants is the period of time where the patient must not bear weight on the fractured limb, since piezoelectric materials only produce current in sync with mechanical loading ${ }^{7}$. If patients receive dynamic intramedullary (IM) nailing, full weight bearing is encouraged as soon as inflammation subsides or within 4 weeks from surgery ${ }^{61}$. However, with static IM nailing, full weight bearing is not recommended until first signs of bone union are observed or until about 10 weeks ${ }^{62}$. According to a study by Arazi et al., weight bearing can also start within 2-4 weeks for static IM nailing ${ }^{63}$. To combat the lack of piezoelectric stimulation from mechanical loading within the first 4 weeks, ultrasound can be utilized to mechanically load PZT in medical implants ${ }^{64}$. Coates et al. demonstrated power production of PZT CLACS by means of ultrasound stimulation and displayed an increase in power production as compliant layer thickness increased ${ }^{54}$. This method of PZT stimulation can significantly help to further increase the rate of healing for fractures during within the first weeks. 